\title{
Mass-Conserving Method of Characteristics for Streamflow Modeling
}

United States

Geological Survey

Water-Supply

Paper 2369 


\section{AVAILABILITY OF BOOKS AND MAPS OF THE U.S. GEOLOGICAL SURVEY}

Instructions on ordering publications of the U.S. Geological Survey, along with the last offerings, are given in the current-year issues of the monthly catalog "New Publications of the U.S. Geological Survey." Prices of available U.S. Geological Survey publications released prior to the current year are listed in the most recent annual "Price and Availability List." Publications that are listed in various U.S. Geological Survey catalogs (see back inside cover) but not listed in the most recent annual "Price and Availability List" are no longer available.

Prices of reports released to the open files are given in the listing "U.S. Geological Survey Open-File Reports," updated monthly, which is for sale in microfiche from the U.S. Geological Survey Books and Open-File Reports Sales, Box 25425, Denver, CO 80225.

Order U.S. Geological Survey publications by mail or over the counter from the offices given below.

\section{BY MAIL}

\section{Books}

Professional Papers, Bulletins, Water-Supply Papers, Techniques of Water-Resources Investigations, Circulars, publications of general interest (such as leaflets, pamphlets, booklets), single copics of periodicals (Earthquakes \& Volcanoes, Preliminary Determination of Epicenters), and some miscellaneous reports, including some of the foregoing series that have gone out of print at the Superintendent of Documents, are obtainable by mail from

\section{U.S. Geological Survey, Books and Open-File Report Sales Box 25425 \\ Denver, CO 80225}

Subscriptions to periodicals (Earthquakes \& Volcanoes and Preliminary Determination of Epicenters) can be obtained ONLY from

\section{Superintendent of Documents \\ U.S. Government Printing Office Washington, DC 20402}

(Check or money order must be payable to Superintendent of Documents.)

\section{Maps}

For maps, address mail order to

$$
\begin{gathered}
\text { U.S. Geological Survey, Map Sales } \\
\text { Box 25286 } \\
\text { Denver, CO } 80225
\end{gathered}
$$

Residents of Alaska may order maps from

$$
\begin{aligned}
& \text { U.S. Geological Survey, Map Sales } \\
& 101 \text { Twelfth Ave., Box } 12 \\
& \text { Fairbanks, AK } 99701
\end{aligned}
$$

\section{OVER THE COUNTER}

\section{Books}

Books of the U.S. Geological Survey are available over the counter at the following U.S. Geological Survey offices, all of which are authorized agents of the Superintendent of Documents.

- ANCHORAGE, Alaska-4230 University Dr., Rm. 101

- ANCHORAGE, Alaska-605 West 4th Ave., Rm G-84

- DENVER, Colorado-Federal Bldg., Rm. 169, 1961 Stout St.

- LAKEWOOD, Colorado-Federal Center, Bldg. 810

- MENLO PARK, California-Bldg. 3, Rm. 3128, 345 Middlefield Rd.

- RESTON, Virginia-National Center, Rm. 1C402, 12201 Sunrise Valley Dr.

- SALT LAKE CITY, Utah-Federal Bldg., Rm. 8105, 125 South State St.

- SAN FRANCISCO, California-Customhouse, Rm. 504, 555 Battery St.

- SPOKANE, Washington-U.S. Courthouse, Rm. 678, West 920 Riverside Ave.

- WASHINGTON, D.C.-U.S. Department of the Interior Bldg., Rm. 2650, 1849 C St., NW.

Maps

Maps may be purchased over the counter at the U.S. Geological Survey offices where books are sold (all addresses in above list) and at the following Geological Survey offices:

- ROLLA, Missouri-1400 Independence Rd.

- FAIRBANKS, Alaska-New Federal Building, 101 Twelfth Ave. 
Mass-Conserving

Method of Characteristics

for Streamflow Modeling

By WILLIAM G. SIKONIA

U.S. GEOLOGICAL SURVEY WATER-SUPPLY PAPER 2369 


\title{
U.S. DEPARTMENT OF THE INTERIOR
}

MANUEL LUJAN, JR., Secretary

\author{
U.S. GEOLOGICAL SURVEY
}

Dallas L. Peck, Director

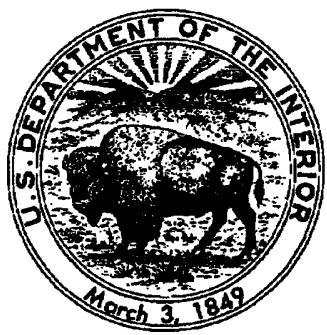

Any use of trade, product, or firm names in this publication is for descriptive purposes only and does not imply endorsement by the U.S. Government

UNITED STATES GOVERNMENT PRINTING OFFICE: 1992

For sale by the

Books and Open-File Reports Section

U.S. Geological Survey

Federal Center

Box 25425

Denver, CO 80225

\section{Library of Congress Cataloging-in-Publication Data}

Sikonia, W.G. (William G.)

Mass-conserving method of characteristics for streamflow modeling / by William G. Sikonia.

p. cm.-(U.S. Geological Survey water-supply paper ; 2369)

Includes bibliographical references.

Supt. of Docs. no.: I 19.3:W2369

1. Streamflow-Mathematical models. I. Title. II. Series. GB1207.S53 1992

$551.48^{\prime} 3^{\prime} 015118-\mathrm{dc} 20$ 


\section{CONTENTS}

Abstract 1

Introduction $\mathbf{1}$

Background 1

Purpose and scope 1

Description of the method of characteristics applied to streamflow modeling 2

Finite element formulation $\mathbf{3}$

Characteristics 8

Addition of continuity equation for mass conservation

Description of the equations for numerical solution 10

System of equations for the finite element solution 10

General form of partial derivatives for equations along characteristics

Partial derivatives with characteristic path fixed

Partial derivatives due to translation of the characteristic path

Partial derivatives for the continuity equation

19

Interpolation of channel parameters

19

Steep-slope factor 27

Model demonstrations and verifications

Example $1 \quad 28$

Example $2 \quad 35$

Future extension $\quad 38$

Summary and conclusions

References 42

Index of variable definitions, interpolations, and derivatives

\section{FIGURES}

1-4. Diagrams showing:

1. Characteristic paths 2

2. Finite element geometry 4

3. Approximation of characteristic paths 9

4. Variation of characteristic paths $\mathbf{1 2}$

5. Thalweg profiles for the examples 29

6. Data cross sections of the test river channel 31

7. Input discharge hydrographs for the examples 34

8-11. Computed water-depth profiles:

8. Example 136

9. Example 238

10. Effect of restriction of neighborhoods in time $\mathbf{4 0}$

11. Effect of restriction of neighborhoods in space 41

\section{TABLE}

1. Cross section geometry of the test river channel 
As an aid to locating expressions within the paper, the section "Index of Variable Definitions, Interpolations, and Derivatives" has also been provided at the end of the paper.

0 ............ Null vector

A .......... Cross-sectional area

$A^{*} \ldots . . . . . .=A$, but with distance $x$ along the river and depth $h$ as explicit independent variables

$a_{k} \ldots . . . . . . . . R e p r e s e n t s$ either $a_{k}^{Q}$ or $a_{k}^{h}$

$a_{j}^{h}$............ Nodal value of depth at one of four corner nodes or two midside nodes

$a^{h}$........... Column vector of nodal water depths

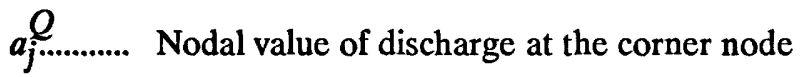

${ }_{a} Q \ldots$........ Column vector of nodal discharges

a ........... Column vector of nodal discharges $a$ nodal water depths a ${ }^{h}$

$B$......... Stream width at the water surface

$B^{*} \ldots . . . . .=B$, but with stream distance $x$ and water depth $h$ as explicit independent variables

c ........... $=\sqrt{g A / B}$, critical speed

$F^{\prime}$.......... Derivative matrix

$F_{c}$........ Integral of the continuity equation over a space-time element

$\hat{F}_{c} \ldots \ldots . . .=F_{c}$, but with nodal discharges a $Q$ and nodal water depths $\mathbf{a}^{h}$ as explicit independent variables

$F_{g^{ \pm} \ldots . . . .}$ Differential expression that for an exact solution is equal to zero along the positive $(+)$ or negative $(-)$ characteristic

$\hat{F}_{g^{ \pm}} \ldots \ldots . . .=F_{g^{ \pm}}$, but with nodal discharges a $Q$ and nodal water depths $\mathbf{a}^{h}$ as explicit independent variables

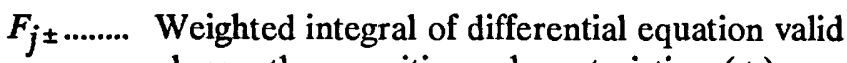
along the positive characteristic $(t)$ or negative characteristic $(-)$ through node $j$
$\hat{F}_{j \pm \ldots \ldots . . .}=F_{j \pm}$, but with nodal discharges a $Q$ and nodal water depths $\mathbf{a}^{h}$ as explicit independent variables

g ............ Acceleration of gravity

$G$......... Right side of momentum equation

h ........... Water depth

$\hat{h}$............ Integration parameter corresponding to depth $h$

$h_{k} \ldots \ldots . . . .$. Water depth corresponding to elevation $z_{k}$ at which data are specified

$\dot{h}_{ \pm}$.......... Total time derivative of depth $h$ along the positive characteristic $(+)$ or negative characteristic (-)

$i$........... As superscript, indicates intercept with the boundary of the neighborhood of restriction

$I_{ \pm} \ldots . . . . . . .$. Integrand along the positive $(+)$ or negative $(-)$ characteristic

J ........... Jacobian matrix

$|\mathbf{J}|$....... Determinant of the Jacobian matrix

$\mathrm{J}^{-1}$........ Inverse of the Jacobian matrix

$\ell$........... Index of node along the approximation of the characteristic path

$m$......... Number of subintervals into which the local time interval $\left[\eta_{\ell-1}, \eta_{\ell}\right]$ is divided; also, the number of elevation nodes within a data cross section

$n$.......... Manning's roughness coefficient; also, the number of data cross sections

$n^{*}$.......... $=n$, but with stream distance $x$ and water depth $h$ as explicit independent variables

$n_{J}$.......... Manning's coefficient using Jarrett's formula

$\tilde{n}$........... Slope-corrected Manning's coefficient 
$N$.

Number of elements

$N_{j} \ldots . . . . . . . \quad$ Represents either $N_{j} Q_{\text {or }} N_{j}^{h}$

$N_{j}^{h}$.......... Shape function that is linear in time $t$ and quadratic in space $x$

$N_{j}=\ldots . . . . .$. Shape function that is linear in both time $t$ and space $x$

$P$.......... Wetted perimeter

$P^{*} \ldots . . . . . .=P$, but with stream distance $x$ and water depth $h$ as explicit independent variables

$q$.......... Lateral inflow (discharge) per unit distance along the river

Q .......... Discharge

$\dot{Q}_{ \pm} \ldots . . . . .$. Total time derivative of discharge $Q$ along the positive characteristic $(+)$ or negative characteristic (-)

$R$.......... $=A / P$, hydraulic radius

$R_{0} \ldots . . . . . .=$ Reference hydraulic radius; 7 feet in this report

$S$.......... Slope

$S_{0} \ldots \ldots . . . . .=$ Reference slope, 0.002 in this report

$S_{f}$.......... Friction slope

$S_{f_{0}} \ldots . . .$. Friction slope given by Manning's expression, unmodified for steep slopes

$S_{w} \ldots . . . .$. Water surface slope

$S_{e}$......... Exponent in the steep-slope factor $\Phi$

$S_{\text {fac }}$..... Coefficient in the steep-slope factor $\Phi$

$S_{\min }$.... Quantity related to water surface slope, used in the steep-slope factor $\Phi$

t ............. Time

$\Delta t$.......... Increment of time $t$

$t_{j}$........... Nodal value of time $t$

$T$.......... As superscript, indicates transpose of a matrix

$u$........... $=Q / A$, average stream velocity $u_{q}$......... Velocity component of lateral inflow in the longitudinal direction $x$

$v$.......... As superscript, indicates the vertex of the space-time curves of dependence

$W_{j \pm} \ldots . .$. Weighting function for integration along the positive characteristic $(+)$ or negative characteristic $(-)$ passing through node $j$

$x$............ Longitudinal distance along the river

$\Delta x \ldots . . . .$. Increment of spatial distance $x$

$x_{j}$.......... Computational nodal value of river distance $x$, or stream coordinate at which data are specified

$\dot{x}_{ \pm} \ldots \ldots . . . . .=u \pm \sqrt{g A / B}$, total time derivative of river distance $x$ along the positive characteristic $(+)$ or negative characteristic $(-)$

$\dot{x}_{\mp} \ldots . . . . . . .=u \mp \sqrt{g A / B}$, total time derivative of river distance $x$ along the opposite characteristic from $x_{ \pm}$when using upper or lower signs

$z$............ Elevation

$\Delta z$......... Increment of elevation

$z_{b}$........... Channel thalweg (channel bottom) elevation

$z_{k}$.......... Elevation within a data cross section at which data are specified

$\alpha, \quad \beta \quad \ldots$ Expressions used in approximating $\left.\frac{\partial \xi}{\partial a_{k}}\right|_{\ell}$

$\Gamma$........... Path of a characteristic

$\delta$........... Indicates variation of the following variable

$\in \ldots . . . . . .$. Read "is an element of "

$\zeta$........... Local depth coordinate

$\hat{\zeta}$........... Integration parameter corresponding to local depth $\zeta$

$\eta$........... "Time" coordinate of local element

$\Delta \eta \quad$......... Increment of local time coordinate $\eta$

$\hat{\eta}$........... Integration parameter for local time coordinate $\eta$ 
$\eta_{\ell}$......... Local time coordinate of a node along the approximation of the characteristic path

$\Delta \eta_{\ell} \ldots . . . .=\eta_{\ell}-\eta_{\ell-1}$, local time increment along the approximation of the characteristic path

$\eta_{\ell, i}$...... ith auxiliary node within the local time interval $\left[\eta_{\ell-1}, \eta_{\ell}\right]$

$\eta_{j \pm}^{i}$...... Local time coordinate of the "intercept" with the neighborhood of restriction of the positive characteristic $(+)$ or negative characteristic $(-)$ that passes through node $j$

$\eta \nu$......... Value of the local time variable $\eta$ at the vertex of the characteristic

$\eta_{j \neq}^{\nu}$...... Local time coordinate of the "vertex" at node $j$ of the positive characteristic $(+)$ or negative characteristic (-)

$\theta$.......... Parameter used in setting the time extent of the neighborhood of restriction

$\xi$.......... "Space" coordinate of local element

$\Delta \xi$........ Increment of local space coordinate $\xi$

$\xi_{\ell}$......... Local space coordinate of a node along the approximation of the characteristic path

$\xi^{v}$.......... Value of the local space variable $\xi$ at the vertex of the characteristic

$\dot{\xi}_{ \pm} \ldots . . . . .$. Total $\eta$ derivative along the characteristic path in local coordinates
$\Xi$........... Local "river distance" coordinate for channel parameter interpolation

$\rho, \sigma, \lambda, \nu$.. Subscript increments in the definition of the approximation of derivatives for Hermite interpolation

$\Sigma$........... Summation sign

$\Phi$........... Steep-slope factor for friction slope $S_{f}$

$\phi_{0 m}, \phi_{0 p}, \phi_{1 m}, \phi_{1 p} .$. Basis functions for Hermitian interpolation in local river distance $\Xi$

$\chi$.......... Parameter used in setting the space extent of the neighborhood of restriction

$\psi_{0 m}, \psi_{0 p}, \psi_{1 m}, \psi_{1 p} .$. Basis functions for Hermitian interpolation in local depth $\zeta$

Notation above a variable indicating average value

| .......... Notation indicating evaluation of the expression to the left at a particular point

$\int \ldots . . . . . . . .$. Integral sign

a ........... Partial derivative sign

$\approx$............ Approximate equality sign

Read "is defined as"

\section{METRIC CONVERSION FACTORS}

For the convenience of readers, the inch-pound units used in this report may be converted to metric units by using the following factors:

\begin{tabular}{lll}
\hline Multiply inch-pound units & \multicolumn{1}{c}{ By } & \multicolumn{1}{c}{ To obtain metric units } \\
\hline foot $(\mathrm{ft})$ & 0.3048 & meter $(\mathrm{m})$ \\
cubic foot $\left(\mathrm{ft}^{3}\right)$ & 28.32 & liter $(\mathrm{L})$ \\
& 0.02832 & cubic meter $\left(\mathrm{m}^{3}\right)$ \\
cubic foot per second $\left(\mathrm{ft}^{3} / \mathrm{s}\right)$ & 28.32 & liter per second $(\mathrm{L} / \mathrm{s})$ \\
& 0.02832 & cubic meter per second $\left(\mathrm{m}^{3} / \mathrm{s}\right)$ \\
\hline
\end{tabular}

In addition, Manning's expression for friction slope, equation 93, may be expressed by

$$
S_{f_{0}}=\frac{n^{2}|Q| Q P^{4 / 3}}{A^{10 / 3}},
$$

where discharge $Q$ has units cubic meters per second, area $A$ is specified in square meters, and wetted perimeter $P$ is given in meters. 


\title{
Mass-Conserving Method of Characteristics for Streamflow Modeling
}

\author{
By William G. Sikonia
}

\begin{abstract}
A robust numerical model is presented for the computation of unsteady streamflow on steep river slopes. The one-dimensional model uses the method of characteristics on a specified space-time grid to solve the Saint-Venant equations. An additional continuity equation requirement on each space-time element provides greatly improved conservation of mass over traditional implementations of the method of characteristics on a fixed grid. The space-time geometry of the problem is described in a finite element setting. Hermite interpolation of channel parameters is used to avoid numerical difficulties that may occur with steep slopes due to discontinuities in the derivatives of data such as channel top width. Manning's equation for friction slope can be modified by a factor to make the slope more appropriate for steep rivers. The standard Manning's friction slope can also be used, if preferred. The computer model is not restricted to steep slopes, and applies as well to gently sloping streams. Two numerical examples support the mathematical approach and computational algorithm.
\end{abstract}

\section{INTRODUCTION}

\section{Background}

The implicit method of characteristics is an appealing method for solving hyperbolic differential equations. This method has been applied in the context of hydraulics to solve the Saint-Venant equations, which describe onedimensional, unsteady, open-channel flow (Edenhofer and Schmitz, 1981). In the method, characteristics are projected backward only within a given element on a fixed space-time grid (fig. 1), and are never extrapolated beyond the boundaries of that element. For the space-time element 1-2-3-4 in figure 1, characteristics either intersect one of the vertical boundaries (1-4 or 2-3), as characteristic $a b$ does, or intersect the base (1-2) of the element, as characteristic de does. (The mathematical construction of characteristics will be given in succeeding sections for readers unfamiliar with the method.) In some instances, however, the method has resulted in unacceptable nonconservation of mass (Wylie, 1980). Wylie referred to nonconservation in the case in which a characteristic, such as $d e$ in figure 1 , intersects the base of the space-time element. The situation was further compounded because the method seemed to perform well for some applications but produced serious inaccuracies for others. In the same paper, Wylie described a method of "time-line" interpolations to improve mass conservation; more discussion of the "time-line" method, and further insight into the problem was provided later by Goldberg and Wylie (1983). The "time-line" method involves projecting the characteristics backward in time until a vertical boundary of the space-time grid is intersected, even if this means projecting through the base of the current space-time element into elements at preceding times, such as extending characteristic de in figure 1 backwards in time along ef. Note that if a characteristic (such as $a b$ in figure 1) intersects the vertical boundary within the originating space-time element, the "time-line" and implicit methods of characteristics are identical. See also Lai (1988) for a composite algorithm for the method of characteristics that includes these approaches, as well as another variant involving extension of a characteristic such as $a b$ in figure 1 outward in distance along $b c$.

\section{Purpose and Scope}

This paper presents a solution method that will preserve the desirable features of the method of characteristics, but will also conserve mass to a satisfactory approximation. The method differs from the other approaches just mentioned. In particular, this method does not involve extrapolation of the characteristic beyond the originating element.

The paper is organized as follows. The mathematical basis of the method of characteristics, as applied to the Saint-Venant equations for streamflow, is presented first. The finite element setting that is used for the numerical 


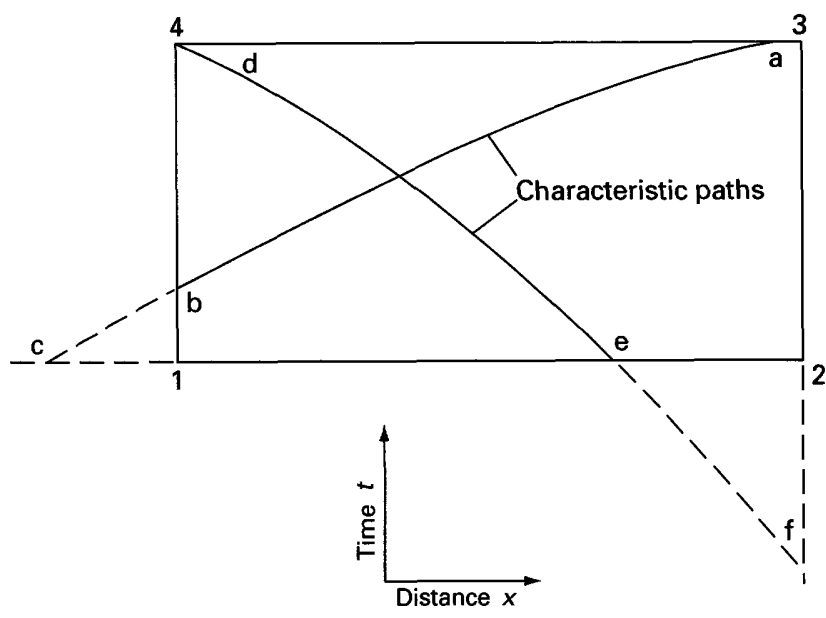

Figure 1. Characteristic paths projected within and beyond element 1-2-3-4.

procedure is described next; the unknown discharge $Q$ and water depth $h$ are approximated by shape function expansions on space-time finite elements. A numerical procedure for approximating the characteristic paths is presented; then the continuity equation is included as part of the procedure to provide mass conservation over the space-time elements. The global system of equations that is solved within the method is described next. The underlying nonlinear global system is approximated, using Newton-Raphson iteration, by a linear global system. The nonlinear and linear global systems of equations are simply expressed in symbolic, condensed form in vector and matrix notation. The symbolic form of the approximating linear system is expanded by providing partial derivatives needed for its expression. Next, independent Hermitian interpolation is used to describe channel geometry. Description of the model is completed with a discussion of a factor that can be applied to Manning's coefficient to account for steep slopes. Finally, two comparisons of the model of this paper with existing models are given. In the first example, the model is compared on a steep river with a step-backwater, steadystate model; in the second example it is compared with an existing dynamic model.

\section{DESCRIPTION OF THE METHOD OF CHARACTERISTICS APPLIED TO STREAMFLOW MODELING}

One-dimensional open-channel flow is described by the Saint-Venant equations, consisting of the continuity, or mass conservation, equation

$$
B \frac{\partial h}{\partial t}+\frac{\partial Q}{\partial x}=q
$$

and the momentum equation

$$
\frac{\partial Q}{\partial t}+\frac{\partial}{\partial \mathrm{x}}\left(\frac{Q^{2}}{A}\right)+g A \frac{\partial h}{\partial \mathrm{x}}+g A\left(s_{f}+\frac{\partial z_{b}}{\partial \mathrm{x}}\right)-u_{q} q=0 \text {. }
$$

In equations 1 and $2, B$ is stream width at the water surface, $h$ is depth, $t$ is time, $Q$ is discharge, $x$ is longitudinal distance along the river, $q$ is lateral inflow (discharge) per unit $x$ distance, $A$ is cross-sectional area, $g$ is acceleration of gravity, $z_{b}$ is the thalweg (channel bottom) elevation, $u_{q}$ is the downstream velocity component of lateral inflow, and $S_{f}$ is the friction slope.

Let

$$
A(x, t)=A^{*}(x, h(x, t))
$$

then

$$
\frac{\partial A}{\partial x}=\frac{\partial A^{*}}{\partial x}+\frac{\partial A^{*}}{\partial h} \frac{\partial h}{\partial x}=\frac{\partial A^{*}}{\partial x}+B \frac{\partial h}{\partial x}
$$

Expanding the second term of equation 2, substituting equation 4 , and rearranging terms yields

$$
\begin{aligned}
\frac{\partial Q}{\partial t} & +2 u \frac{\partial Q}{\partial x}+\left(-B u^{2}+g A\right) \frac{\partial h}{\partial x} \\
& =u^{2} \frac{\partial A^{*}}{\partial x}-g A\left(s_{f}+\frac{\partial z_{b}}{\partial x}\right)+u_{q} q \\
& =G .
\end{aligned}
$$

In equation $5, u=Q / A=$ average stream velocity. Characteristics are specified when the following system of equations is linearly dependent; this occurs when application of Cramer's rule of determinants results in the indeterminate form " $0 / 0$ ".

$$
\left[\begin{array}{cccc}
1 & 0 & 0 & B \\
2 u & 1 & \left(-B u^{2}+g A\right) & 0 \\
\dot{x} & 1 & 0 & 0 \\
0 & 0 & \dot{x} & 1
\end{array}\right]\left[\begin{array}{l}
Q_{\mathrm{x}} \\
Q_{t} \\
h_{\mathrm{x}} \\
h_{t}
\end{array}\right]=\left[\begin{array}{l}
q \\
G \\
\dot{Q} \\
\dot{h}
\end{array}\right] .
$$

In equation 6 , the dot notation indicates total time derivative. Setting the determinant of the matrix on the left side of equation 6 to zero (to provide the zero in the denominator of the " $0 / 0$ " relation) yields, after some alge- 
bra, the equations that specify the locations of the characteristics in the $x$ - $t$ plane:

$$
\dot{x}_{ \pm}=u \pm \sqrt{g A / B} \text { for upper, lower signs. }
$$

In equations 7 , the notation $\dot{x}_{ \pm}$denotes the total time derivative of $x$ along the positive characteristic (upper signs), or negative characteristic (lower signs). The extremely convenient notation \pm that will be used throughout the paper has a dual interpretation in mathematics. In singular usage, as in the phrase "equation $\dot{x}_{ \pm}=u \pm \sqrt{g A / B}$," the \pm is, in effect, notation for a general expression where a choice of one sign $(+)$ or the other $(-)$ can be made, much in the same way that an index $n$ on $(-1)^{n}$ can be thought of as a symbolic quantity. The plural interpretation of \pm , as in the phrase "equations $\dot{x}_{ \pm}=u \pm \sqrt{g A / B}$," refers to the set of equations formed by enumerating the possibilities, first with the upper sign $(+)$, and then with the lower sign $(-)$; this is similar to considering the set of numbers $(-1)^{n}$, for $n=1,2$. Whether the singular, symbolic usage or the plural enumeration of the set of possibilities is intended will depend on the context in which the \pm occurs. To emphasize that equations 7 mean the set of two characteristics we include the phrase "for upper, lower signs," which serves the same function as the phrase "for $n=1,2$ " in the example of the index. One further comment is necessary: In interpreting a set of equations such as equations 7 , one should consistently use the upper signs or the lower signs throughout. The reason for this caution is demonstrated below in equations 8 , which have mixed \pm and $\mp$ signs.

Replacing the third column of the matrix in equation 6 by the column vector on the right side of that equation, and setting the determinant of the resultant matrix to zero (to provide the zero in the numerator of the " $0 / 0$ " relation) yields ordinary differential equations that are satisfied along the characteristic curves of equations 7 :

$$
\begin{gathered}
\dot{Q}_{ \pm}-\dot{x}_{T} B \dot{h}_{ \pm}-q\left(u_{q}-\dot{x}_{\mp}\right)-u^{2} \frac{\partial A}{\partial x}+g A\left(s_{f}+\frac{\partial z_{b}}{\partial x}\right)=0 \\
\text { for upper, lower signs. }
\end{gathered}
$$

The equation associated with the upper signs in equations 8 is satisfied along the positive characteristic specified by the upper signs in equations 7. Similarly, the lower signs specify the negative characteristic (equations 7 ) and the equation satisfied along it (equations 8).

The notations $\dot{Q}_{ \pm}$and $\dot{h}_{ \pm}$denote total time derivatives of $Q$ and $h$ along the respective characteristics. Note that the slope of the opposite characteristic $\dot{x}_{\mp}$ appears in equations 8 , not the slope $\dot{x}_{ \pm}$of the characteristic itself:

$$
\dot{x}_{\mp}=u \mp \sqrt{g A / B} .
$$

\section{Finite Element Formulation}

Equations 8 are solved numerically along characteristics (equations 7) in a space-time finite element (12-3-4 in fig. 2). The directions 1-2 and 4-3 correspond to an increment $\Delta x$ of spatial distance along river coordinate $x$, and the directions 1-4 and 2-3 correspond to an increment of time $\Delta t$, from the "old" time at 1 or 2 , to the "new" time at 4 or 3.

Variables are defined within the finite element by a linear combination of shape functions having nodal values as coefficients. The discharge $Q$ has a bilinear approximation on nodal values at points $1,2,3$, and 4 ,

$$
Q=\sum_{j=1}^{4} N_{j}^{Q}(x, t) a_{j}^{Q}
$$

In equation 10 , each $N_{j}^{Q}(x, t)$ denotes a bilinear shape function over the space-time element that is unity at node $j$ and zero at the other three corner nodes. The notation $a_{j}^{Q}$ denotes the nodal value of discharge $Q$ at corner node $j$, and the summation is over the four corner nodes.

For the reason to be explained later in the section "Addition of Continuity Equation for Mass Conservation," the water depth $h$ is approximated linearly in time, but quadratically in distance. To this end, auxiliary nodes 5 and 6 are placed at the midpoints of the sides 1-2 and 3-4 (fig. 2). Water depth $h$ is then given by

$$
h=\sum_{j=1}^{6} N_{j}^{h}(x, t) a_{j}^{h} .
$$

In equation 11 , each $N_{j}^{h}(x, t)$ is a shape function that is linear in time and quadratic in space and is unity at node $j$ and zero at the other nodes. The summation index $j$ ranges over six nodes, namely the four corner nodes $1,2,3$, and 4, and two midside nodes 5 and 6 . The notation $a_{j}^{h}$ denotes the nodal value of depth $h$ at node $j$.

It is most convenient to work in a standard local element of fixed dimensions, and to define the basis functions on this local element. To this end, the variably sized space-time element 1-2-3-4, with sides $\Delta x$ and $\Delta t$, is mapped into the local element in independent variables $\xi$ and $\eta$ (fig. 2). The local element is square, with sides $\xi \in[-1,1]$ and $\eta \in[-1,1]$. (For simplicity, the corresponding nodes of the local and global elements have been identified by the same local node numbers; in practice, an additional set of global node numbers is needed to identify the multiple global elements.) Local variable $\eta$ corresponds to the time 
Global element

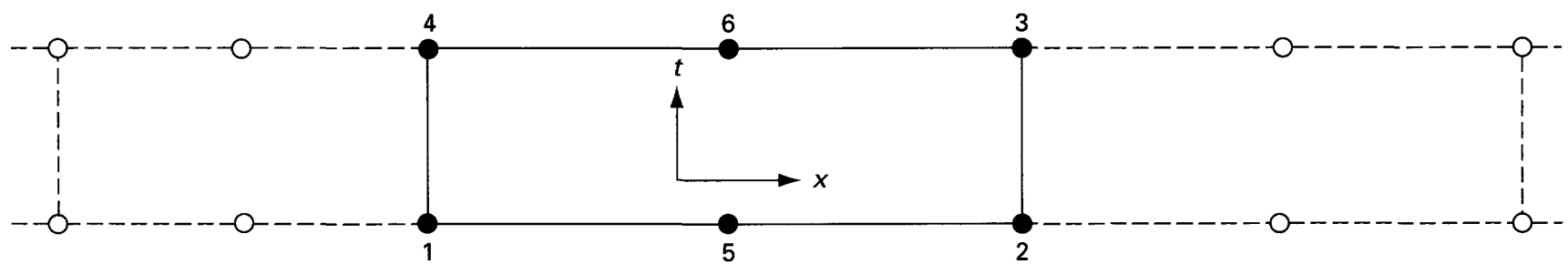

Local element

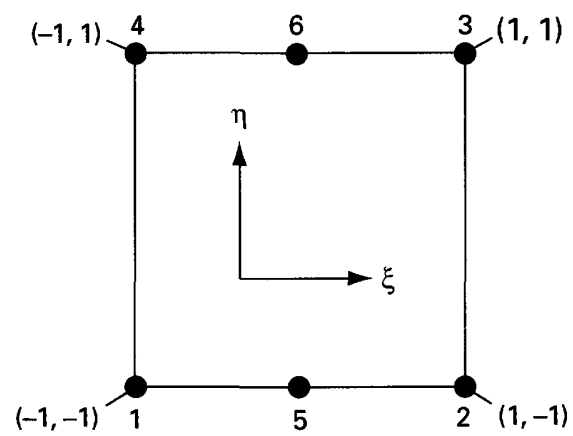

Figure 2. Finite element geometry.

variable $t$ in global coordinates, and $\xi$ corresponds to global space coordinate $x$. The transformation between global and local elements is defined by the same basis functions $N_{j}^{h}$ used for expanding the water depth $h$. The $N_{j}^{h}$ are defined on the local element as follows:

Corner nodes

$$
\begin{aligned}
& N_{1}^{h}=-\xi(1-\xi)(1-\eta) / 4 \\
& N_{2}^{h}=\xi(1+\xi)(1-\eta) / 4 \\
& N_{3}^{h}=\xi(1+\xi)(1+\eta) / 4 \\
& N_{4}^{h}=-\xi(1-\xi)(1+\eta) / 4 .
\end{aligned}
$$

Midside nodes

$$
\begin{aligned}
& N_{5}^{h}=\left(1-\xi^{2}\right)(1-\eta) / 2 \\
& N_{6}^{h}=\left(1-\xi^{2}\right)(1+\eta) / 2 .
\end{aligned}
$$

The billinear shape functions $N_{j}^{Q}$ are given on the local element by the following:
Corner nodes

$$
\begin{aligned}
& N_{1}^{Q}=(1-\xi)(1-\eta) / 4 \\
& N_{2}^{Q}=(1+\xi)(1-\eta) / 4 \\
& N_{3}^{Q}=(1+\xi)(1+\eta) / 4 \\
& N_{4}^{Q}=(1-\xi)(1+\eta) / 4 .
\end{aligned}
$$

The mapping from local to global coordinates is specified using the shape functions $N_{j}^{h}$, as follows:

$$
\begin{aligned}
& x(\xi, \eta)=\sum_{j=1}^{6} N_{j}^{h} x_{j} \\
& t(\xi, \eta)=\sum_{j=1}^{6} N_{j}^{h} t_{j}
\end{aligned}
$$

where the summation in $j$ is over the six corner and midside nodes, and $x_{j}$ and $t_{j}$ are the nodal values of river distance $x$ 
and time $t$ at the $j$ th node. Because we have chosen always to place the midside nodes exactly halfway between the corner nodes, the mapping defined by equations 22 and 23 actually reduces to a linear one. To prove the linearity, expand equation 22 to give

$$
\begin{aligned}
x= & \sum_{j=1}^{6} N_{j}^{h} x_{j} \\
= & -\frac{1}{4} \xi(1-\xi)(1-\eta) x_{1}+\frac{1}{4} \xi(1+\xi)(1-\eta) x_{2} \\
& +\frac{1}{2}\left(1-\xi^{2}\right)(1-\eta) \frac{1}{2}\left(x_{1}+x_{2}\right) \\
& +\frac{1}{4} \xi(1+\xi)(1+\eta) x_{3}-\frac{1}{4} \xi(1-\xi)(1+\eta) x_{4} \\
& +\frac{1}{2}\left(1-\xi^{2}\right)(1+\eta) \frac{1}{2}\left(x_{3}+x_{4}\right) \\
= & \frac{1}{4}(1-\xi)(1-\eta) x_{1}+\frac{1}{4}(1+\xi)(1-\eta) x_{2} \\
& +\frac{1}{4}(1+\xi)(1+\eta) x_{3}+\frac{1}{4}(1-\xi)(1+\eta) x_{4} \\
& =\sum_{j=1}^{4} N_{j} x_{j}
\end{aligned}
$$

Similarly, expanding equation 23 yields

$$
t=\sum_{j=1}^{4} N_{j}^{Q} t_{j}
$$

This completes the proof of linearity.

Derivatives associated with the mapping between local and global coordinates, equations 22 and 23 or 24 and 25 , are needed throughout processing. Partial derivatives of $x$ and $t$ with respect to $\xi$ and $\eta$ can be obtained formally from either pair of equations by substituting the appropriate derivatives from equations 12 through 17 , or equations 18 through 21 . Because of the rectangular geometry of the global element, however, the derivatives can be even more simply represented. Recognizing that $x_{1}=x_{4}$ and $x_{2}=x_{3}$, equation 24 yields

$$
\begin{aligned}
& x=\left[(1-\xi) x_{1}+(1+\xi) x_{2}\right] / 2 \\
& \frac{\partial x}{\partial \xi}=\frac{d x}{d \xi}=\left(x_{2}-x_{1}\right) / 2 \\
& \frac{\partial x}{\partial \eta}=0 .
\end{aligned}
$$

Similarly, recognizing that $t_{1}=t_{2}$ and $t_{3}=t_{4}$, we obtain from equation 25 that

$$
\begin{aligned}
& t=\left[(1-\eta) t_{1}+(1+\eta) t_{4}\right] / 2 \\
& \frac{\partial t}{\partial \eta}=\frac{d t}{d \eta}=\left(t_{4}-t_{1}\right) / 2 \\
& \frac{\partial t}{\partial \xi}=0 .
\end{aligned}
$$

The derivative of the mapping between local and global coordinates is given by the Jacobian matrix

$$
\mathrm{J}=\left[\begin{array}{ll}
\frac{\partial \mathrm{x}}{\partial \xi} & \frac{\partial \mathrm{x}}{\partial \eta} \\
\frac{\partial t}{\partial \xi} & \frac{\partial t}{\partial \eta}
\end{array}\right]
$$

Using the chain rule for differentiation yields

$$
\left[\begin{array}{ll}
\partial N_{j} & \frac{\partial N}{\partial \xi} \\
\partial \eta
\end{array}\right]=\left[\begin{array}{ll}
\partial N_{j} & \frac{\partial N}{\partial x} \\
\frac{\partial t}{\partial x}
\end{array}\right] \mathbf{J},
$$

where we use the same notation $N_{j}$ for the shape function as a function of local coordinates $(\xi, \eta)$ or global coordinates $(x, t)$, the distinction being made by context. $N_{j}$ represents either the $j$ th shape function $N_{j}^{Q}$ or $N_{j}^{h}$. Inversely to equation 33 , we have

$$
\left[\begin{array}{ll}
\partial N_{j} & \frac{\partial N}{\partial x} \\
\frac{\partial t}{\partial t}
\end{array}\right]=\left[\begin{array}{ll}
\partial N_{j} & \frac{\partial N}{\partial \xi} \\
\frac{\partial \eta}{\partial \eta}
\end{array}\right] \mathbf{J}^{-1},
$$

where the inverse Jacobian matrix $\mathbf{J}^{-1}$ represents, in analogy to equation 32 ,

$$
\mathbf{J}^{-1}=\left[\begin{array}{ll}
\frac{\partial \xi}{\partial x} & \frac{\partial \xi}{\partial t} \\
\frac{\partial \eta}{\partial x} & \frac{\partial \eta}{\partial t}
\end{array}\right]
$$


The inverse Jacobian matrix is calculated by taking the matrix inverse of $\mathbf{J}$, which is particularly simple in two dimensions:

$$
\mathrm{J}^{-1}=\frac{1}{|\mathrm{~J}|}\left[\begin{array}{rr}
\frac{\partial t}{\partial \eta} & -\frac{\partial \mathrm{x}}{\partial \eta} \\
-\frac{\partial t}{\partial \xi} & \frac{\partial \mathrm{x}}{\partial \xi}
\end{array}\right] .
$$

$|J|$ is the determinant of the Jacobian matrix, which by equations 26 to 31 reduces here to

$$
|J|=\left(x_{2}-x_{1}\right)\left(t_{4}-t_{1}\right) / 4=\Delta x \Delta t / 4
$$

Global first derivatives of shape functions with respect to $x$ and $t$ are thus obtained from equation 34, which relates global derivatives to local derivatives with respect to $\xi$ and $\eta$ calculated from equations 12 to 21 , and given by

$$
\begin{aligned}
& \frac{\partial N_{1}^{h}}{\partial \xi}=(-1+2 \xi)(1-\eta) / 4 \\
& \frac{\partial N_{2}^{h}}{\partial \xi}=(1+2 \xi)(1-\eta) / 4
\end{aligned}
$$

$$
\frac{\partial N_{3}^{h}}{\partial \xi}=(1+2 \xi)(1+\eta) / 4
$$

$$
\frac{\partial N_{4}^{h}}{\partial \xi}=(-1+2 \xi)(1+\eta) / 4
$$

$$
\frac{\partial N_{5}^{h}}{\partial \xi}=-\xi(1-\eta)
$$

$\frac{\partial N_{6}^{h}}{\partial \xi}=-\xi(1+\eta)$

$\frac{\partial N_{1}^{h}}{\partial \eta}=\xi(1-\xi) / 4$

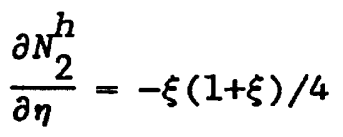

$$
\frac{\partial N_{3}^{h}}{\partial \eta}=\xi(1+\xi) / 4
$$

$\frac{\partial N_{4}^{h}}{\partial \eta}=-\xi(1-\xi) / 4$

$\frac{\partial N_{5}^{h}}{\partial \eta}=-\left(1-\xi^{2}\right) / 2$

$\frac{\partial N_{6}^{h}}{\partial \eta}=\left(1-\xi^{2}\right) / 2$

$\frac{\partial N_{1}^{Q}}{\partial \xi}=-(1-\eta) / 4$

$\frac{\partial N_{2}^{Q}}{\partial \xi}=(1-\eta) / 4$

$\frac{\partial N_{3}^{Q}}{\partial \xi}=(1+\eta) / 4$

$\frac{\partial N_{4}^{Q}}{\partial \xi}=-(1+\eta) / 4$

$\frac{\partial N_{1}^{Q}}{\partial \eta}=-(1-\xi) / 4$

$\frac{\partial N_{2}^{Q}}{\partial \eta}=-(1+\xi) / 4$

$\frac{\partial N_{3}^{Q}}{\partial \eta}=(1+\xi) / 4$

$\frac{\partial N_{4}^{Q}}{\partial \eta}=(1-\xi) / 4$.

Global second derivatives of the shape functions are required also. These are obtained by differentiating equation 34 , making use of the chain rule for differentiation. Second partial derivatives with respect to time $t$ are not actually used in the procedure, but are included for completeness; the second $t$-derivatives are all zero anyway, because of the linear time dependence. In the general case, we obtain 


$$
\begin{aligned}
& \frac{\partial}{\partial x}\left(\frac{\partial N}{\partial x}\right) \\
& =\frac{\partial^{2} N}{\partial \xi^{2}}\left[\frac{\partial \xi}{\partial x}\right]^{2}+2 \frac{\partial^{2} N}{\partial \xi} \frac{\partial \eta}{\partial \eta} \frac{\partial \xi}{\partial x} \\
& +\frac{\partial^{2} N}{\partial \eta^{2}}\left(\frac{\partial \eta}{\partial x}\right)^{2}+\frac{\partial N}{\partial \xi} \frac{\partial^{2} \xi}{\partial x^{2}}+\frac{\partial N}{\partial \eta} \frac{\partial^{2} \eta}{\partial x^{2}} \\
& \frac{\partial}{\partial t}\left(\frac{\partial N}{\partial t}\right) \\
& =\frac{\partial^{2} N}{\partial \xi^{2}}\left(\frac{\partial \xi}{\partial t}\right)^{2}+2 \frac{\partial^{2} N}{\partial \xi \partial \eta} \frac{\partial \eta}{\partial t} \frac{\partial \xi}{\partial t} \\
& +\frac{\partial^{2} N}{\partial \eta^{2}}\left(\frac{\partial \eta}{\partial t}\right)^{2}+\frac{\partial N}{\partial \xi} \frac{\partial^{2} \xi}{\partial t^{2}}+\frac{\partial N}{\partial \eta} \frac{\partial^{2} \eta}{\partial t^{2}} \\
& \frac{\partial}{\partial x}\left(\frac{\partial N}{\partial t}\right) \\
& =\frac{\partial^{2} N}{\partial \xi^{2}} \frac{\partial \xi}{\partial x} \frac{\partial \xi}{\partial t}+\frac{\partial^{2} N}{\partial \xi \partial \eta}\left(\frac{\partial \xi}{\partial x} \frac{\partial \eta}{\partial t}+\frac{\partial \xi}{\partial t} \frac{\partial \eta}{\partial x}\right) \\
& +\frac{\partial^{2} N}{\partial \eta^{2}} \frac{\partial \eta}{\partial x} \frac{\partial \eta}{\partial t}+\frac{\partial N}{\partial \xi} \frac{\partial^{2} \xi}{\partial x \partial t}+\frac{\partial N}{\partial \eta} \frac{\partial^{2} \eta}{\partial x \partial t},
\end{aligned}
$$

where once again $N_{j}$ represents either $N_{j}^{h}$ or $N_{j}^{Q}$. Because the transformation between global and local coordinates is linear, and because, for the special case being considered here, it is also separable ( $\xi$ is a function of $x$ alone, and $\eta$ is a function of $t$ alone, by equations 26 and 29), we have

$$
\begin{aligned}
& \frac{\partial^{2} \xi}{\partial x^{2}}=\frac{\partial^{2} \xi}{\partial t^{2}}=\frac{\partial^{2} \eta}{\partial x^{2}}=\frac{\partial^{2} \eta}{\partial t^{2}} \\
& =\frac{\partial^{2} \xi}{\partial x \partial t}=\frac{\partial^{2} \eta}{\partial x \partial t}=\frac{\partial \eta}{\partial x}=\frac{\partial \xi}{\partial t}=0 .
\end{aligned}
$$

Inserting equations 61,28 , and 31 into equations 58,59 , and 60 reduces the second partial derivatives for the special element geometry considered here to

$$
\begin{aligned}
& \frac{\partial^{2} N_{j}}{\partial x^{2}}=\frac{\partial^{2} N}{\partial \xi^{2}}\left(\frac{d \xi}{d x}\right)^{2} \\
& \frac{\partial^{2} N_{j}}{\partial t^{2}}=\frac{\partial^{2} N}{\partial \eta^{2}}\left(\frac{d \eta}{d t}\right)^{2} \\
& \frac{\partial^{2} N_{j}}{\partial t \partial x}=\frac{\partial^{2} N_{j}}{\partial \xi \partial \eta} \frac{d \xi}{d x} \frac{d \eta}{d t} .
\end{aligned}
$$

The second partial derivatives of $N_{j}$ with respect to local coordinates $\xi$ and $\eta$ are obtained by differentiating equations 38 to 57 to yield

$$
\begin{aligned}
& \frac{\partial^{2} N_{1}^{h}}{\partial \xi^{2}}=\frac{\partial^{2} N_{2}^{h}}{\partial \xi^{2}}=(1-\eta) / 2 \\
& \frac{\partial^{2} N_{3}^{h}}{\partial \xi^{2}}=\frac{\partial^{2} N_{4}^{h}}{\partial \xi^{2}}=(1+\eta) / 2 \\
& \frac{\partial^{2} N_{5}^{h}}{\partial \xi^{2}}=-(1-\eta) \\
& \frac{\partial^{2} N_{6}^{h}}{\partial \xi^{2}}=-(1+\eta)
\end{aligned}
$$

$$
\begin{aligned}
& \frac{\partial^{2} N_{1}^{h}}{\partial \eta^{2}}=\frac{\partial^{2} N_{2}^{h}}{\partial \eta^{2}}=\frac{\partial^{2} N_{3}^{h}}{\partial \eta^{2}} \\
& =\frac{\partial^{2} N_{4}^{h}}{\partial \eta^{2}}=\frac{\partial^{2} N_{5}^{h}}{\partial \eta^{2}}=\frac{\partial^{2} N_{6}^{h}}{\partial \eta^{2}}=0
\end{aligned}
$$

$$
\frac{\partial^{2} N_{1}^{h}}{\partial \xi \partial \eta}=-(-1+2 \xi) / 4
$$

$$
\frac{\partial^{2} N_{2}^{h}}{\partial \xi \partial \eta}=-(1+2 \xi) / 4
$$




$$
\begin{aligned}
& \frac{\partial^{2} N_{3}^{h}}{\partial \xi \partial \eta}=(1+2 \xi) / 4 \\
& \frac{\partial^{2} N_{4}^{h}}{\partial \xi \partial \eta}=(-1+2 \xi) / 4 \\
& \frac{\partial^{2} N_{5}^{h}}{\partial \xi \partial \eta}=\xi \\
& \frac{\partial^{2} N_{6}^{h}}{\partial \xi \partial \eta}=-\xi \\
& \frac{\partial^{2} N_{1}^{Q}}{\partial \xi^{2}}=\frac{\partial^{2} N_{2}^{Q}}{\partial \xi^{2}}=\frac{\partial^{2} N_{3}^{Q}}{\partial \xi^{2}}=\frac{\partial^{2} N_{4}^{Q}}{\partial \xi^{2}}=0 \\
& \frac{\partial^{2} N_{1}^{Q}}{\partial \eta^{2}}=\frac{\partial^{2} N_{2}^{Q}}{\partial \eta^{2}}=\frac{\partial^{2} N_{3}^{Q}}{\partial \eta^{2}}=\frac{\partial^{2} N_{4}^{Q}}{\partial \eta^{2}}=0 \\
& \frac{\partial^{2} N_{1}^{Q}}{\partial \xi \partial \eta}=\frac{\partial^{2} N_{3}^{Q}}{\partial \xi \partial \eta}=\frac{1}{4} \\
& \frac{\partial^{2} N_{2}^{Q}}{\partial \xi \partial \eta}=\frac{\partial^{2} N_{4}^{Q}}{\partial \xi \partial \eta}=-\frac{1}{4} .
\end{aligned}
$$

Approximations of channel parameters within the element are carried out by an independent procedure that will be described later in the development of the method. (See the section "Interpolation of Channel Parameters.") The approximation provides values within the elements, and variation across the elements, for channel parameters $A, B$, $P, n$, and $z_{b}$. These parameters are specified as input at cross-section locations that need not necessarily correspond to nodal locations such as $1,2,3,4,5$, and 6 of the computational elements.

\section{Characteristics}

The characteristics are approximated piecewise by straight-line segments $a b$ and $b c$, or $d e$ and $e f$ (fig. 3). The characteristics initiate at points a and $\mathrm{d}$, and are projected backwards according to equations 7 . The slope on each segment is averaged from the slopes given by the appropriate one of the equations 7 (upper or lower sign) at the end points of the segment; for example, at points $a$ and $b$ for segment $a b$, or $b$ and $c$ for segment $b c$. Figure 3 shows the situation for both subcritical and supercritical flow, as well as for transitions between two flow regimes. In figure 3 , the characteristics associated with the upper sign in equations 7 are denoted by + , and those associated with the lower sign by - .

The numerical process involves iteration, because although point $a$, for example, is at a fixed location, the location of point $b$ and the slope of the characteristic segment passing through it are both unknown at the outset. An initial approximation to the slope for the entire segment $a b$ is the slope from the appropriate one of equations 7 (upper sign, in this case) at point $a$. This locates an initial trial end point $b$ for the segment. Next, the slope of the characteristic at point $b$, from equations 7 , is averaged with the slope from point $a$ to obtain a new approximation for the slope of the segment. The process is repeated until convergence of point $b$ is obtained. Then the procedure used on segment $a b$ is repeated to track the characteristic through the next segment $b c$, with point $b$ taking the place of point $a$ and point $c$ replacing point $b$ in the algorithm.

In order to carry out the above "inner iteration" to determine the approximating characteristic path, the discharge $Q$ and depth $h$ must be known throughout the space-time element, and in particular at the nodes 3,4 , and 6 at the forward ${ }^{1}$ time (fig. 3 ). Because these nodal values are the quantities we seek to determine with the numerical algorithm, we must assume some initial values at the forward time to get the process started. These initial values for $Q$ and $h$ are taken to be identical to the values at the old time; that is, values at nodes 1,2 , and 5 are used as initial estimates at nodes 4,3 , and 6 , respectively. These estimates are improved by an "outer" Newton-Raphson iteration of the global system of equations to be described later in the section "System of Equations for the Finite Element Solution." (See equation 85.)

The number of segments $a b, b c$, and so on is adjustable, but two appear to be sufficient for the numerical procedure being described. The segmented approximating path is restricted to a topological "neighborhood of restriction" lying within the element. In a manner analogous to the forward time-weighting of the 4-point implicit numerical procedure (see, for example, Cunge and others, 1980 , p. 65), a parameter $\theta \in[0,1]$ is used to set the base of the neighborhood of restriction at local time coordinate $\eta=1-2 \theta$. By an additional parameter $\chi$, the characteristic starting at point $a$ (= element node 3 ) is restricted to the right of local coordinate $\xi=1-2 \chi$, and the characteristic starting at point $d$ (= element node 4 ) is restricted to the left of $\xi=-1+2 \chi$. Two neighborhoods of restriction are defined by this procedure, namely $3 A B C$ for characteristic $a b c$, and

${ }^{1}$ The terms "forward" and "new" are used to indicate the time level of nodes 3, 4, and 6; "old" denotes the time level of nodes 1,2 , and 5 . 


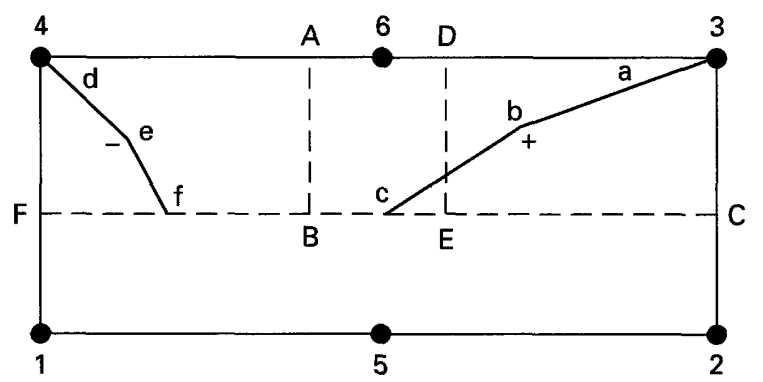

Local element - subcritical case

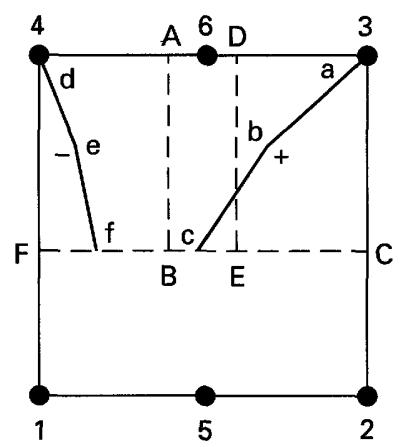

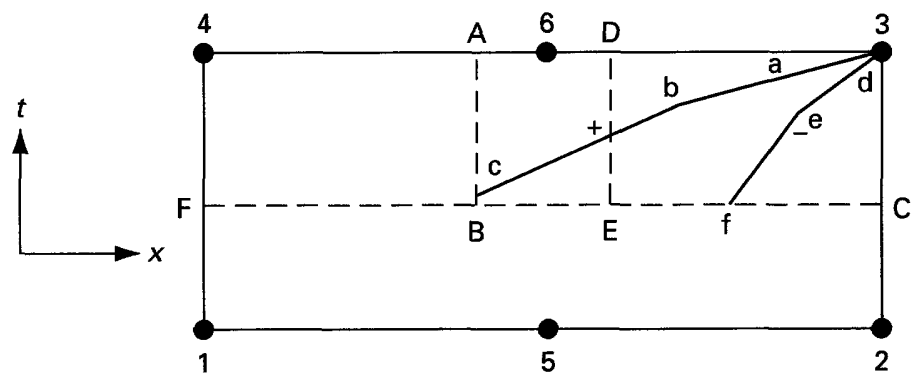

Local element - supercritical case

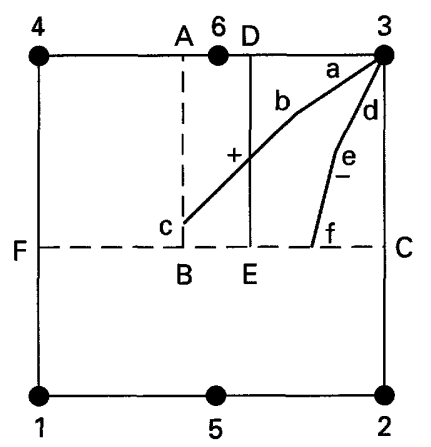

Transition - Subcritical to supercritical to subcritical flow across six global elements
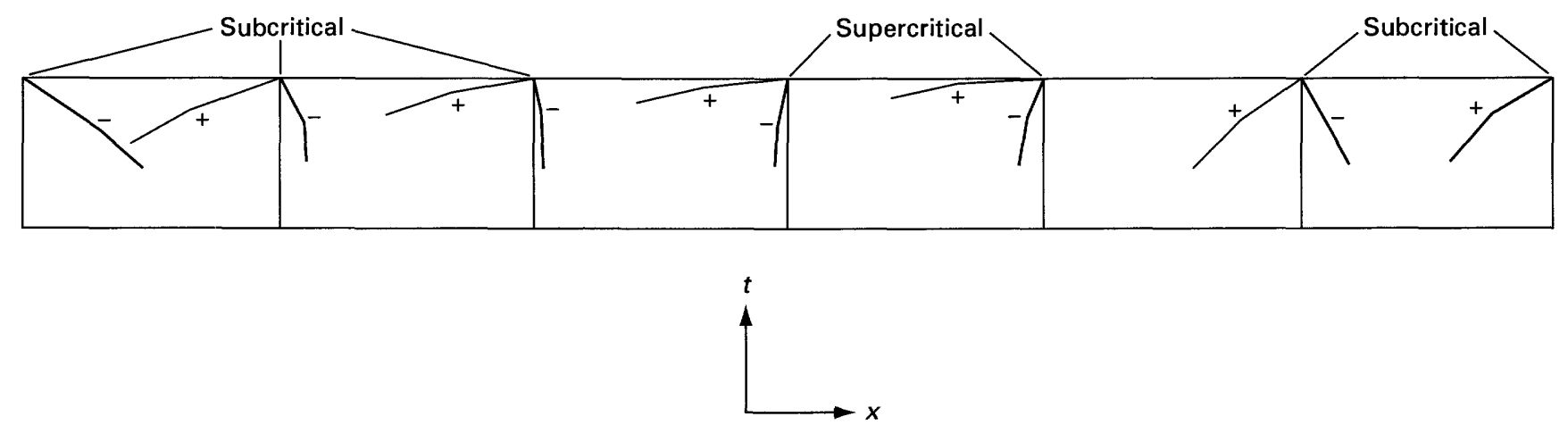

Figure 3. Approximation of characteristic paths. Dashed lines indicate the neighborhoods of restriction.

$4 D E F$ for characteristic $\operatorname{def}$ (fig. 3). In a manner somewhat similar to the choice of Galerkin weighting by shape functions that are unity at nodes 3 and 4 and that fall to zero at other nodes, these neighborhoods provide a weighting of the numerical scheme closer to the location of the unknown values of discharge $Q$ and water depth $h$ at nodes 3 and 4 . The neighborhoods of restriction thus specify a form of subdomain collocation. (See, for example, Zienkiewicz, 1977, p. 50.) The effect of the parameters $\chi$ and $\theta$ will be demonstrated after examples applying the model are introduced later in the report. (See the section "Example 2.")

The lengths of segments such as $a b$ and $b c$ are chosen by the following procedure, which prevents the segments from becoming too long either horizontally or vertically. Divide the interval $\eta_{C}$ to $\eta_{3}$, that is, from the base to top of the neighborhood $3 A B C$, into equal parts $\triangle \eta$ (two parts, for three characteristic nodal points shown in the figure). Also divide the interval $\xi_{A}$ to $\xi_{3}$ into equal parts $\Delta \xi$ (two parts, for the case in fig. 3). The local time interval $\eta_{a}-\eta_{b}$ along segment $a b$ is equal to $\Delta \eta$, unless this choice of time extent for $a b$ would make the corresponding space interval $\left|\xi_{a}-\xi_{b}\right|$ more than $\Delta \xi$. The latter situation occurs if the time steps are large enough that the slope of the characteristic in local coordinates is mild. If $\Delta \xi$ is exceeded using the full $\Delta \eta$ time interval, the segment $a b$ of the approximation to the characteristic is truncated so that $\left|\xi_{\mathrm{a}}-\xi_{\mathrm{b}}\right|$ equals $\Delta \xi$, in which case $\eta_{a}-\eta_{b}$ will be less than $\Delta \eta$. This procedure is then repeated for the continuing segment $b c$ (and possibly more segments) until the path intersects the neighborhood of restriction, where the path is 
truncated. The procedure occasionally can result in more than the minimum number of segments (two, in the case of fig. 3) being required to terminate on the boundary of the neighborhood of restriction. Such special cases occur rarely when the procedure just described switches between using $\Delta \eta$ and $\Delta \xi$ to determine the length of segments within the approximating path.

In order to approximate numerically that equations 8 are satisfied along the corresponding characteristics of equations 7 , we form the following weighted integral along the characteristics:

$$
\begin{gathered}
F_{j \pm}(Q, h)=\int_{\eta_{j \pm}}^{\eta_{j \pm}^{i}}\left[\dot{Q}_{ \pm}-\dot{x}_{\mp} B \dot{h}_{ \pm}-q\left(u_{q}-\dot{x}_{\mp}\right)\right. \\
\left.-u^{2} \frac{\partial A^{*}}{\partial x}+g A\left(s_{f}+\frac{\partial z_{b}}{\partial x}\right)\right] W_{j \pm} \frac{\partial t}{\partial \eta} d \eta
\end{gathered}
$$

The path of the characteristic is thus parameterized in terms of local time coordinate $\eta$, and the limits of integration range from $\eta_{j \pm}^{v}$ at the node $j$, backwards in time along the positive $(+)$ or negative $(-)$ characteristic to local coordinate $\eta_{j \pm}^{i}$. Node $j$ is restricted to one of the upper corners of the element, namely node 3 or 4 , and the superscript $v$ refers to the vertex formed at node $j$ by the space-time curves of dependence that follow the positive and negative characteristics backwards in time from the node. The characteristic can exit its neighborhood of restriction at the neighborhood's base $B C$ or $F E$; in this case $\eta_{j \pm}^{i}=1-2 \theta$. The exit can also be through the vertical sides $A B$ or $D E$, or (rarely) through vertical sides $3 C$ or $4 F$, and in this case $\eta_{j \pm}^{i}$ is between $1-2 \theta$ and +1 . In any case, the integral in equation 80 is restricted to that part of the path of the characteristic that lies totally within the neighborhood of restriction, which in turn lies within the element. The superscript $i$ refers to the intercept of the characteristic with the boundary of the neighborhood of restriction. The function $W_{j \pm}$ specifies the weighting; here, $W_{j \pm}$ is chosen to be unity along the entire positive or negative characteristic passing through node $j$. Note that when considered in the context of the classical two-dimensional weighted integral of the finite element method, use of a one-dimensional integration path following the characteristic corresponds to use of a restricted measure, which is further restricted by truncation of the path to lie within the neighborhood of restriction.

\section{Addition of Continuity Equation for Mass Conservation}

As indicated in the introduction, mass is not conserved in general with the standard numerical implementation of the method of characteristics that relies on equations 7 alone. The equations 8 are formally equivalent to the pair of equations 1 and 2 . This equivalence is true, however, only if the correspondence is enforced at every point of the space-time continuum. Because enforcement over the continuum is impossible in numerical approximation with a finite number of nodes, nonconservation can result, even with quadratic or cubic interpolation of all associated variables. To ensure the correspondence of equations 8 to equations 1 and 2, an additional equation that specifies mass conservation is enforced. The equation is provided by integrating continuity equation 1 over the local space-time element to obtain

$$
F_{c}(Q, h)=\int_{-1}^{1} \int_{-1}^{1}\left[B \frac{\partial h}{\partial t}+\frac{\partial Q}{\partial x}-q\right]|\mathrm{J}| d \xi \quad d \eta=0
$$

where $|\mathbf{J}|$ is the determinant of the Jacobian matrix $\mathbf{J}$ between the global and local space-time elements from equation 37 .

The addition of this equation in each element uses the degree of freedom that was provided by specifying quadratic instead of linear variation with distance for water depth $h$ in equation 11 .

\section{DESCRIPTION OF THE EQUATIONS FOR NUMERICAL SOLUTION}

\section{System of Equations for the Finite Element Solution}

A finite element solution is obtained by requiring, from equations 80 and 81 , that

$$
F_{j \pm}(Q, h)=0
$$

and

$$
F_{c}(Q, h)=0
$$

where $Q$ and $h$ are given by their finite element approximations (equations 10 and 11). Because the integrands in equations 80 and 81 are nonlinear functions of the nodal unknowns $a_{k}^{Q}$ and $a_{k}^{h}$, Newton-Raphson iteration on the approximating linear system is used. Let $\mathbf{a}^{Q}=$ column vector of nodal discharges, $\mathbf{a}^{h}=$ column vector of nodal water depths, and $\mathbf{a}^{T}=\left(\left(\mathbf{a}^{Q}\right)^{T},\left(\mathbf{a}^{h}\right)^{T}\right)=$ row vector of dependent nodal variables. (The superscript $T$ indicates transpose.) Let $\mathbf{F}(\mathbf{a})=$ column vector of integrals $\hat{F}_{j \pm}\left(\mathbf{a}^{Q}, \mathbf{a}^{h}\right) \equiv F_{j \pm}(Q, h)$ along characteristics from equation 80 , and integrals $\hat{F}_{c}\left(\mathbf{a}^{Q}, \mathbf{a}^{h}\right) \equiv$ 
$F_{c}(Q, h)$ over the space-time element from equation $81 .\left(F_{j \pm}\right.$ and $F_{c}$ are scalar-valued integrodifferential operators on functions $Q$ and $h$, whereas $\hat{F}_{j \pm}$ and $\hat{F}_{c}$ are regarded as functions of the nodal variables $\mathbf{a}^{Q}$ and $\mathbf{a}^{h}$.) In vector notation, the nonlinear system of equations to solve is

$$
F(a)=0 \text {, }
$$

and the approximating linear system is

$$
F(a)+F^{\prime}(a) \delta a \approx 0 \text {. }
$$

In equation $85, \mathbf{F}^{\prime}(\mathbf{a})$ is a matrix with elements of the form $\frac{\partial \hat{F}_{j \pm}}{\partial a_{k}^{Q}}, \frac{\partial \hat{F}_{j \pm}}{\partial a_{k}^{h}}, \frac{\partial \hat{F}_{c}}{\partial a_{k}^{Q}}$, and $\frac{\partial \hat{F}_{c}}{\partial a_{k}^{h}}$, and $\delta \mathbf{a}$ is a column vector of variations of the nodal variables from the trial values given by vector $\mathbf{a}$.

Equations 84 and 85 are treated as systems on individual finite elements during their formation, but can be assembled into global systems by collecting the equations from all elements in a river reach. For an $\mathrm{N}$-element reach, the global form of equation 84 or 85 includes $2 N+1$ unknowns $h$, and $N+1$ unknowns $Q$, for a total of $3 N+2$ unknowns. Calculations for this reach would include $2 N$ equations derived from equation $82, N$ equations derived from equation 83 , and 2 boundary conditions specified at the upper and (or) lower ends of the reach, for a total of $3 N+2$ equations, making the global system corresponding to equation 84 or 85 determinate.

\section{General Form of Partial Derivatives for Equations Along Characteristics}

Computation of the partial derivatives is carried out in the standard $2 \times 2$ finite element in independent variables $(\xi, \eta)$. Let the part of the integrand between the brackets in equation 80 be denoted by $\hat{F}_{g \pm}\left(\mathbf{a}^{Q}, \mathbf{a}^{h}, x, t\right) \equiv\left[F_{g \pm}(Q, h)\right](x, t)$, where $F_{g \pm}$ is a differential operator on functions $Q$ and $h$ in independent variables $x$ and $t$, and $\hat{F}_{g \pm}$ is regarded as a function of nodal variables $\mathbf{a}^{Q}, \mathbf{a}^{h}, x$, and $t$. Note that the integral of equation 80 is dependent on the path of the characteristic as given by equations 7 , and therefore, for any time $t$, the space coordinate $x$ at which integrand $\hat{F}_{g \pm}$ is evaluated also depends on nodal variables $\mathbf{a}^{Q}$ and $\mathbf{a}^{h}$. Then the partial derivatives $\frac{\partial \hat{F}_{j \pm}}{\partial a_{k}^{\varrho}}, \frac{\partial \hat{F}_{j \pm}}{\partial a_{k}^{h}}$ appearing in $\mathbf{F}^{\prime}(\mathbf{a})$ are given by

$$
\begin{aligned}
\frac{\partial \hat{F}_{j \pm}}{\partial a_{k}} & =\int_{\eta}^{\eta_{j \pm}}{ }^{i} \frac{\partial \hat{F}_{g \pm}}{\partial a_{k}} w_{j \pm} \frac{d t}{d \eta} d \eta \\
& +\int_{\eta_{j \pm}}^{\eta_{j \pm}^{i}} \frac{\partial \hat{F}_{g \pm}}{\partial x} w_{j \pm} \frac{d x}{d \xi} \frac{\partial \xi}{\partial a_{k}} \frac{d t}{d \eta} d \eta \\
& +\int_{j \pm}^{\eta_{j \pm}^{i}} \hat{F}_{g \pm} \frac{\partial W_{j \pm}}{\partial x} \frac{d x}{d \xi} \frac{\partial \xi}{\partial a_{k}} \frac{d t}{d \eta} d \eta \\
& +\left.\frac{\partial \eta_{j \pm}^{i}}{\partial a_{k}}\left[\hat{F}_{g \pm} w_{j \pm} \frac{d t}{d \eta}\right]\right|_{\eta} ^{i} .
\end{aligned}
$$

In equation $86, a_{k}$ represents either a nodal discharge $a_{k}^{Q}$ or a nodal water depth $a_{k}^{h}$. The first term in the equation is the main variation, obtained under the assumption of a fixed path for the characteristic. The second and third terms take into account the variation of the path of the characteristic, but under the assumption of a fixed intercept $\eta_{j \pm}^{i}$ with the element boundary. The third term is zero under the assumption of a constant weighting function that is taken here. The fourth term represents the contribution due to the variation of the integration limit $\eta_{j \pm}^{i}$. If the path of the characteristic is through the bottom of the neighborhood of restriction of figure 3 , where $\eta_{j \pm}^{i}=1-2 \theta$ and $\xi_{j \pm}^{i} \in(-1,1)$, this term is zero. In the linear iteration defined by equation 85 , its contribution is omitted even when the exit of the characteristic path is through the vertical sides $A B, D E, 3 C$, or $4 F$. Note that for the exact solution of the system of equations 7 and 8 , the integrand in equation 80 is identically zero along characteristics, regardless of the integration limits $\eta_{j \pm}^{v}$ and $\eta_{j \pm}^{i}$. The choice of limits and interpolations for the variables of the integrand only serve to define the numerical approximation of the solution. The variation of the characteristic path with $\delta \mathbf{a}$ is treated as confined between fixed $\eta$ coordinates during each iteration. (See fig. 4.) Then the improved approximation to the characteristic path determined at the beginning of the next iteration is again truncated to lie within the appropriate neighborhood of restriction. This procedure avoids computational difficulties, especially with near-vertical characteristic paths encountered close to critical flow, and still provides sufficient direction in nodal space a, through equation 85 , 


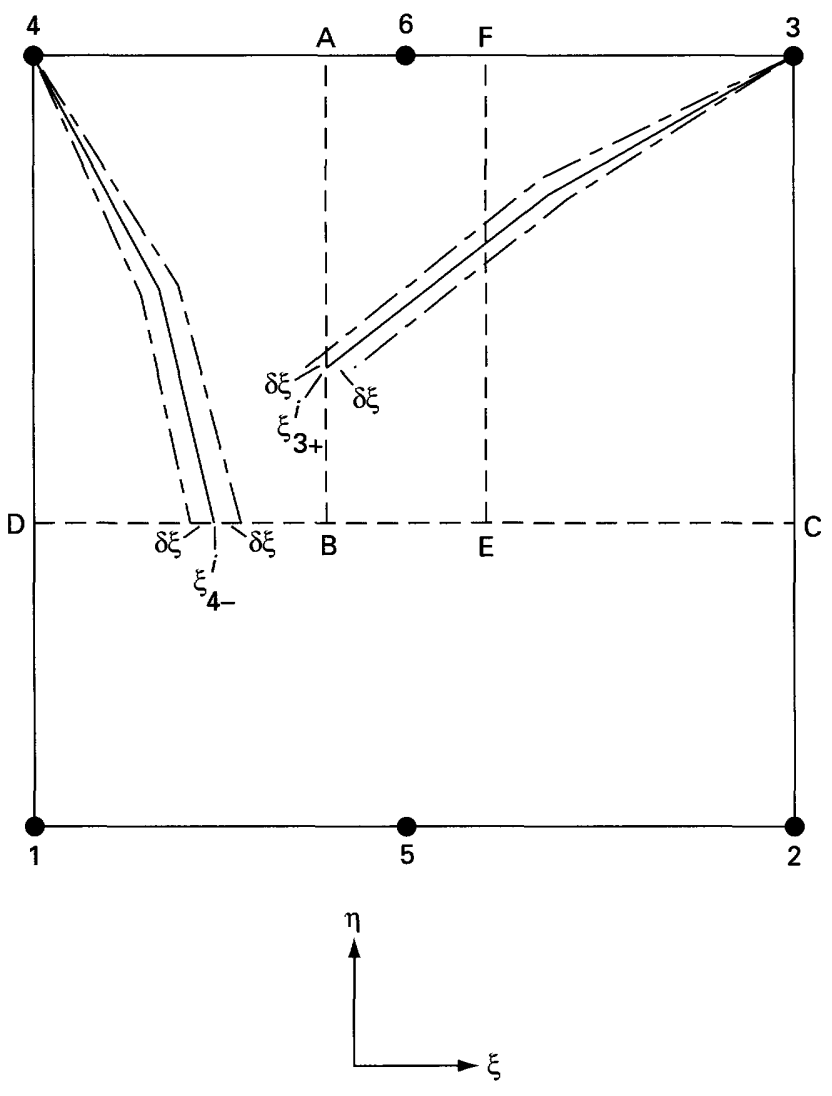

Figure 4. Variation of characteristic paths.

for convergence to the solution of the nonlinear system, equation 84 .

The integrals along characteristics in equations 80 and 86 are carried out numerically by trapezoidal rule integration along the piecewise linear approximation of the characteristic path in the local space-time element. If $I_{ \pm}(\xi, \eta)$ represents one of the integrands in equation 80 or 86 , the numerical quadrature is

$$
\begin{aligned}
& \int_{\eta_{j \pm}^{\eta}}^{\eta_{j \pm}^{i}} I_{ \pm}(\xi(\eta), \eta) d \eta \\
& \approx \sum_{\ell=2}^{\ell} \frac{1}{2}\left[I_{ \pm}\left(\xi_{\ell}, \eta_{\ell}\right)+I_{ \pm}\left(\xi_{\ell-1}, \eta_{\ell-1}\right)\right] \Delta \eta_{\ell}
\end{aligned}
$$

where $\left(\xi_{\ell}, \eta_{\ell}\right)$ is a node of the approximating characteristic path, $\Delta \eta_{\ell}=\eta_{\ell}-\eta_{\ell-1}$ is the local time increment for the segment, $\eta_{1}=\eta_{j \pm}^{v}$, and $\eta_{\ell_{\max }}=\eta_{j \pm}^{i}$.

\section{Partial Derivatives with Characteristic Path Fixed}

Let $\Gamma$ denote the path of the characteristic. The partial derivatives corresponding to the first term in equation 86 , under the assumption of a fixed characteristic path, are

$$
\begin{aligned}
& {\left[\frac{\partial F}{\partial a_{k}^{Q}}\right]_{\Gamma_{\text {fixed }}}} \\
& -\int_{\eta_{j \pm}}^{\eta_{j \pm}^{i}}\left\{\left[\frac{\partial Q}{\partial x}-\dot{x} \frac{\partial h}{\partial x}\right]_{\partial a_{k}^{Q}}^{\frac{\partial \dot{x}}{ \pm}}+\left[-B \dot{h}_{ \pm}+q\right] \frac{\partial \dot{x}}{\partial a_{k}^{Q}}\right. \\
& +\left[-2 u \frac{\partial A^{*}}{\partial x}\right] \frac{\partial u}{\partial a_{k}^{Q}}+\left[g \frac{\partial}{\partial Q}\left(A S_{f}\right)\right] \frac{\partial Q}{\partial a_{k}^{Q}} \\
& \left.+\left[\dot{\mathrm{x}}_{ \pm}\right] \frac{\partial}{\partial a_{k}^{Q}}\left(\frac{\partial Q}{\partial x}\right)+[1] \frac{\partial}{\partial a_{k}^{Q}}\left(\frac{\partial Q}{\partial t}\right)\right\}_{j \pm} \frac{d t}{d \eta} d \eta,
\end{aligned}
$$

and

$$
\left[\frac{\partial F i \pm}{\partial a_{k}^{h}}\right]_{\Gamma_{f i x e d}}
$$$$
=\int_{\eta_{j \pm}}^{\eta_{j \pm}^{j}}\left\{\left[\frac{\partial Q}{\partial x}-\dot{x}_{\mp} B \frac{\partial h}{\partial x}\right] \frac{\partial \dot{x}_{ \pm}}{\partial a_{k}^{h}}+\left[-B \dot{h}_{ \pm}+q\right] \frac{\partial \dot{x}_{\mp}}{\partial a_{k}^{h}}\right.
$$$$
+\left[-2 u \frac{\partial A^{*}}{\partial x}\right] \frac{\partial u}{\partial a_{k}^{h}}+\left[-\dot{x}_{F} \dot{h}_{ \pm}\right] \frac{\partial B}{\partial a_{k}^{h}}
$$$$
+\left[g \frac{\partial z_{b}}{\partial x}+g \frac{\partial\left(A S_{f}\right)}{\partial A}\right] \frac{\partial A}{\partial a_{k}^{h}}+\left[g \frac{\partial\left(A S_{f}\right)}{\partial P}\right] \frac{\partial P}{\partial a_{k}^{h}}
$$

$+\left[g \frac{\partial\left(A S_{f}\right)}{\partial n}\right] \frac{\partial n}{\partial a_{k}}+\left[-\dot{x}_{\mp} B \dot{x}_{ \pm}\right] \frac{\partial}{\partial a_{k}^{h}}\left(\frac{\partial h}{\partial \mathrm{x}}\right)$ 


$$
\begin{aligned}
& +\left[-\dot{x}_{F} B\right] \frac{\partial}{\partial a_{k}^{h}}\left(\frac{\partial h}{\partial t}\right)+\left[-u^{2}\right]_{\partial a_{k}^{h}}\left(\frac{\partial A^{*}}{\partial x}\right) \\
& \left.+\left[g \frac{\partial\left(A S_{f}\right)}{\partial S_{w}}\right]_{\partial a_{k}^{h}}^{\partial S_{w}}\right\}_{j \pm} \frac{d t}{d \eta} d \eta .
\end{aligned}
$$

In deriving equations 88 and 89 , the following expressions for total time derivatives along the characteristics were used:

$$
\begin{aligned}
& \dot{Q}_{ \pm}=\dot{x}_{ \pm} \frac{\partial Q}{\partial x}+\frac{\partial Q}{\partial t} \\
& \dot{h}_{ \pm}=\dot{x}_{ \pm} \frac{\partial h}{\partial x}+\frac{\partial h}{\partial t}
\end{aligned}
$$

The friction slope in equations 88 and 89 is specified by Manning's relation $S_{f_{0}}$, modified by a steep-slope factor $\Phi$ (Jarrett, 1984; Glass and others, 1987):

$$
s_{f}=S_{f_{0}} \Phi,
$$

where

Factor $\Phi$ is a function of the water surface slope $S_{w}$.

$$
S_{f_{0}}=\frac{n^{2}|Q| Q P^{4 / 3}}{2.208 A^{10 / 3}}
$$

The friction slope reduces to $S_{f_{0}}$ for mild slopes, because $\Phi=1$ if $S_{w} \leq 0.002$. More detail about the factor $\Phi$ for high-gradient streams is given below, in the section "SteepSlope Factor." In equation 93, $n$ is Manning's coefficient, and $P$ is the wetted perimeter. The variables in equations 88 and 89 are expressed in finite element formulation as follows. From equation 10, giving the linear combination of shape functions for discharge $Q$, we obtain

$$
\begin{aligned}
& \frac{\partial Q}{\partial a_{k}^{Q}}=N_{k}^{Q} \\
& \frac{\partial Q}{\partial x}=\sum_{j=1}^{4} \frac{\partial N_{j}^{Q}}{\partial x} a_{j}^{Q} \\
& \frac{\partial}{\partial a_{k}^{Q}}\left(\frac{\partial Q}{\partial x}\right)=\frac{\partial N_{k}^{Q}}{\partial x}
\end{aligned}
$$

$$
\frac{\partial Q}{\partial t}=\sum_{j=1}^{4} \frac{\partial N_{j}^{Q}}{\partial t} a_{j}^{Q}
$$

$$
\frac{\partial}{\partial a_{k}^{Q}}\left(\frac{\partial Q}{\partial t}\right)=\frac{\partial N_{k}^{Q}}{\partial t} .
$$

Because they will be needed in the next section, which details the second term in equation 86 , we also have the following partial derivatives with respect to river coordinate $x$ :

$$
\begin{aligned}
& \frac{\partial}{\partial x}\left(\frac{\partial Q}{\partial x}\right)=\sum_{j=1}^{4} \frac{\partial^{2} N_{j}^{Q}}{\partial x^{2}} a_{j}^{Q=0} \\
& \frac{\partial}{\partial x}\left(\frac{\partial Q}{\partial t}\right)=\sum_{j=1}^{4} \frac{\partial^{2} N_{j}^{Q}}{\partial x \partial t} a_{j}^{Q} .
\end{aligned}
$$

The second partial derivative in equation 99 is zero, by equations 62 and 76 , because the shape functions $N_{j}^{Q}$ are bilinear.

From equation 11, giving the linear combination of shape functions for water depth $h$, we obtain

$$
\begin{aligned}
& \frac{\partial h}{\partial a_{k}^{h}}=N_{k}^{h} \\
& \frac{\partial h}{\partial x}-\sum_{j=1}^{6} \frac{\partial N_{j}^{h}}{\partial x^{h}} a_{j}^{h}
\end{aligned}
$$

$$
\frac{\partial}{\partial a_{k}^{h}}\left(\frac{\partial h}{\partial x}\right)=\frac{\partial N_{k}^{h}}{\partial x}
$$

$$
\frac{\partial h}{\partial t}=\sum_{j=1}^{6} \frac{\partial N_{j}^{h}}{\partial t} a_{j}^{h}
$$

$$
\frac{\partial}{\partial a_{k}^{h}}\left(\frac{\partial h}{\partial t}\right)=\frac{\partial N_{k}^{h}}{\partial t}
$$


Again, in anticipation of derivatives needed in the second term of equation 86 , we have

$$
\begin{aligned}
& \frac{\partial}{\partial x}\left(\frac{\partial h}{\partial x}\right)=\sum_{j=1}^{6} \frac{\partial^{2} N_{j}^{h}}{\partial x^{2}} a_{j}^{h} \\
& \frac{\partial}{\partial x}\left(\frac{\partial h}{\partial t}\right)=\sum_{j=1}^{6} \frac{\partial^{2} N_{j}^{h}}{\partial x \partial t} a_{j}^{h}
\end{aligned}
$$

We obtain partial derivatives of channel parameters $A, B, P$, and $n$ with respect to the nodal variables as follows. In a manner similar to equation 3 for cross-sectional area, we express the top width, wetted perimeter, and Manning's coefficient in the functional forms

$$
\begin{aligned}
& B(x, t)-B^{*}(x, h(x, t)) \\
& P(x, t)-P^{*}(x, h(x, t)) \\
& n(x, t)-n^{*}(x, h(x, t)) .
\end{aligned}
$$

Note that although Manning's $n$ can be related to other quantities such as discharge, the most typical implementation in practice is to have it vary along the stream. Practiced observers thus make estimates of channel roughness at various stream locations. At such data cross sections, they might also specify the coefficient directly as a function of water depth, or estimate its values across the width of the stream. The latter case can be converted approximately to a depth-dependent specification by one of the "composite roughness" formulas. (See Chow, 1959, p. 136-140, for example.) We restrict the dependence of Manning's coefficient to that given by equation 110 .

Then the partial derivatives are

$$
\begin{gathered}
\frac{\partial A}{\partial a_{k}^{Q}}=\frac{\partial B}{\partial a_{k}^{Q}}=\frac{\partial P}{\partial a_{k}^{Q}}=\frac{\partial n}{\partial a_{k}^{Q}}=0 \\
\frac{\partial A}{\partial a_{k}^{h}}=\frac{\partial A^{*}}{\partial h} \frac{\partial h}{\partial a_{k}^{h}}=B \frac{\partial h}{\partial a_{k}^{h}} \\
\frac{\partial B}{\partial a_{k}^{h}}=\frac{\partial B}{\partial h} \frac{\partial h}{\partial a_{k}^{h}}
\end{gathered}
$$

$$
\begin{gathered}
\frac{\partial P}{\partial a_{k}^{h}}=\frac{\partial P^{*}}{\partial h} \frac{\partial h}{\partial a_{k}^{h}} \\
\frac{\partial n}{\partial a_{k}^{h}}=\frac{\partial n^{*}}{\partial h} \frac{\partial h}{\partial a_{k}^{h}} \\
\frac{\partial a_{k}^{h}\left(\frac{\partial A^{*}}{\partial x}\right)=}{\frac{\partial}{\partial h}\left(\frac{\partial A^{*}}{\partial x}\right) \frac{\partial h}{\partial a_{k}^{h}}} \\
=\frac{\partial}{\partial x}\left(\frac{\partial A^{*}}{\partial h}\right) \frac{\partial h}{\partial a_{k}^{h}}=\frac{\partial B^{*}}{\partial x} \frac{\partial h}{\partial a_{k}^{h}} \\
\frac{\partial}{\partial a_{k}^{Q}}\left(\frac{\partial A^{*}}{\partial x}\right)-0 .
\end{gathered}
$$

For use in the second term of equation 86 , partial derivatives of channel parameters $B, P$, and $n$ with respect to $x$ are similar to equation 4 for the $x$-derivative of area $A$ :

$$
\begin{aligned}
& \frac{\partial B}{\partial x}=\frac{\partial B^{*}}{\partial x}+\frac{\partial B^{*}}{\partial h} \frac{\partial h}{\partial x} \\
& \frac{\partial P}{\partial x}=\frac{\partial P^{*}}{\partial x}+\frac{\partial P^{*}}{\partial h} \frac{\partial h}{\partial x} \\
& \frac{\partial n}{\partial x}=\frac{\partial n^{*}}{\partial x}+\frac{\partial n^{*}}{\partial h} \frac{\partial h}{\partial x}
\end{aligned}
$$

The partial derivatives of $A^{*}, B^{*}, P^{*}$, and $n^{*}$ with respect to $h$ and $x$ will be described later in the section "Interpolation of Channel Parameters."

Partial derivatives of the average velocity

$$
\mathbf{u}=\mathrm{Q} / \mathrm{A}
$$

with respect to nodal variables are given by

$$
\begin{aligned}
& \frac{\partial u}{\partial a_{k}^{Q}}=\frac{\partial u}{\partial Q} \frac{\partial Q}{\partial a_{k}^{Q}}=\frac{1}{A} \frac{\partial Q}{\partial a_{k}^{Q}} \\
& \frac{\partial u}{\partial a_{k}^{h}}=\frac{\partial u}{\partial A} \frac{\partial A}{\partial a_{k}^{h}}=-\frac{Q}{A^{2}} \frac{\partial A}{\partial a_{k}^{h}} .
\end{aligned}
$$


Again in anticipation of describing the second term in equation 86 , the partial derivative of the average velocity with respect to river distance is

$$
\begin{aligned}
\frac{\partial u}{\partial x} & =\frac{\partial u}{\partial Q} \frac{\partial Q}{\partial x}+\frac{\partial u}{\partial A} \frac{\partial A}{\partial x} \\
& =\frac{1}{A} \frac{\partial Q}{\partial x}-\frac{Q}{A} \frac{\partial A}{\partial x} .
\end{aligned}
$$

Partial derivatives for the critical speed

$$
C=\sqrt{g A / B}
$$

with respect to nodal variables, and with respect to $x$, are given by

$$
\begin{gathered}
\frac{\partial c}{\partial a_{k}^{Q}}=0 \\
\frac{\partial c}{\partial a_{k}^{h}}=\frac{\partial c}{\partial A} \frac{\partial A}{\partial a_{k}^{h}}+\frac{\partial c}{\partial B} \frac{\partial B}{\partial a_{k}^{h}} \\
=\frac{c}{2 A} \frac{\partial A}{\partial a_{k}^{h}}-\frac{c}{2 B} \frac{\partial B}{\partial a_{k}^{h}} \\
\frac{\partial c}{\partial x}=\frac{\partial c}{\partial A} \frac{\partial A}{\partial x}+\frac{\partial c}{\partial B} \frac{\partial B}{\partial x} \\
=\frac{c}{2 A} \frac{\partial A}{\partial x}-\frac{c}{2 B} \frac{\partial B}{\partial x} .
\end{gathered}
$$

Partial derivatives for the characteristic slope $\dot{x}_{ \pm}=u \pm c$ are

$$
\begin{aligned}
& \frac{\partial \dot{x}_{ \pm}}{\partial a_{k}^{Q}}=\frac{\partial u}{\partial a_{k}^{Q}} \\
& \frac{\partial \dot{x}_{ \pm}}{\partial a_{k}^{h}}=\frac{\partial u}{\partial a_{k}^{h}} \pm \frac{\partial c}{\partial a_{k}^{h}} \\
& \frac{\partial \dot{x}_{ \pm}}{\partial x}=\frac{\partial u}{\partial x} \pm \frac{\partial c}{\partial x} .
\end{aligned}
$$

Partial derivatives of the opposite characteristic slope $x_{\mp}=u \mp c$ are given by expressions similar to equations 129 , 130 , and 131, but with the plus and minus signs interchanged.
The partial derivatives of area $A$ times the friction slope $S_{f}=S_{f_{o}} \Phi$ are given by

$$
\begin{aligned}
& \frac{\partial\left(A S_{f}\right)}{\partial Q}=\Phi \frac{\partial\left(A S_{f_{0}}\right)}{\partial Q}=\Phi \frac{n^{2}|Q| P^{4 / 3}}{2.208 A^{7 / 3}} \\
& \frac{\partial\left(A S_{f}\right)}{\partial A}=\Phi \frac{\partial\left(A S_{f_{0}}\right)}{\partial A}=-\left(A S_{f}\right) \frac{7 / 3}{A}
\end{aligned}
$$
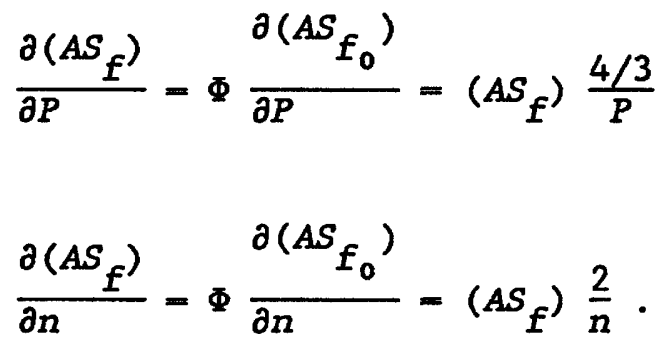

The dependence of $A S_{f}$ on water surface slope $S_{w}$ through the steep-slope factor $\Phi$ will be discussed in the section "Steep-Slope Factor."

\section{Partial Derivatives Due to Translation of the Characteristic Path}

The second term in equation 86 that arises because of variation in the path of the characteristic depends on partial derivatives of $F_{g \pm}$ with respect to river coordinate $x$ :

$$
\begin{aligned}
\frac{\partial F_{g \pm}}{\partial \mathrm{x}} & =\left[\frac{\partial Q}{\partial \mathrm{x}}-\dot{\mathrm{x}}_{\mp} B \frac{\partial h}{\partial \mathrm{x}}\right] \frac{\partial \dot{x}_{ \pm}}{\partial \mathrm{x}}+\left[-B \dot{h}_{ \pm}+q\right] \frac{\partial \dot{\mathrm{x}}_{\mp}}{\partial \mathrm{x}} \\
& +\left[-2 u \frac{\partial A^{*}}{\partial \mathrm{x}}\right] \frac{\partial u}{\partial \mathrm{x}}+\left[g \frac{\partial}{\partial Q}\left(A S_{f}\right)\right] \frac{\partial Q}{\partial \mathrm{x}} \\
& +\left[\dot{\mathrm{x}}_{ \pm}\right] \frac{\partial}{\partial \mathrm{x}}\left[\frac{\partial Q}{\partial \mathrm{x}}\right)+[1] \frac{\partial}{\partial \mathrm{x}}\left(\frac{\partial Q}{\partial t}\right) \\
& +\left[-\dot{\mathrm{x}}_{\mp} \dot{h}_{ \pm}\right] \frac{\partial B}{\partial \mathrm{x}}+\left[g \frac{\partial z_{b}}{\partial \mathrm{x}}+g \frac{\partial\left(A S_{f}\right)}{\partial A}\right] \frac{\partial A}{\partial \mathrm{x}} \\
& +\left[g \frac{\partial\left(A S_{f}\right)}{\partial P}\right] \frac{\partial P}{\partial \mathrm{x}}+\left[g \frac{\partial\left(A S_{f}\right)}{\partial n}\right] \frac{\partial n}{\partial \mathrm{x}} \\
& +\left[-\dot{x}_{\mp} B \dot{x}_{ \pm}\right] \frac{\partial}{\partial \mathrm{x}}\left(\frac{\partial h}{\partial \mathrm{x}}\right)+\left[-\dot{x}_{F} B\right] \frac{\partial}{\partial \mathrm{x}}\left(\frac{\partial h}{\partial t}\right)
\end{aligned}
$$

Description of the Equations for Numerical Solution $\quad 15$ 


$$
\begin{aligned}
& +\left[-u^{2}\right] \frac{\partial}{\partial x}\left(\frac{\partial A^{*}}{\partial x}\right)+\left[g \frac{\partial\left(A S_{f}\right)}{\partial S_{w}}\right] \frac{\partial S_{w}}{\partial x} \\
& +[g A] \frac{\partial}{\partial x}\left(\frac{\partial z_{b}}{\partial x}\right)
\end{aligned}
$$

In deriving equation 136, we have assumed that lateral inflow $q$ and its velocity component $u_{q}$ in the direction $x$ are both specified as piecewise constant functions, so that the derivatives of these quantities with respect to $x$ are zero except at a finite number of points. Equality to zero almost everywhere is sufficient because the derivatives in equation 136 appear under the integral sign in equation 86.

The second term in equation 86 also contains the factor $\partial \xi / \partial a_{k}$, which describes the variation of the path of the characteristic with changes in the nodal variables. To arrive at an expression for this factor, let $\dot{\xi}_{ \pm}$be the total $\eta$ derivative along the characteristic in local coordinates, corresponding to $\dot{x}_{ \pm}$in global coordinates. Note that $\xi_{ \pm}$is a field of characteristic directions in local coordinates $\xi$ and $\eta$, which is dependent on $a_{k}^{Q}$ and $a_{k}^{h}$ as well. Integrating along a characteristic yields $\xi$ as a function of $\eta$, where $\xi$ is also dependent on the nodal variables $a_{k}^{Q}$ and $a_{k}^{h}$ :

$$
\xi=\xi^{v}+\int_{\eta}^{\eta} \dot{\xi}_{ \pm} d \hat{\eta}
$$

The partial derivative with respect to nodal variable $a_{k}$, representing either $a_{k}^{Q}$ or $a_{k}^{h}$, is

$$
\frac{\partial \xi}{\partial a_{k}}=\frac{\partial \xi^{v}}{\partial a_{k}}+\int_{\eta}^{\eta}\left\{\frac{\partial \dot{\xi}_{ \pm}}{\partial a_{k}}+\frac{\partial \dot{\xi}_{ \pm}}{\partial \xi} \frac{\partial \xi}{\partial a_{k}}\right\} \hat{d \eta} .
$$

The first term on the right side of equation 138 is zero because the location of the vertex is fixed at one of the upper corners of the element. The partial derivatives appearing in the integrals are calculated as follows: The relation between the local and global field of characteristic directions is given by

$$
\dot{\xi}_{ \pm}=\dot{x}_{ \pm} \frac{d t}{d \eta} / \frac{d x}{d \xi}
$$

and from this we obtain

$$
\frac{\partial \dot{\xi}_{ \pm}}{\partial a_{k}}=\frac{\partial \dot{x}}{\partial a_{k}} \frac{d t}{d \eta} / \frac{d x}{d \xi}
$$

and

$$
\begin{aligned}
& \frac{\partial \dot{0}_{ \pm}}{\partial \xi}=\frac{\partial \dot{\xi}_{ \pm}}{\partial \mathrm{x}} \frac{d \mathrm{x}}{d \xi} \\
& =\frac{\partial \dot{x}_{ \pm}}{\partial \mathrm{x}}\left(\frac{d t}{d \eta} / \frac{d x}{d \xi}\right) \frac{d x}{d \xi}+\dot{x}_{ \pm} \frac{\partial}{\partial x}\left(\frac{d t}{d \eta} / \frac{d x}{d \xi}\right) \frac{d x}{d \xi}
\end{aligned}
$$

The partial derivative with respect to $x$ in the last term of equation 141 is zero because the mapping between global and local elements is linear. Equation 141 thus reduces to

$$
\frac{\partial \dot{\xi}_{ \pm}}{\partial \xi}=\frac{\partial \dot{x}_{ \pm}}{\partial \mathrm{x}} \frac{d t}{d \eta} .
$$

The calculation of equation 138 is carried out by numerical quadrature over each segment of the piecewiselinear approximation, starting at the vertex $\left(\xi^{v}, \eta^{v}\right)$. On each segment $\xi_{\in}\left[\xi_{\ell-1}, \xi_{l}\right], \eta \in\left[\eta_{\ell-1}, \eta_{\ell}\right]$, the appropriate integral to approximate numerically is similar to equation 138 :

$$
\begin{aligned}
\left.\frac{\partial \xi}{\partial a_{k}}\right|_{\ell} & =\left.\frac{\partial \xi}{\partial a_{k}}\right|_{\ell-1} \\
& +\int_{\eta_{\ell-1}}^{\eta_{\ell}}\left\{\frac{\partial^{\circ} \pm}{\partial a_{k}}+\frac{\partial \dot{\xi}_{ \pm}}{\partial \xi} \frac{\partial \xi}{\partial a_{k}}\right\} d \eta .
\end{aligned}
$$

The notation $\left.\right|_{\ell}$ is used to indicate evaluation at the point on the characteristic with local coordinates $\left(\xi_{\ell}, \eta_{\ell}\right)$, and $l_{\ell-1}$ indicates evaluation at point $\left(\xi_{\ell-1}, \eta_{\ell-1}\right)$. Using a trapezoidal rule approximation to the integral on the right side yields

$$
\begin{aligned}
& \left.\left.\frac{\partial \xi}{\partial a_{k}}\right|_{\ell} \approx \frac{\partial \xi}{\partial a_{k}}\right|_{\ell-1} \\
& +\left.\frac{\Delta \eta}{2} \frac{\partial \dot{\xi}_{ \pm}}{\partial a_{k}}\right|_{\ell-1}+\left.\left.\frac{\Delta \eta}{2} \frac{\partial \dot{\xi}_{ \pm}}{\partial \xi}\right|_{\ell-1} \frac{\partial \xi}{\partial a_{k}}\right|_{\ell-1} \\
& +\left.\frac{\Delta \eta}{2} \frac{\partial \dot{\circ}_{ \pm}}{\partial a_{k}}\right|_{\ell}+\left.\left.\frac{\Delta \eta}{2} \frac{\partial \dot{\leftrightarrow}_{ \pm}}{\partial \xi}\right|_{\ell} \frac{\partial \xi}{\partial a_{k}}\right|_{\ell},
\end{aligned}
$$


where $\Delta \eta=\eta-\eta{ }_{-1}$. Note that $\partial \xi /\left.\partial a_{k}\right|_{l}$ appears on both sides of equation 144. Collecting these terms gives

$\left.\frac{\partial \xi}{\partial a_{k}}\right|_{\ell} \approx\left(1-\left.\frac{\Delta \eta}{2} \frac{\partial \dot{o}_{ \pm}}{\partial \xi}\right|_{\ell}\right)^{-1}\left\{\left.\frac{\partial \xi}{\partial a_{k}}\right|_{\ell-1}\right.$

$+\left.\frac{\Delta \eta}{2} \frac{\partial \dot{\dot{\xi}}_{ \pm}}{\partial a_{k}}\right|_{\ell-1}+\left.\left.\frac{\Delta \eta}{2} \frac{\partial \dot{0}_{ \pm}}{\partial \xi}\right|_{\ell-1} \frac{\partial \xi}{\partial a_{k}}\right|_{\ell-1}$

$\left.+\left.\frac{\Delta \eta}{2} \frac{\partial \dot{\partial}_{ \pm}}{\partial a_{k}}\right|_{\ell}\right\}$

To start the process, note that the first node is a vertex at an upper corner of the element, so that

$$
\left.\frac{\partial \xi}{\partial a_{k}}\right|_{1}=0 \text {. }
$$

Equation 145 then iteratively gives $\partial \xi /\left.\partial a_{k}\right|_{\ell}$ for $\ell=2, \ldots$, $\ell_{\text {max }}$.

Equation 145 is not a good approximation if

$$
1-\left.\frac{\Delta \eta}{2} \frac{\partial \dot{0}_{ \pm}}{\partial \xi}\right|_{\ell} \leq 0
$$

A real application that would satisfy equation 147 appears unlikely, but an alternate numerical quadrature for equation 143 is nevertheless included to treat this case. For numerical reasons, bound the expression on the left side of equation 147 away from zero by a small amount. Use equation 145 if

$$
1-\left.\frac{\Delta \eta}{2} \frac{\partial \dot{\jmath}_{ \pm}}{\partial \xi}\right|_{\ell} \geq 0.001
$$

otherwise use the following numerical quadrature. First, define

$$
\begin{aligned}
& \overline{\frac{\partial \dot{\xi}_{ \pm}}{\partial a_{k}}}=\frac{1}{2}\left\{\left.\frac{\partial \dot{\circ}_{ \pm}}{\partial a_{k}}\right|_{\ell-1}+\left.\frac{\partial \dot{\sigma}_{ \pm}}{\partial a_{k}}\right|_{\ell}\right\} \\
& \overline{\frac{\partial \xi_{ \pm}}{\partial \xi}}=\frac{1}{2}\left\{\left.\frac{\partial \dot{\circ}_{ \pm}}{\partial \xi}\right|_{\ell-1}+\left.\frac{\partial \dot{\xi}_{ \pm}}{\partial \xi}\right|_{\ell}\right\} .
\end{aligned}
$$

Then, approximate integral 143 by

$$
\begin{aligned}
& \left.\left.\frac{\partial \xi}{\partial a_{k}}\right|_{\ell} \approx \frac{\partial \xi}{\partial a_{k}}\right|_{\ell-1} \\
& \quad+\int_{\eta_{\ell-1}}^{\eta_{\ell}}\left\{\overline{\frac{\partial \dot{\xi}_{ \pm}}{\partial a_{k}}}+\frac{\overline{\partial \dot{\xi}_{ \pm}}}{\partial \xi} \frac{\partial \xi}{\partial a_{k}}\right\} d \eta .
\end{aligned}
$$

Divide the interval $\left[\eta_{\ell-1}, \eta_{\ell}\right]$ into $m$ subintervals, where $m$ is chosen to satisfy

$$
\frac{\Delta \eta}{m} \frac{\overline{\partial \xi_{ \pm}}}{\partial \xi} \leq 1
$$

Require also that $m \geq 2$. Further, define

$$
\begin{aligned}
& \eta_{\ell, i}=\eta_{\ell-1}+\frac{i \Delta \eta}{m} \\
& \quad \text { for } \quad i=1,2, \ldots, m .
\end{aligned}
$$

Using the same approximations, equations 149 and 150 , which apply to the overall segment $\left[\eta_{\ell-1}, \eta_{\ell}\right]$, the restriction of equation 151 to the $i$ th subinterval is

$$
\begin{aligned}
& \left.\left.\frac{\partial \xi}{\partial a_{k}}\right|_{\ell, i} \approx \frac{\partial \xi}{\partial a_{k}}\right|_{\ell, i-1} \\
& +\int_{\eta_{\ell, i-1}}^{\eta_{\ell, i}}\left\{\begin{array}{l}
\overline{\frac{\partial \xi_{ \pm}}{\partial a_{k}}}+\frac{\partial \dot{\circ}_{ \pm}}{\partial \xi} \frac{\partial \xi}{\partial a_{k}}
\end{array}\right\} d \eta .
\end{aligned}
$$

By the trapezoidal rule,

$$
\begin{aligned}
& \left.\left.\frac{\partial \xi}{\partial a_{k}}\right|_{\ell, i} \approx \frac{\partial \xi}{\partial a_{k}}\right|_{\ell, i-1}+\frac{\Delta \eta}{m} \frac{\overline{\frac{\partial \xi_{ \pm}}{m}}}{\partial a_{k}} \\
& +\frac{\overline{2 \eta}}{\frac{\partial \dot{\xi}_{ \pm}}{\partial \xi}}\left\{\left.\frac{\partial \xi}{\partial a_{k}}\right|_{\ell, i-1}+\left.\frac{\partial \xi}{\partial a_{k}}\right|_{\ell, i}\right\} .
\end{aligned}
$$

Noting that again $\partial \xi /\left.\partial a_{k}\right|_{\ell, i}$ appears on both sides of equation 155 , and collecting terms yields 


$$
\begin{aligned}
& \left.\frac{\partial \xi}{\partial a_{k}}\right|_{\ell, i} \approx\left[1-\frac{\Delta \eta}{2 m} \frac{\overline{\partial \xi_{ \pm}}}{\partial \xi}\right]^{-1}\left\{\left.\frac{\partial \xi}{\partial a_{k}}\right|_{\ell, i-1}\right. \\
& \left.+\frac{\Delta \eta}{m} \frac{\overline{\partial \xi_{ \pm}}}{\partial a_{k}}+\left.\frac{\Delta \eta}{2 m} \frac{\overline{\partial \xi_{ \pm}}}{\partial \xi} \frac{\partial \xi}{\partial a_{k}}\right|_{\ell, i-1}\right\}
\end{aligned}
$$

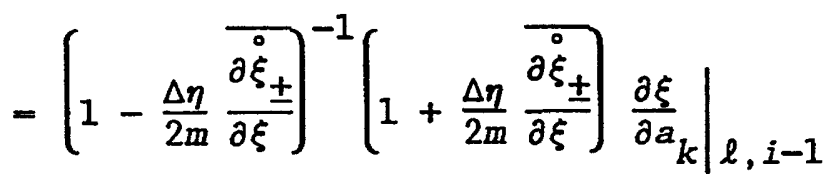

$$
\begin{aligned}
& +\left(1-\frac{\Delta \eta}{2 m} \frac{\partial \dot{\sigma}_{\underline{ \pm}}}{\partial \xi}\right)^{-1} \frac{\Delta \eta}{\frac{\partial \dot{\xi}_{ \pm}}{m}}
\end{aligned}
$$

Define

$$
\begin{aligned}
& \alpha=\left(1-\frac{\Delta \eta}{2 m} \frac{\overline{\partial \dot{\xi}_{ \pm}}}{\partial \xi}\right) \\
& \beta=\left(1+\frac{\Delta \eta}{2 m} \frac{\partial \dot{\delta}_{ \pm}}{\partial \xi}\right) .
\end{aligned}
$$

Then equation 156 can be written

$$
\begin{gathered}
\left.\frac{\partial \xi}{\partial a_{k}}\right|_{\ell, i}=\left.\alpha^{-1} \beta \frac{\partial \xi}{\partial a_{k}}\right|_{\ell, i-1} \\
+\alpha^{-1} \frac{\overline{\Delta \eta}}{\frac{\partial \xi_{ \pm}}{\partial a_{k}}}
\end{gathered}
$$

Sequentially form

$$
\begin{array}{r}
\left.\frac{\partial \xi}{\partial a_{k}}\right|_{\ell, 1},\left.\frac{\partial \xi}{\partial a_{k}}\right|_{\ell, 2}, \cdots \\
\left.\frac{\partial \xi}{\partial a_{k}}\right|_{\ell, m}=\left.\frac{\partial \xi}{\partial a_{k}}\right|_{\ell}
\end{array}
$$

as follows: since $\eta_{\ell, 0}=\eta_{\ell-1}$, we have

$$
\left.\left.\frac{\partial \xi}{\partial a_{k}}\right|_{\ell, 1} \approx \alpha^{-1} \beta \frac{\partial \xi}{\partial a_{k}}\right|_{\ell-1}+\alpha^{-1} \frac{\Delta \eta}{m} \frac{\overline{\partial \xi_{ \pm}}}{\partial a_{k}}
$$

Continuing,

$$
\begin{aligned}
& \left.\left.\frac{\partial \xi}{\partial a_{k}}\right|_{\ell, 2} \approx \alpha^{-1} \beta \frac{\partial \xi}{\partial a_{k}}\right|_{\ell, 1}+\alpha^{-1} \frac{\Delta \eta}{\frac{\partial \xi_{ \pm}}{m}} \\
& =\alpha^{-1} \beta\left\{\left.\alpha^{-1} \beta \frac{\partial \xi}{\partial a_{k}}\right|_{\ell-1}+\alpha^{-1} \frac{\Delta \eta}{\dot{m}} \frac{\overline{\partial \xi_{ \pm}}}{\partial a_{k}}\right\} \\
& +\alpha^{-1} \frac{\Delta \eta}{\bar{m}} \frac{\overline{\partial \xi_{ \pm}}}{\partial a_{k}} \\
& =\left.\alpha^{-2} \beta^{2} \frac{\partial \xi}{\partial a_{k}}\right|_{\ell-1} \\
& +\alpha^{-1}\left[\alpha^{-1} \beta+1\right] \frac{\Delta \eta}{m} \frac{\overline{\partial \xi_{ \pm}}}{\partial a_{k}}
\end{aligned}
$$

Continuing further to $i=m$, we determine

$$
\begin{aligned}
& \left.\left.\frac{\partial \xi}{\partial a_{k}}\right|_{\ell} \approx \alpha^{-m} \beta^{m} \frac{\partial \xi}{\partial a_{k}}\right|_{\ell-1} \\
& +\alpha^{-1}\left[\alpha^{-(m-1)}{ }_{\beta}^{(m-1)}+\alpha^{-(m-2)} \beta^{(m-2)}\right. \\
& \left.+\ldots+\alpha^{-1} \beta+1\right] \frac{\Delta \eta}{m} \frac{\overline{\partial \xi_{ \pm}}}{\partial a_{k}} .
\end{aligned}
$$

Noting that

$$
\sum_{i=0}^{m-1}\left(\alpha^{-1} \beta\right)^{i}=\frac{1-\left(\alpha^{-1} \beta\right)^{m}}{1-\alpha^{-1} \beta}
$$




$$
\begin{aligned}
\left.\frac{\partial \xi}{\partial a_{k}}\right|_{\ell} & =\left.\alpha^{-m} \beta^{m} \frac{\partial \xi}{\partial a_{k}}\right|_{\ell-1} \\
& +\alpha^{-1} \frac{\overline{1-\left(\alpha^{-1} \beta\right)^{m}}}{1-\alpha^{-1} \beta} \frac{\Delta \eta}{m} \frac{\partial \xi_{ \pm}}{\partial a_{k}}
\end{aligned}
$$

Equation 165 is the numerical quadrature used when equation 148 indicates that equation 145 should not be used.

\section{Partial Derivatives for the Continuity Equation}

The partial derivatives with respect to the nodal variables for the continuity integral, equation 81 , are given by

$$
\begin{aligned}
\frac{\partial F_{c}}{\partial a_{k}^{Q}} & =\int_{-1}^{1} \int_{-1}^{1} \frac{\partial}{\partial a_{k}^{Q}}\left(\frac{\partial Q}{\partial x}\right)|\mathrm{J}| d \xi d \eta \\
\frac{\partial F_{c}}{\partial a_{k}^{h}} & =\int_{-1}^{1} \int_{-1}^{1}\left\{\left[\frac{\partial h}{\partial t}\right] \frac{\partial B}{\partial a_{k}^{h}}\right. \\
& \left.+[B] \frac{\partial}{\partial a_{k}^{h}}\left(\frac{\partial h}{\partial t}\right)\right\}|\mathrm{J}| d \xi d \eta
\end{aligned}
$$

The integrals in equations 81,166 , and 167 are approximated numerically by two-point Gaussian quadrature in the local space-time element. If $I_{c}(x, t)$ represents one of the integrands, excluding the determinant $|J|$ of the Jacobian matrix, the Gaussian quadrature is

$$
\begin{aligned}
& \int_{-1}^{1} \int_{-1}^{1} I_{c}(x(\xi, \eta), t(\xi, \eta))|J| d \xi d \eta \\
& \left.\quad \approx 2\left[I_{c}|\mathrm{~J}|\right]\right|_{\xi=-0.57735027, \eta=0} \\
& +\left.2\left[I_{c}|\mathrm{~J}|\right]\right|_{\xi=0.57735027, \eta=0}
\end{aligned}
$$

\section{PARAMETERS}

Channel geometry is assumed to be specified at data cross sections along the length of the river. For each of these sections, channel width, wetted perimeter, and Manning's " $n$ " are specified as functions of elevation. The locations of the data cross sections need not correspond to the locations of the computational cross sections. This independence of the two types of cross sections allows computational cross sections to be located and concentrated where they are most beneficial in describing the dynamics of a particular problem.

For gentle slopes, the choice of a scheme to interpolate channel geometry is easy: linear interpolation will suffice. As the slope steepens, however, solution instabilities may arise from discontinuities in derivatives $\partial / \partial z$ of channel parameters within a cross section, or in derivatives $\partial / \partial x$ along the channel length, as has been noted by Franz (1976), among others. For this reason, Hermitian interpolations are used to provide continuously differentiable approximations of channel parameters.

The Hermitian interpolation is carried out sequentially, first in elevation within cross sections, and then along the channel length. This sequential processing is used because channel geometry is most conveniently specified with variable elevation increments $\Delta z$ between data specifications at elevations $z_{k}$. These elevations are chosen during data collection, depending on the geometry of the particular cross section, and vary from one section to the next. It is, therefore, not convenient to try to match $\Delta z$ segments from one cross section to the next, as would be done in a bi-Hermitian approach in $x \times z$ space.

The sequential interpolation process is as follows. Cross sections that surround the stream coordinate $x$ where the interpolation is desired are first identified. Thus, let $x_{1}$, $x_{2}, \ldots, x_{n}$ denote the stream coordinates at which data are specified. Choose the smallest $x_{j} \geq x_{2}$ so that $x<x_{j}$ or $x_{j}=x_{n}$. The cross sections at $x_{j-1}$ and $x_{j}$ are the nearest neighbors for the desired location $x$, and the cross sections at $x_{j-2}$ and $x_{j+1}$ are the next-nearest neighbors. (If $x_{j}=x_{2}$, then $x_{j-2}$ cannot be formed, and similarly if $x_{j}=x_{n}$, then $x_{j+1}$ does not correspond to a defined cross section. The method to be described in this section will make allowance for these special cases.) At each of these four data cross sections, construct interpolations of channel parameters in water depth $h$. Focusing attention on one of these cross sections, let $z_{1}, z_{2}, \ldots, z_{m}$ be the elevations at the cross section at which channel parameters are specified, where $z_{b}=z_{1}$ is the bottom, or thalweg, elevation of this cross section. Recognizing that water surface elevation $z$ is related to water depth $h$ simply by $z=z_{b}+h$, define depth nodes $h_{k} \equiv z_{k}-z_{b}$ corresponding to the elevations where data are given. In a manner similar to choosing near-neighbors for $x$, choose the smallest $h_{k} \geq h_{2}$ so 
that $h<h_{k}$ or $h_{k}=h_{m}$. The nearest neighbors to $h$ are $h_{k-1}$ and $h_{k}$, and the next-nearest neighbors are $h_{k-2}$ and $h_{k+1}$. Again, one of the next-nearest neighbors will not be defined for the special cases $h_{k}=h_{2}$ and $h_{k}=h_{m}$, and how to deal with these special cases will be treated in the discussion in this section. The Hermitian interpolation is defined on the standard interval $[-1,1]$, with independent variable $\zeta$, using the following linear mapping from global coordinate $h \in\left[h_{k-1}, h_{k}\right]$ to $\zeta$ :

$$
5-\frac{2\left(h-h_{k-1}\right)}{\left(h_{k}-h_{k-1}\right)}-1
$$

implying that

$$
\frac{d \zeta}{d h}=\frac{2}{\left(h_{k}^{-h_{k-1}}\right)}
$$

The Hermitian interpolations in water depth $h$, or, equivalently, elevation $z=h+z_{b}$, at the four neighboring cross sections are specified by

$$
\begin{aligned}
B^{*}\left(x_{j+r}, h\right) & =\psi_{0 m} B^{*}\left(x_{j+r}, h_{k-1}\right) \\
& +\psi_{O p} B^{*}\left(x_{j+r}, h_{k}\right) \\
& +\left.\psi_{1 m} \frac{d h}{d \zeta} \frac{\partial B}{\partial h}\right|_{\left(x_{j+r}, h_{k-1}\right)} \\
& +\left.\psi_{1 p} \frac{d h}{d \zeta} \frac{\partial B}{\partial h}\right|_{\left(x_{j+r}, h_{k}\right)}
\end{aligned}
$$

where $r=-2,-1,0$, or 1 as appropriate, to indicate the cross section being considered. There are similar expressions for $P^{*}$ and $n^{*}$. (See equations 108, 109, and 110.) (The $m$ and the $p$ in the subscripts mnemonically stand for "minus" and "plus".) The basis functions $\psi_{1 m}$ and $\psi_{1 p}$ multiply nodal derivatives $\partial / \partial \zeta$ in the local one-dimensional element, and thus require the factor $d h / d \zeta$ to convert from global partial derivatives with respect to $h$ to local partial derivatives with respect to $\zeta$. The Hermitian basis functions are given by

$$
\begin{aligned}
& \psi_{0 m}=(5-1)^{2}(5+2) / 4 \\
& \psi_{0 p}=(5+1)^{2}(-5+2) / 4 \\
& \psi_{1 m}=(5-1)^{2}(5+1) / 4
\end{aligned}
$$

$$
\psi_{1 p}=(5+1)^{2}(5-1) / 4 \text {. }
$$

In some cases, extrapolation above the highest defined data depth $h_{m}$ may be required. Extrapolation by equation 171 using equations 172 to 175 directly is often not satisfactory in practice; rather, define an extrapolation based on the subinterval $\left[h_{m-1}, h_{m}\right]$ to be linear, and continuously differentiable at $h=h_{m}$, as follows:

If $\zeta>1$, then

$$
\begin{aligned}
& \psi_{0 m}=0 \\
& \psi_{0 p}=1 \\
& \psi_{1 m}=0 \\
& \psi_{1 p}=5-1
\end{aligned}
$$

The definitions of $\psi$ in equations 172 to 179 , used for $h$-interpolation, will also be used to give similar definitions for $x$-interpolation. For depths, only extrapolation above the highest specified depth can be contemplated. However, extrapolations beyond both the first and last cross sections of the reach are possible in the $x$-direction, although such extrapolations are rarely needed. Therefore, to allow reference for $x$-extrapolation beyond the first cross section, the lower-margin extrapolation using the depth notation $\psi$ is included for completeness. Define an extrapolation that is based on the first subinterval $\left[h_{1}, h_{2}\right]$ to be linear, and continuously differentiable at $h=h_{1}$, as follows:

If $\zeta<-1$, then

$$
\begin{aligned}
& \psi_{0 m}=1 \\
& \psi_{0 p}=0 \\
& \psi_{1 m}=\zeta+1 \\
& \psi_{1 p}=0 .
\end{aligned}
$$

To calculate the cross-sectional area below the water surface, form the integral

$$
A^{*}\left(x_{j+r}, h\right)=\int_{0}^{h} B^{*}\left(x_{j+r}, \hat{h}\right) d \hat{h}
$$

Sum the latter integral over the depth nodes $h_{k}$ at which data are specified: 


$$
\begin{aligned}
A^{*}\left(x_{j+r}, h\right) & =\sum_{\gamma=2}^{k-1} \int_{h \gamma-1}^{h} B^{*}\left(x_{j+r}, \hat{h}\right) \hat{d h} \\
& +\int_{h=1}^{h} B^{*}\left(x_{j+r}, \hat{h}\right) d \hat{h} .
\end{aligned}
$$

Note that the last integral in equation 185 involves the selected interpolating interval $h \in\left[h_{k-1}, h_{k}\right]$. This integral can be written in terms of the Hermitian expansion of the top width $B^{*}$, using equation 171 , as

$$
\begin{aligned}
& \int_{h k-1}^{h} B^{*}\left(x_{j+r}, \hat{h}\right) d \hat{h} \\
& =\int_{-1}^{\zeta}\left(\psi_{0 m} B^{*}\left(x_{j+r}, h_{k-1}\right)+\psi_{0 p} B^{*}\left(x_{j+r}, h_{k}\right)\right. \\
& +\left.\psi_{1 m} \frac{d h}{d \zeta} \frac{\partial B^{*}}{\partial h}\right|_{\left(x_{j+r}, h_{k-1}\right)} \\
& \left.+\left.\psi_{1 p} \frac{d h}{d \zeta} \frac{\partial B^{*}}{\partial h}\right|_{\left(x_{j+r}, h_{k}\right)}\right)_{\frac{d h}{d \zeta}}^{d \zeta} \\
& =\int_{-1}^{\zeta} \psi_{0 m} \hat{d \zeta} \frac{d h}{d \zeta} B^{*}\left(x_{j+r}, h_{k-1}\right) \\
& +\int_{-1}^{\zeta} \psi_{0 p} \hat{d \zeta} \frac{d h}{d \zeta} B^{*}\left(x_{j+r}, h_{k}\right) \\
& +\left.\int_{-1}^{\zeta} \psi_{1 m} \hat{d \zeta}\left(\frac{d h}{d \zeta}\right)^{2} \frac{\partial B^{*}}{\partial h}\right|_{\left(x_{j+r}, h_{k-1}\right)} \\
& +\left.\int_{-1}^{\zeta} \psi_{1 p} \hat{d \zeta}\left(\frac{d h}{d \zeta}\right)^{2} \frac{\partial B^{*}}{\partial h}\right|_{\left(x_{j+r}, h_{k}\right)} \text {. }
\end{aligned}
$$

The last four integrals of the Hermitian basis functions in equations 186 can easily be calculated from the basis function definitions, equations 172 to 175 :

$$
\int_{-1}^{\zeta} \psi_{1 m} d \hat{\zeta}=\left(3 \zeta^{4}-4 \zeta^{3}-6 \zeta^{2}+12 \zeta+11\right) / 48
$$

$$
\int_{-1}^{\zeta} \psi_{1 p} d \hat{\zeta}=\left(3 \zeta^{4}+4 \zeta^{3}-6 \zeta^{2}-12 \zeta-5\right) / 48
$$

For the special case of extrapolation to $h>h_{m}$, the integrals are as follows:

If $\zeta>1$, then

$$
\begin{aligned}
\int_{-1}^{\zeta} \psi_{0 m} d \hat{\zeta}-1 \\
\int_{-1}^{\zeta} \psi_{0 p} d \hat{\zeta}=\zeta \\
\int_{-1}^{\zeta} \psi_{1 p}^{d \hat{\zeta}}=\int_{-1}^{\zeta} \psi_{1 p} \psi_{1 m} d \hat{d \zeta}-\frac{1}{3} \\
=-\frac{1}{3}+\int_{1}^{\zeta} \psi_{1 p} d \hat{\zeta} \\
=\left(3 \zeta^{2}-6 \zeta+1\right) d \hat{\zeta}
\end{aligned}
$$


The $-1 / 3$ in equation 194 comes from the evaluation of equation 190 for $\zeta=1$. For the special case of extrapolation to $h<h_{1}$, the integrals are as follows:

If $\zeta<-1$, then

$$
\begin{aligned}
\int_{-1}^{\zeta} \psi_{0 p} d \hat{\zeta} & =\int_{-1}^{\zeta} \psi_{1 p} \hat{d \zeta}=0 \\
\int_{-1}^{\zeta} \psi_{0 m} \hat{d \zeta} & =\zeta+1 \\
\int_{-1}^{\zeta} \psi_{1 m} \hat{d \zeta} & =\int_{-1}^{\zeta} \hat{(\zeta+1) d \hat{\zeta}} \\
& =\left(\zeta^{2}+2 \zeta+1\right) / 2 .
\end{aligned}
$$

In equation 185 , the integrals within the summation are similar to the last integral in the equation, except that the integration is the complete depth range of lower elements $h \in\left[h_{\gamma-1}, h_{\gamma}\right], \gamma=2, \ldots, k-1$, in the cross section. These are computed by use of an expression similar to equation 186, but with $\zeta=1$, and with $[\gamma-1, \gamma]$ for $\gamma=2, \ldots, k-1$ replacing $[k-1, k]$. These integrals can be calculated at the beginning of the modeling for the complete set of depths at each cross section and stored for later use in equation 185 .

Partial derivatives of the channel parameters with respect to depth are also needed. At the cross sections $x_{j-2}$, $x_{j-1}, x_{j}$, and $x_{j+1}$, the derivatives are obtained by differentiating equation 171 to yield

$$
\begin{gathered}
\left.\frac{\partial B^{*}}{\partial h}\right|_{\left(x_{j+r}, h\right)}=\frac{\partial \psi_{0 m}}{\partial \zeta} \frac{d \zeta}{d h} B^{*}\left(x_{j+r}, h_{k-1}\right) \\
+\frac{\partial \psi_{0 p}}{\partial \zeta} \frac{d \zeta}{d h} B^{*}\left(x_{j+r}, h_{k}\right) \\
+\left.\frac{\partial \psi_{1 m}}{\partial \zeta} \frac{\partial B}{\partial h}\right|_{\left(x_{j+r}, h_{k-1}\right)} \\
+\left.\frac{\partial \psi_{1 p}}{\partial \zeta} \frac{\partial B}{\partial h}\right|_{\left(x_{j+r}, h_{k}\right)}
\end{gathered}
$$

with similar expressions for $P^{*}$ and $n^{*}$. (The partial derivatives with respect to $h$ evaluated at an arbitrary point $(x, h)$ will be derived after the $x$-interpolation is discussed.) In the last two terms of equation 198, a factor $d \zeta / d h$ has cancelled a factor $d h / d \zeta$ of equation 171. The derivatives of the Hermitian basis functions that appear in equation 198 are given by

$$
\begin{aligned}
& \frac{\partial \psi_{0 m}}{\partial \zeta}=3(\zeta-1)(\zeta+1) / 4 \\
& \frac{\partial \psi_{0 P}}{\partial \zeta}=3(\zeta+1)(-\zeta+1) / 4 \\
& \frac{\partial \psi_{1 m}}{\partial \zeta}=(\zeta-1)(3 \zeta+1) / 4 \\
& \frac{\partial \psi_{1 p}}{\partial \zeta}=(\zeta+1)(3 \zeta-1) / 4
\end{aligned}
$$

For the special case of extrapolation to $h>h_{m}$, the derivatives are as follows:

If $\zeta>1$, then

$$
\begin{gathered}
\frac{\partial \psi_{0 m}}{\partial \zeta}=\frac{\partial \psi_{0 p}}{\partial \zeta}=\frac{\partial \psi_{1 m}}{\partial \zeta}=0 \\
\frac{\partial \psi_{1 p}}{\partial \zeta}=1 .
\end{gathered}
$$

For the special case of extrapolation to $h<h_{1}$, the derivatives are as follows:

If $\zeta<-1$, then

$$
\begin{gathered}
\frac{\partial \psi_{0 m}}{\partial \zeta}=\frac{\partial \psi_{0 p}}{\partial \zeta}=\frac{\partial \psi_{1 p}}{\partial \zeta}=0 \\
\frac{\partial \psi_{1 m}}{\partial \zeta}=1 .
\end{gathered}
$$

The partial derivatives of the channel parameters with respect to $h$ at the nodes $\zeta= \pm 1$ of the element appear in the expansions of equations 171, 186, and 198, and are approximated as

$$
\begin{gathered}
\left.\frac{\partial B^{*}}{\partial h}\right|_{\left(x_{j+r}, h_{k-1}\right)}=\left[B^{*}\left(x_{j+r}, h_{k}\right)\right. \\
\left.-B^{*}\left(x_{j+r}, h_{k-\rho}\right)\right] /\left(h_{k}-h_{k-\rho}\right)
\end{gathered}
$$




$$
\begin{array}{r}
\left.\frac{\partial B^{*}}{\partial h}\right|_{\left(x_{j+r}, h_{k}\right)}=\left[B^{*}\left(x_{j+r}, h_{k+\sigma}\right)\right. \\
\left.-B^{*}\left(x_{j+r}, h_{k-1}\right)\right] /\left(h_{k+\sigma}-h_{k-1}\right),
\end{array}
$$

with similar expressions for $P^{*}$ and $n^{*}$. In the most common situation that occurs when $k \neq 2$, set subscript increment $\rho=2$. To treat the special case of $k=2$, that is, the lowest element in the cross section, use $\rho=1$. Similarly, for the usual case when $k \neq m$, set $\sigma=1$. In the special case when $k=m$ at the uppermost defined elevation of the cross section, set $\sigma=0$.

Note that $B^{*}\left(x_{j+r}, h\right), P^{*}\left(x_{j+r}, h\right), n^{*}\left(x_{j+r}, h\right)$,

$\left.\frac{\partial B^{*}}{\partial h}\right|_{\left(x_{j+r}, h\right)},\left.\frac{\partial P^{*}}{\partial h}\right|_{\left(x_{j+r}, h\right)}$, and $\left.\frac{\partial n^{*}}{\partial h}\right|_{\left(x_{j+r}, h\right)}$, as given by equations 171 and 198, are continuous functions of $h$. This is true even across element boundaries $h_{k}$, because $\left.\frac{\partial B^{*}}{\partial h}\right|_{\left(x_{j+r}, h_{k-1}\right)}$ and $\left.\frac{\partial B^{*}}{\partial h}\right|_{\left(x_{j+r}, h_{k}\right)}$ are defined the same way on both neighboring elements, and although $d \zeta / d h$ is discontinuous across boundaries, $\psi_{1 m}, \psi_{1 p}, \partial \psi_{0 m} / \partial \zeta$, and $\partial \psi_{0 p} / \partial \zeta$ are all zero at $\zeta= \pm 1$. Note also that $A^{*}\left(x_{j+r}, h\right)$ and $\left.\frac{\partial A^{*}}{\partial h}\right|_{\left(x_{j+r}, h\right)}=B^{*}\left(x_{j+r}, h\right)$, defined by equation 184 , are continuous functions of $h$.

Having defined interpolated values of the channel parameters at water depth $h$, at the neighboring and nearneighboring cross sections $x_{j-2}, x_{j-1}, x_{j}$, and $x_{j+1}$, we now proceed to the Hermitian interpolation in river coordinate $x$. The Hermitian expansion is given by

$$
\begin{aligned}
B^{*}(x, h) & =\phi_{0 m} B^{*}\left(x_{j-1}, h\right)+\phi_{0 p} B^{*}\left(x_{j}, h\right) \\
& +\left.\phi_{1 m} \frac{d x}{d \Xi} \frac{\partial B}{\partial x}\right|_{\left(x_{j-1}, h\right)} \\
& +\left.\phi_{1 p} \frac{d x}{d \Xi} \frac{\partial B}{\partial x}\right|_{\left(x_{j}, h\right)}
\end{aligned}
$$

with similar expressions for $A^{*}, P^{*}$, and $n^{*}$. Channel bottom elevation $z_{b}$ at river coordinate $x$ is interpolated in the same manner, but there is no dependence on water depth $h$. The Hermitian basis functions $\phi_{0 m}, \phi_{0 p}, \phi_{1 m}$, and $\phi_{1 p}$ are defined in local element $\Xi \in[-1,1]$ corresponding to global element $x \in\left[x_{j-1}, x_{j}\right]$. (We use capital Greek letter xi, $\Xi$, instead of the more typically used lower case $x i, \xi$, to distinguish the interpolation of channel geometry being defined here from the local computational space-time element of equations 22 and 23 that was defined earlier.) The linear transformation between $\Xi$ and $x$ is similar to the mapping between $\zeta$ and $z$ :

$$
\Xi=\frac{2\left(x-x_{j-1}\right)}{\left(x_{j}-x_{j-1}\right)}-1
$$

implying that

$$
\frac{d E}{d x}=\frac{2}{\left(x_{j}-x_{j-1}\right)} .
$$

The basis functions themselves are defined by replacing $\psi$ with $\phi$ and $\zeta$ with $\Xi$ in equations 172 to 183 .

Partial derivatives with respect to $x$ are given by

$$
\begin{aligned}
& \left.\frac{\partial B^{*}}{\partial \mathrm{x}}\right|_{(\mathrm{x}, h)}=\frac{\partial \phi_{0 m}}{\partial \Xi} \frac{d \Xi}{d \mathrm{x}} B^{*}\left(\mathrm{x}_{j-1}, h\right) \\
& +\frac{\partial \phi_{0 P}}{\partial \Xi} \frac{d \Xi}{d x} B^{*}\left(\mathrm{x}_{j}, h\right)+\left.\frac{\partial \phi_{1 m}}{\partial \Xi} \frac{\partial B}{\partial \mathrm{x}}\right|_{\left(x_{j-1}, h\right)} \\
& +\left.\frac{\partial \phi_{1 P}}{\partial \Xi} \frac{\partial B}{\partial \mathrm{x}}\right|_{\left(\mathrm{x}_{j}, h\right)},
\end{aligned}
$$

with similar expressions for partial derivatives with respect to $x$ of $A^{*}, P^{*}, n^{*}$, and $z_{b}$. In equation 212 , the partial derivatives of the Hermitian basis functions with respect to $\Xi$ are obtained by replacing $\psi$ with $\phi$ and $\zeta$ with $\Xi$ in equations 199 to 206.

In equations 209 and 212 , the nodal values at $\Xi= \pm 1$ of the partial derivatives with respect to $x$ are approximated by

$$
\begin{array}{r}
\left.\frac{\partial B^{*}}{\partial x}\right|_{\left(x_{j-1}, h\right)}=\left[B^{*}\left(x_{j}, h\right)\right. \\
\left.-B^{*}\left(x_{j-\lambda}, h\right)\right] /\left(x_{j}-x_{j-\lambda}\right)
\end{array}
$$

$$
\begin{aligned}
\left.\frac{\partial B^{*}}{\partial x}\right|_{\left(x_{j}, h\right)}= & {\left[B^{*}\left(x_{j+\nu}, h\right)\right.} \\
& \left.-B^{*}\left(x_{j-1}, h\right)\right] /\left(x_{j+\nu}-x_{j-1}\right),
\end{aligned}
$$

with similar expressions for $A^{*}, P^{*}, n^{*}$, and $z_{b}$. In the most common situation when $j \neq 2$, set subscript increment $\lambda=2$. For the special case $j=2$, that is, the first element in the 
longitudinal profile, use $\lambda=1$. Similarly, for the usual case when $j \neq n$, set $v=1$. In the special case when $j=n$ at the last element in the longitudinal profile, set $\mathrm{v}=0$.

The functions $B^{*}, A^{*}, P^{*}, n^{*}, \partial B^{*} / \partial x, \partial A^{*} / \partial x$, $\partial P^{*} / \partial x$, and $\partial n^{*} / \partial x$ defined by equations 209 and 212 are continuous functions of $x$ and $h$, and $z_{b}$ and $\partial z_{b} / \partial x$ are continuous functions of $x$. This holds true even across element boundaries $x_{j}$, even though $d \Xi / d x$ may be discontinuous there, because $\phi_{1 m}, \phi_{1 p}, \partial \phi_{0 m} / \partial \Xi$ and $\partial \phi_{0 p} / \partial \Xi$ are zero at $\Xi= \pm 1$, and because the nodal values of the partial derivatives with respect to $x$ are defined the same in both neighboring elements.

We now derive an expression for the partial derivatives of channel parameters with respect to $h$ at any given point $(x, h)$, rather than only at cross sections, to which equation 198 is restricted. We show that the partial derivative of equation 209 with respect to $h$ is consistent with the Hermitian expansion of cross-sectional values of $\partial B^{*} / \partial h$ at $x_{j-2}, x_{j-1}, x_{j}$, and $x_{j+1}$ using the same equation 209. Taking the partial derivative of equation 209 with respect to $h$ yields

$$
\begin{aligned}
& \left.\frac{\partial B^{*}}{\partial h}\right|_{(x, h)}=\left.\phi_{0 m} \frac{\partial B^{*}}{\partial h}\right|_{\left(x_{j-1}, h\right)} \\
& +\left.\phi_{0 p} \frac{\partial B^{*}}{\partial h}\right|_{\left(x_{j}, h\right)} \\
& +\phi_{1 m} \frac{d x}{d \Xi} \frac{\partial}{\partial h}\left(\left.\frac{\partial B^{*}}{\partial x}\right|_{\left(x_{j-1}, h\right)}\right) \\
& +\phi_{1 p} \frac{d x}{d \Xi} \frac{\partial}{\partial h}\left(\left.\frac{\partial B^{*}}{\partial x}\right|_{\left(x_{j}, h\right)}\right) \\
& =\left.\phi_{0 m} \frac{\partial B^{*}}{\partial h}\right|_{\left(x_{j-1}, h\right)}+\left.\phi_{0 p} \frac{\partial B^{*}}{\partial h}\right|_{\left(x_{j}, h\right)} \\
& +\phi_{1 m} \frac{d x}{d \Xi} \frac{\partial}{\partial h}\left(B^{*}\left(x_{j}, h\right)\right. \\
& \left.-B^{*}\left(x_{j-\lambda}, h\right)\right) /\left(x_{j}-x_{j-\lambda}\right) \\
& +\phi_{1 p} \frac{d x}{d \Xi} \frac{\partial}{\partial h}\left[B^{*}\left(x_{j+\nu}, h\right)\right. \\
& \left.-B^{*}\left(x_{j-1}, h\right)\right) /\left(x_{j+\nu}-x_{j-1}\right)
\end{aligned}
$$

$$
\begin{aligned}
& =\left.\phi_{0 m} \frac{\partial B^{*}}{\partial h}\right|_{\left(x_{j-1}, h\right)}+\left.\phi_{0 p} \frac{\partial B^{*}}{\partial h}\right|_{\left(x_{j}, h\right)} \\
& +\phi_{1 m} \frac{d x}{d \Xi}\left(\left.\frac{\partial B^{*}}{\partial h}\right|_{\left(x_{j}, h\right)}\right. \\
& \left.-\left.\frac{\partial B^{*}}{\partial h}\right|_{\left(x_{j-\lambda}, h\right)}\right) /\left(x_{j}-x_{j-\lambda}\right) \\
& +\phi_{1 p} \frac{d x}{d \Xi}\left[\left.\frac{\partial B^{*}}{\partial h}\right|_{\left(x_{j+\nu}, h\right)}\right. \\
& \left.-\left.\frac{\partial B^{*}}{\partial h}\right|_{\left(x_{j-1}, h\right)}\right) /\left(x_{j+\nu}-x_{j-1}\right) \\
& =\left.\phi_{0 m} \frac{\partial B^{*}}{\partial h}\right|_{\left(x_{j-1}, h\right)}+\left.\phi_{0 p} \frac{\partial B^{*}}{\partial h}\right|_{\left(x_{j}, h\right)} \\
& +\left.\phi_{1 m} \frac{d x}{d \Xi} \frac{\partial}{\partial x}\left(\frac{\partial B^{*}}{\partial h}\right)\right|_{\left(x_{j-1}, h\right)} \\
& +\left.\phi_{1 p} \frac{d x}{d \Xi} \frac{\partial}{\partial x}\left(\frac{\partial B^{*}}{\partial h}\right)\right|_{\left(x_{j}, h\right)} .
\end{aligned}
$$

The expression to the right of the last equality in equation 215 is the $x$-interpolation of cross-sectional values of $\partial B^{*} / \partial h$. The derivation of equation 215 also applies to the partial derivative with respect to $h$ of $P^{*}$ and $n^{*}$.

The definition of cross-sectional area $A^{*}(x, h)$, obtained using the interpolation of equation 209 and the nodal derivative approximations of equations 213 and 214 (and using $A^{*}$ in place of $B^{*}$ in each equation), is consistent with the integration of width over depth done at coordinate $x$ :

$$
A^{*}(x, h)=\int_{0}^{h} B^{*}(x, \hat{h}) d \hat{h} .
$$

Proof of equation 216: Expanding the right side of equation 216, we have

$\int_{0}^{h} B^{*}(x, \hat{h}) d \hat{h}$ 


$$
\begin{aligned}
& =\int_{0}^{h} \phi_{0 m} B^{*}\left(x_{j-1}, \hat{h}\right)+\phi_{0 p} B^{*}\left(x_{j}, \hat{h}\right) \\
& +\left.\phi_{1 m} \frac{d x}{d \Xi} \frac{\partial B^{*}}{\partial x}\right|_{\left(x_{j-1}, \hat{h}\right)} \\
& \left.+\left.\phi_{1 p} \frac{d x}{d \Xi} \frac{\partial B^{*}}{\partial x}\right|_{\left(x_{j}, \hat{h}\right)}\right) \hat{d h} \\
& =\phi_{0 m} \int_{0}^{h} B^{*}\left(x_{j-1}, \hat{h}\right) \hat{h}+\phi_{0 p} \int_{0}^{h} B^{*}\left(x_{j}, \hat{h}\right) \hat{d h} \\
& +\left.\phi_{1 m} \frac{d x}{d \Xi} \int_{0}^{h} \frac{\partial B^{*}}{\partial x}\right|_{\left(x_{j-1}, \hat{h}\right)} \hat{d h} \\
& +\left.\phi_{1 p} \frac{d x}{d \Xi} \int_{0}^{h} \frac{\partial B}{\partial x}\right|_{\left(x_{j}, \hat{h}\right)} d \hat{h} \\
& =\phi_{0 m} A^{*}\left(x_{j-1}, h\right)+\phi_{0 p} A^{*}\left(x_{j}, h\right) \\
& +\phi_{1 m} \frac{d x}{d \Xi} \int_{0}^{h} \int\left[B^{*}\left(x_{j}, \hat{h}\right)\right. \\
& \left.\left.-B^{*}\left(x_{j-\lambda}, \hat{h}\right)\right] /\left(x_{j}-x_{j-\lambda}\right)\right] d \hat{h} \\
& +\phi_{1 p} \frac{d \mathrm{x}}{d \Xi} \int_{0}^{h} \int\left[B^{*}\left(\mathrm{x}_{j+\nu}, \hat{h}\right)\right. \\
& \left.\left.-B^{*}\left(x_{j-1}, \hat{h}\right)\right] /\left(x_{j+\nu}-x_{j-1}\right)\right) d \hat{h} \\
& =\phi_{0 m} A^{*}\left(\mathrm{x}_{j-1}, h\right)+\phi_{0 p} A^{*}\left(\mathrm{x}_{j}, h\right) \\
& +\phi_{1 m} \frac{d x}{d \Xi}\left\{\int_{0}^{h} B^{*}\left(x_{j}, \hat{h}\right) d \hat{h}\right. \\
& \left.-\int_{0}^{h} B^{*}\left(x_{j-\lambda}, \hat{h}\right) d \hat{h}\right\} /\left(x_{j}-x_{j-\lambda}\right) \\
& +\phi_{1 p} \frac{d x}{d \Xi}\left\{\int_{0}^{h} B^{*}\left(x_{j+\nu}, \hat{h}\right) d \hat{h}\right.
\end{aligned}
$$

$$
\begin{aligned}
& \left.-\int_{0}^{h} B^{*}\left(x_{j-1}, \hat{h}\right) d \hat{h}\right\} /\left(x_{j+\nu}-x_{j-1}\right) \\
& =\phi_{0 m} A^{*}\left(x_{j-1}, h\right)+\phi_{0 p} A^{*}\left(x_{j}, h\right) \\
& +\phi_{1 m} \frac{d x}{d \Xi}\left[A^{*}\left(x_{j}, h\right)\right. \\
& \left.-A^{*}\left(x_{j-\lambda}, h\right)\right] /\left(x_{j}-x_{j-\lambda}\right) \\
& +\phi_{1 p} \frac{d x}{d \Xi}\left[A^{*}\left(x_{j+\nu}, h\right)\right. \\
& \left.-A^{*}\left(x_{j-1}, h\right)\right] /\left(x_{j+\nu}-x_{j-1}\right) \\
& =\phi_{0 m} A^{*}\left(x_{j-1}, h\right)+\phi_{0 p} A^{*}\left(x_{j}, h\right) \\
& +\left.\phi_{1 m} \frac{d x}{d \Xi} \frac{\partial A^{*}}{\partial x}\right|_{\left(x_{j-1}, h\right)} \\
& +\left.\phi_{1 p} \frac{d x}{d \Xi} \frac{\partial A^{*}}{\partial x}\right|_{\left(x_{j}, h\right)} .
\end{aligned}
$$

This completes the proof of equation 216. From equation 216 it follows directly that

$$
\frac{\partial A^{*}}{\partial h}=B^{*}
$$

The order of taking partial derivatives of channel parameters with respect to $x$ and $h$ can be interchanged:

$$
\frac{\partial}{\partial h}\left(\frac{\partial B^{*}}{\partial x}\right)=\frac{\partial}{\partial x}\left(\frac{\partial B^{*}}{\partial h}\right) .
$$

Proof of equation 219: Taking the partial derivatives with respect to $x$ of the expansion of $\partial B^{*} / \partial h$ from equation 215 and applying equations 213 and 214 to $\partial B^{*} / \partial h$ instead of $B^{*}$, we have

$$
\begin{aligned}
& \left.\frac{\partial}{\partial x}\left(\frac{\partial B^{*}}{\partial h}\right)\right|_{(x, h)} \\
& =\left.\frac{\partial \phi_{0 m}}{\partial \Xi} \frac{d \Xi}{d x} \frac{\partial B^{*}}{\partial h}\right|_{\left(x_{j-1}, h\right)}
\end{aligned}
$$

Interpolation of Channel Parameters

25 


$$
\begin{aligned}
& +\left.\frac{\partial \phi_{0 P}}{\partial \Xi} \frac{d \Xi}{d x} \frac{\partial B^{*}}{\partial h}\right|_{\left(x_{j}, h\right)} \\
& +\left.\frac{\partial \phi}{\partial \Xi} \frac{\partial}{\partial x}\left(\frac{\partial B^{*}}{\partial h}\right)\right|_{\left(x_{j-1}, h\right)} \\
& +\left.\frac{\partial \phi_{1 P}}{\partial \Xi} \frac{\partial}{\partial x}\left(\frac{\partial B^{*}}{\partial h}\right)\right|_{\left(x_{j}, h\right)} \\
& =\left.\frac{\partial \phi_{0 m}}{\partial \Xi} \frac{d \Xi}{d x} \frac{\partial B^{*}}{\partial h}\right|_{\left(x_{j-1}, h\right)} \\
& +\left.\frac{\partial \phi_{O P}}{\partial \Xi} \frac{d \Xi}{d \mathbf{x}} \frac{\partial B^{*}}{\partial h}\right|_{\left(x_{j}, h\right)} \\
& +\frac{\partial \phi_{1 m}}{\partial \Xi}\left[\left.\frac{\partial B^{*}}{\partial h}\right|_{\left(x_{j}, h\right)}\right. \\
& \left.-\left.\frac{\partial B^{*}}{\partial h}\right|_{\left(x_{j-\lambda}, h\right)}\right) /\left(x_{j}-x_{j-\lambda}\right) \\
& +\frac{\partial \phi_{1 P}}{\partial \Xi}\left(\left.\frac{\partial B^{*}}{\partial h}\right|_{\left(x_{j+\nu}, h\right)}\right. \\
& \left.-\left.\frac{\partial B^{*}}{\partial h}\right|_{\left(x_{j-1}, h\right)}\right) /\left(x_{j+\nu}-x_{j-1}\right) \\
& =\left.\frac{\partial \phi_{0 m}}{\partial \Xi} \frac{d \Xi}{d x} \frac{\partial B^{*}}{\partial h}\right|_{\left(x_{j-1}, h\right)} \\
& +\left.\frac{\partial \phi_{0 P}}{\partial \Xi} \frac{d \Xi}{d x} \frac{\partial B^{*}}{\partial h}\right|_{\left(x_{j}, h\right)} \\
& +\frac{\partial \phi_{1 m}}{\partial \Xi} \frac{\partial}{\partial h} \int\left[B^{*}\left(x_{j}, h\right)\right. \\
& \left.\left.-B^{*}\left(x_{j-\lambda}, h\right)\right] /\left(x_{j}-x_{j-\lambda}\right)\right)
\end{aligned}
$$$$
\begin{aligned}
& +\frac{\partial \phi_{1 P}}{\partial \Xi} \frac{\partial}{\partial h}\left[\left[B^{*}\left(x_{j+\nu}, h\right)\right.\right. \\
& \left.\left.\quad-B^{*}\left(x_{j-1}, h\right)\right] /\left(x_{j+\nu}-x_{j-1}\right)\right)
\end{aligned}
$$$$
=\left.\frac{\partial \phi_{0 m}}{\partial \Xi} \frac{d \Xi}{d x} \frac{\partial B^{*}}{\partial h}\right|_{\left(x_{j-1}, h\right)}
$$$$
+\left.\frac{\partial \phi_{0 P}}{\partial \Xi} \frac{d \Xi}{d x} \frac{\partial B^{*}}{\partial h}\right|_{\left(x_{j}, h\right)}
$$$$
+\frac{\partial \phi_{1 m}}{\partial \Xi} \frac{\partial}{\partial h}\left(\left.\frac{\partial B^{*}}{\partial x}\right|_{\left(x_{j-1}, h\right)}\right)
$$$$
+\frac{\partial \phi_{1 p}}{\partial \Xi} \frac{\partial}{\partial h}\left(\left.\frac{\partial B^{*}}{\partial \mathbf{x}}\right|_{\left(\mathbf{x}_{j}, h\right)}\right)
$$$$
=\left.\frac{\partial}{\partial h}\left(\frac{\partial B^{*}}{\partial x}\right)\right|_{(x, h)} \text {. }
$$

The last equality follows by equation 212 , and the next to the last by equations 213 and 214 , applied this time directly to $B^{*}$. This completes the proof of equation 219 . By similar arguments, the order of differentiation can also be interchanged for the other channel parameters $A^{*}, P^{*}$, and $n^{*}$.

Second partial derivatives of the channel parameters with respect to river coordinate $x$ are obtained by differentiating equation 212 , to yield

$$
\begin{gathered}
\left.\frac{\partial^{2} B^{*}}{\partial x^{2}}\right|_{(x, h)}=\frac{\partial^{2} \phi_{0 m}}{\partial \Xi^{2}}\left(\frac{d \Xi}{d x}\right)_{B}^{2}\left(x_{j-1}, h\right) \\
+\frac{\partial^{2} \phi_{0 P}}{\partial \Xi^{2}}\left[\frac{d \Xi}{d x}\right)^{2} B^{*}\left(x_{j}, h\right) \\
+\left.\frac{\partial^{2} \phi_{1 m}}{\partial \Xi^{2}} \frac{d \Xi}{d x} \frac{\partial B^{*}}{\partial x}\right|_{\left(x_{j-1}, h\right)} \\
+\left.\frac{\partial^{2} \phi_{1 P}}{\partial \Xi^{2}} \frac{d \Xi}{d x} \frac{\partial B}{\partial x}\right|_{\left(x_{j}, h\right)}
\end{gathered}
$$


with similar expressions for the other channel parameters $A^{*}, P^{*}, n^{*}$, and $z_{b}$. In equation 221 , the second partial derivatives of the Hermitian basis functions are obtained by replacing $\psi$ with $\phi$ and $\zeta$ with $\Xi$ in equations 199 to 202 , and then differentiating to obtain

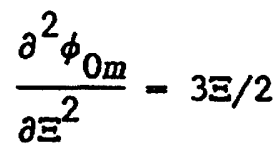

$\frac{\partial^{2} \phi_{0 p}}{\partial \Xi^{2}}=-3 \Xi / 2$
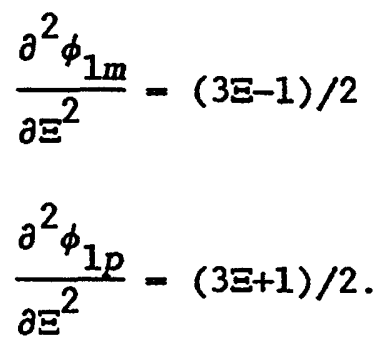

A similar substitution and differentiation of equations 203 to 206 shows that all second partial derivatives of the Hermitian basis functions are zero in the special cases of extrapolation beyond the first or last elements.

\section{STEEP-SLOPE FACTOR}

The factor $\Phi$ in equation 92 adjusts the friction slope determined by Manning's equation (eq. 93) for steep slopes (Jarrett, 1984; Glass and others, 1987). The use of this factor is by no means necessary for the proper performance of the model. The factor is nevertheless included to provide an estimate of friction slope that is, by Jarrett's recent work, perhaps better suited to steep rivers. When the model is run, this factor can be set to unity, if desired. Factor $\Phi$ is derived from equation 9 on page 1,532 of Jarrett's paper, which gives Manning's coefficient " $n$ " as a function of slope $S$ and hydraulic radius $R=A / P$ :

$$
n_{J}=0.39 s^{0.38} R^{-0.16}
$$

where $n_{J}$ is used to distinguish Manning's " $n$ " computed using Jarrett's formula. Equation 226 was derived on the basis of data including slopes ranging from 0.002 to 0.04 , and for hydraulic radii from 0.5 to 7 feet. Although the multiple regression used to derive the coefficients in equation 226 used $S$ defined as friction slope, the sentence after equation 9 in Jarrett's paper indicates that watersurface slope $S_{w}$ could be used instead, at least for fairly uniform channels. Water-surface slope is used here, in this approximate implementation of Jarrett's ideas, and equation 226 is normalized to $S_{0}=0.002$ and $R_{0}=7$ feet, to yield

$$
\begin{aligned}
n_{J} & =\frac{(0.002)^{0.38}(0.39)}{7^{0.16}}\left(\frac{R_{0}}{R}\right)^{0.16}\left(\frac{S_{w}}{S_{0}}\right)^{0.38} \\
& =\left\{0.027\left(\frac{R_{0}}{R}\right)^{0.16}\right\}\left(\frac{S_{w}}{S_{0}}\right)^{0.38}
\end{aligned}
$$

The purpose here is to implement a steep-slope correction to Manning's coefficients specified by more traditional estimates of channel roughness. The data specification given in the previous section already allows $n$ to be a function of water depth, which might conflict with or duplicate the factor in equation 227 that is dependent on hydraulic radius. The approximation used here is to replace the first factor in braces by $n$, the input, traditional, depth-dependent estimate of Manning's coefficient, to yield

$$
\tilde{n}=n\left(\frac{S_{w}}{S_{0}}\right)^{0.38} .
$$

The notation $\tilde{n}$ is used to distinguish the slope-corrected Manning's coefficient from the traditional estimate $n$. If $\tilde{n}$ replaces $n$ in Manning's formula, equation 93, one obtains the steep-slope factor $\Phi$ of equation 92 , at least in the range of slopes between 0.002 and 0.04 , for which the Jarrett formula is supported by data:

$$
\begin{array}{r}
\Phi=\left\{\left(\frac{s_{w}}{s_{0}}\right)^{0.38}\right\}^{2} \\
\text { for } 0.002<s_{w}<0.04
\end{array}
$$

Extend equation 229 to the full range of slopes by defining $\Phi$ to be constant below and above the range specified in equation 229 , and continuous:

$$
\Phi=s_{f a c}^{2}\left(\frac{s_{\min }^{2}}{s_{0}^{2}}\right)^{s_{e}}
$$


where

$$
\begin{aligned}
& s_{f a c}=1 \text { and } s_{e}=0 \\
& \text { if }\left|s_{w}\right|<0.002 \\
& s_{f a c}=1 \text { and } s_{e}=0.38 \\
& \text { if } 0.002 \leq\left|s_{w}\right|<0.04 \\
& s_{f a c}=\left[\frac{0.04}{0.002}\right]^{0.38} \text { and } s_{e}=0 \\
& s_{\min }=\operatorname{ifax}\left(\left|s_{w}\right|, 0.001\right) \operatorname{sign}\left(s_{w}\right) .
\end{aligned}
$$

In equation 234 , the function max indicates the maximum of the two values in parentheses, and the function sign $=1$ if $S_{w} \geq 0$, and -1 otherwise. $S_{w}$ is replaced by $S_{\min }$ for slopes less than 0.001 to avoid division by zero in partial derivatives to be defined below; note that $S_{\text {min }} \neq S_{w}$ only for the case of equation 231 , in which $S_{e}=0$ anyway.

The dependence of $A S_{f}$ on water slope $S_{w}$ through the factor $\Phi$ can now be calculated:

$$
\frac{\partial\left(A S_{f}\right)}{\partial S_{w}}=2 A S_{f} \frac{S_{e}}{S_{\min }} .
$$

The use of water-surface slope $S_{w}$ rather than friction slope $S_{f}$ for the slope $S$ of the steep-slope factor $\Phi$ avoids undesirable numerical feedback between the steep-slope factor and the friction slope that depends on $\Phi$. (See equation 93.) Indeed, to further minimize numerical noise arising from fluctuations in $\Phi$ during the iterations, it is helpful to use only an average value of water slope $S_{w}$ for each space-time finite element. The water surface slope at any point in the space-time element is given by

$$
\frac{\partial z}{\partial x}=\frac{\partial z_{b}}{\partial x}+\frac{\partial h}{\partial x},
$$

where the first term on the right side is given by equation 212 and the second term by equation 102 . The first term is defined by input channel geometry and does not depend on nodal values; the second term depends on nodal values $a_{k}^{h}$ of water depth $h$ as given in equation 103. The average slope $S_{w}$ is taken to be the slope at $(\xi, \eta)=(0,-1)$, that is, in the center of the element in the $x$-direction, and at the old time. Thus, by equation 103 ,

$$
\frac{\partial S_{w}}{\partial a_{k}^{h}}=\left.\frac{\partial N_{k}^{h}}{\partial x}\right|_{\begin{array}{l}
(x(\xi, \eta), t(\xi, \eta))= \\
(x(0,-1), t(0,-1))
\end{array}}
$$

By equations $34,40,41$, and 43 , the right side of the equation is zero for $k=3,4$, and 6 ; that is, there is no dependence on the nodal values at the new time. This nondependence might have been anticipated because $S_{w}$ is based on quantities at $\eta=-1$, that is, on quantities already computed, and should not be affected by changes in nodal values at the new time level. However, the general expression, equation 237, is included for completeness in case one wishes to use other evaluation points besides $(\xi, \eta)=(0,-1)$.

Also, because the average slope $S_{w}$ is evaluated at fixed location $(x(\xi, \eta), t(\xi, \eta))=(x(0,-1), t(0,-1))$ within the element, there is no dependence on $x$-variation of the characteristic path; that is,

$$
\frac{\partial S_{w}}{\partial x}=0
$$

\section{MODEL DEMONSTRATIONS AND VERIFICATIONS}

\section{Example 1}

The use of the model was demonstrated by using a test river channel whose thalweg profile is given in figure 5 and table 1. The profile steepness was mild-steep-mild, becoming as steep as 13 percent at the middle of the reach at river coordinate 25,000 feet. Variable cross-sectional geometry was specified at 2,000 -foot intervals, as given in figure 6 and table 1 . The upstream boundary condition was a specified discharge hydrograph (fig. 7). Downstream from the demonstration reach ( 0 to 50,000 feet), the profile was extended at a slope of 0.1 percent to 60,000 feet, to avoid any lower boundary effect in the example reach.

Model space-time elements were assigned a space dimension equal to 2,000 feet, so that corner nodes coincided with the data cross sections. The downstream boundary condition required that the water depth be the same at the last computational node $(60,000$ feet) and the midside node of the last element $(59,000$ feet $)$. A preliminary model run established a steady-state condition at a discharge of 180 cubic feet per second, corresponding to reference time zero. The upstream input hydrograph (fig. 7) 

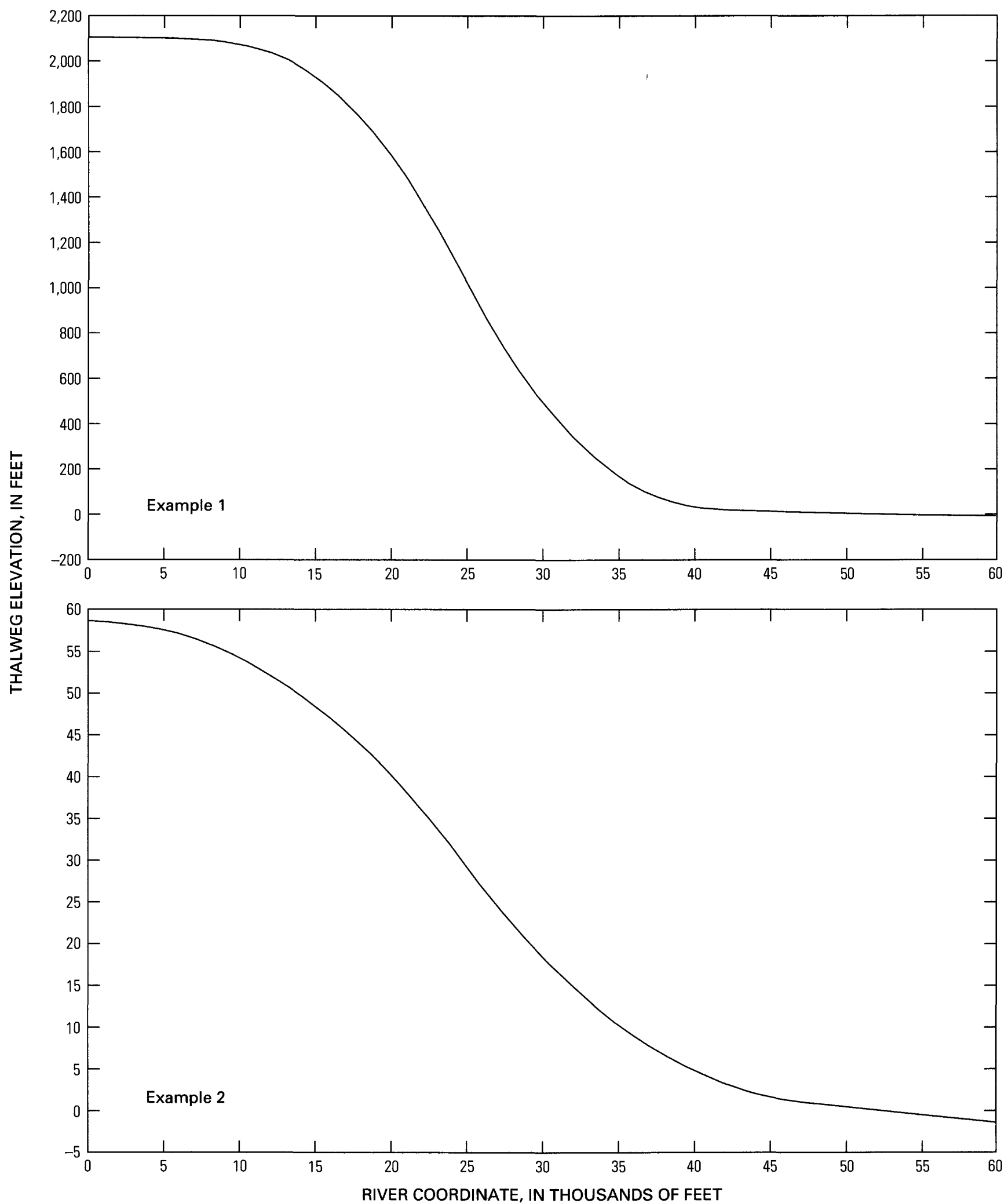

Figure 5. Thalweg profiles for the examples. Note the elevation scale change between the graphs.

then changed abruptly from 180 cubic feet per second at time zero to 1,800 cubic feet per second at time 32 seconds, and remained constant at 1,800 cubic feet per second thereafter. The time increment $\Delta t$ was 4 seconds from time 0 to 120 minutes. A longer time increment of 24 seconds was used between 120 minutes and 8 hours in running the 
Table 1. Cross section geometry of the test river channel.

[See also figures 5 and 6]

\begin{tabular}{|c|c|c|c|c|c|c|c|c|c|c|}
\hline \multirow{2}{*}{$\begin{array}{l}\text { River } \\
\text { coor- } \\
\text { dinate } \\
\text { (feet) }\end{array}$} & \multirow{2}{*}{$\begin{array}{c}\text { Example } 1 \\
\text { thalweg } \\
\text { elevation } \\
\text { (feet) }\end{array}$} & \multirow{2}{*}{$\begin{array}{c}\text { Example } 2 \\
\text { thalweg } \\
\text { elevation } \\
\text { (feet) }\end{array}$} & \multicolumn{8}{|c|}{ Top width, in feet, for given depth, in feet } \\
\hline & & & 0 & 1 & 2 & 3 & 4 & 5 & 6 & 7 \\
\hline & 2,112 & 58.4 & 58 & 70 & 84 & 93 & 98 & 102 & 107 & 111 \\
\hline 2,000 & 2,110 & 58.0 & 7 & 35 & 58 & 71 & 78 & 85 & 91 & 98 \\
\hline 4,000 & 2,108 & 57.6 & 10 & 35 & 45 & 50 & 60 & 75 & 95 & 111 \\
\hline 6,000 & 2,104 & 56.8 & 17 & 41 & 50 & 59 & 74 & 81 & 90 & 103 \\
\hline 8,000 & 2,096 & 55.6 & 13 & 43 & 55 & 62 & 73 & 88 & 94 & 101 \\
\hline 10,000 & 2,080 & 54.0 & 27 & 44 & 54 & 64 & 73 & 79 & 89 & 105 \\
\hline 12,000 & 2,048 & 52.0 & 22 & 46 & 56 & 65 & 72 & 76 & 85 & 96 \\
\hline 14,000 & 1,984 & 49.6 & 35 & 64 & 72 & 82 & 94 & 102 & 109 & 119 \\
\hline 16,000 & 1,888 & 46.8 & 27 & 51 & 60 & 68 & 77 & 89 & 100 & 112 \\
\hline 18,000 & 1,760 & 43.6 & 22 & 51 & 68 & 75 & 81 & 89 & 98 & 109 \\
\hline 20,000 & 1,600 & 40.0 & 23 & 35 & 53 & 62 & 71 & 84 & 93 & 105 \\
\hline 22,000 & 1,408 & 36.0 & 23 & 41 & 54 & 74 & 82 & 103 & 111 & 122 \\
\hline 24,000 & 1,184 & 31.6 & 16 & 41 & 48 & 55 & 63 & 70 & 85 & 113 \\
\hline 26,000 & 928 & 26.8 & 21 & 37 & 53 & 60 & 72 & 87 & 100 & 120 \\
\hline 28,000 & 704 & 22.4 & 21 & 39 & 43 & 52 & 70 & 88 & 99 & 107 \\
\hline 30,000 & 512 & 18.4 & 28 & 45 & 53 & 57 & 60 & 72 & 93 & 121 \\
\hline 32,000 & 352 & 14.8 & 27 & 50 & 62 & 70 & 76 & 90 & 105 & 116 \\
\hline 34,000 & 224 & 11.6 & 20 & 41 & 55 & 64 & 72 & 89 & 96 & 99 \\
\hline 36,000 & 128 & 8.8 & 16 & 46 & 52 & 61 & 71 & 80 & 96 & 112 \\
\hline 38,000 & 64 & 6.4 & 15 & 38 & 56 & 61 & 69 & 95 & 108 & 121 \\
\hline 40,000 & 32 & 4.4 & 25 & 37 & 51 & 58 & 72 & 86 & 91 & 96 \\
\hline 42,000 & 16 & 2.8 & 20 & 36 & 46 & 55 & 69 & 84 & 90 & 104 \\
\hline 44,000 & 8 & 1.6 & 19 & 39 & 58 & 68 & 79 & 83 & 92 & 104 \\
\hline 46,000 & 4 & 0.8 & 22 & 40 & 50 & 66 & 75 & 81 & 92 & 105 \\
\hline 48,000 & 2 & 0.4 & 15 & 36 & 49 & 60 & 72 & 81 & 91 & 104 \\
\hline 50,000 & 0 & 0.0 & 15 & 42 & 53 & 66 & 80 & 89 & 98 & 110 \\
\hline 52,000 & -2 & -0.4 & 15 & 42 & 53 & 66 & 80 & 89 & 98 & 110 \\
\hline 54,000 & -4 & -0.8 & 15 & 42 & 53 & 66 & 80 & 89 & 98 & 110 \\
\hline 56,000 & -6 & -1.2 & 15 & 42 & 53 & 66 & 80 & 89 & 98 & 110 \\
\hline 58,000 & -8 & -1.6 & 15 & 42 & 53 & 66 & 80 & 89 & 98 & 110 \\
\hline 60,000 & -10 & -2.0 & 15 & 42 & 53 & 66 & 80 & 89 & 98 & 110 \\
\hline
\end{tabular}

model to a steady-state condition at a discharge of 1,800 cubic feet per second. A Manning's " $n$ " of 0.030 was used throughout. The neighborhoods of restriction were specified by setting $\chi=0.5$ and $\theta=0.5$. The model's steep-slope factor was set to unity for this example, to allow comparison with another model and to demonstrate transitions between supercritical and subcritical flow. The flow changed from subcritical to supercritical near river distance 10,000 feet, and back from supercritical to subcritical near 40,000 feet. The depth profiles (fig. 8) show the flood wave progressing down the river channel.
The steady-state conditions at the start and end of modeling were compared with results from the U.S. Geological Survey's step-backwater program J635 (Shearman, 1976; written commun., 1977). River depths obtained using J635 are plotted in figure 8 as squares, and show excellent agreement with depth-profile curves of the finiteelement model. (The J635 model can only treat the steadystate case.) Mass conservation was demonstrated by finding the difference between inflow at the upstream end of the reach and outflow at the downstream end, and comparing that figure to the change in channel storage within the reach. 


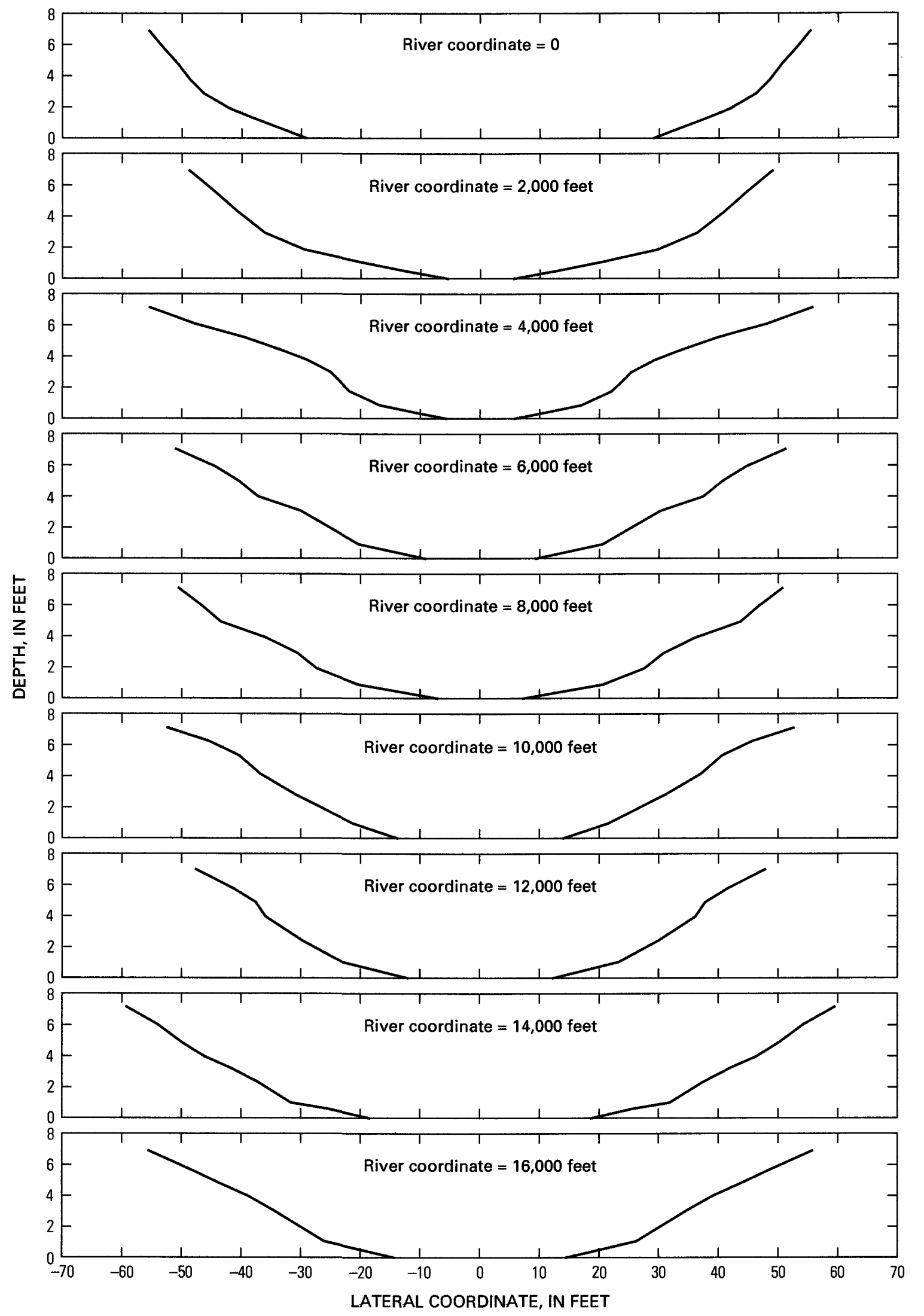

Figure 6 (above and following two pages). Data cross sections of the test river channel, at 2,000-foot intervals, for both examples 1 and 2 . 


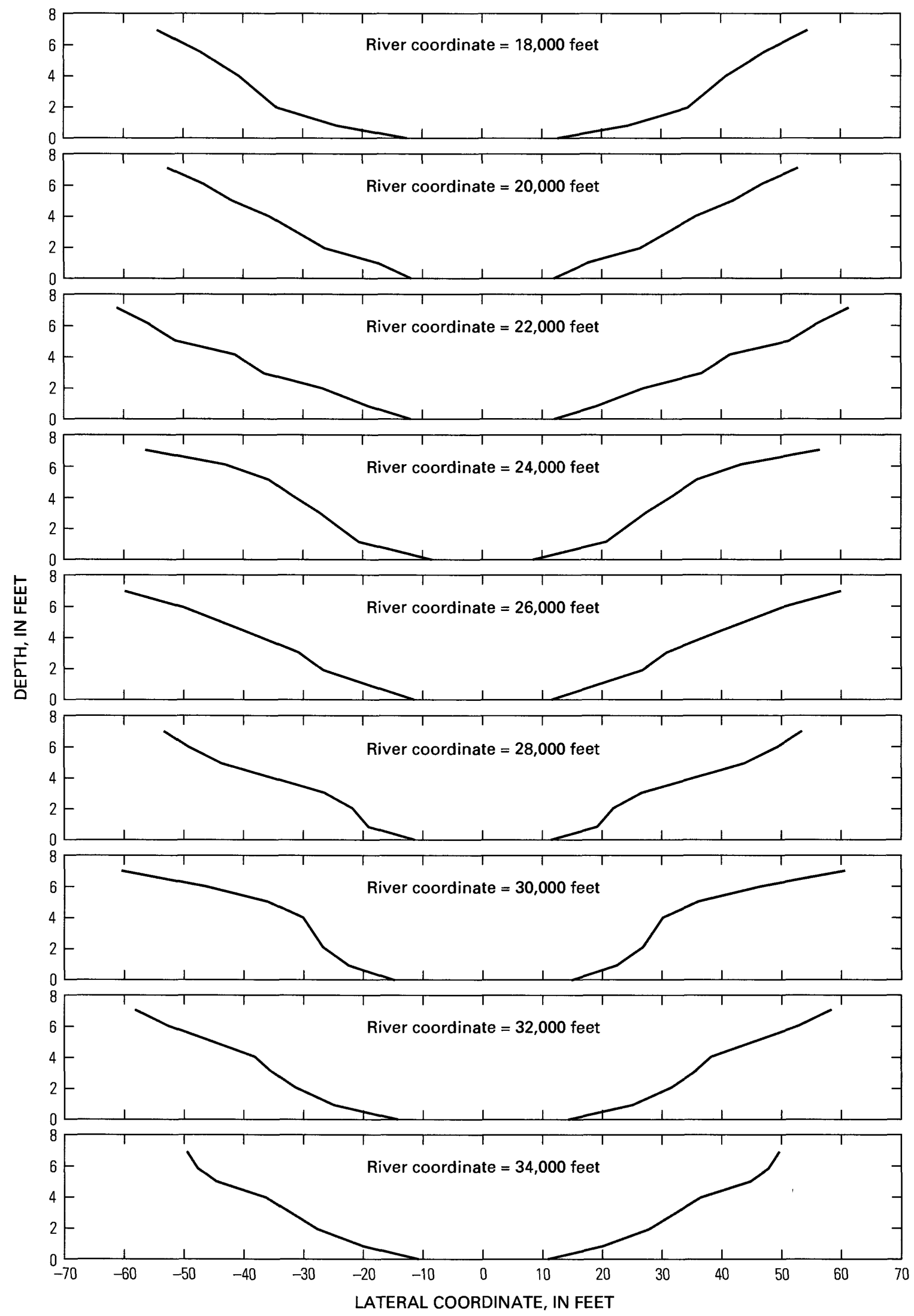

Figure 6. Data cross sections of the test river channel-Continued. 


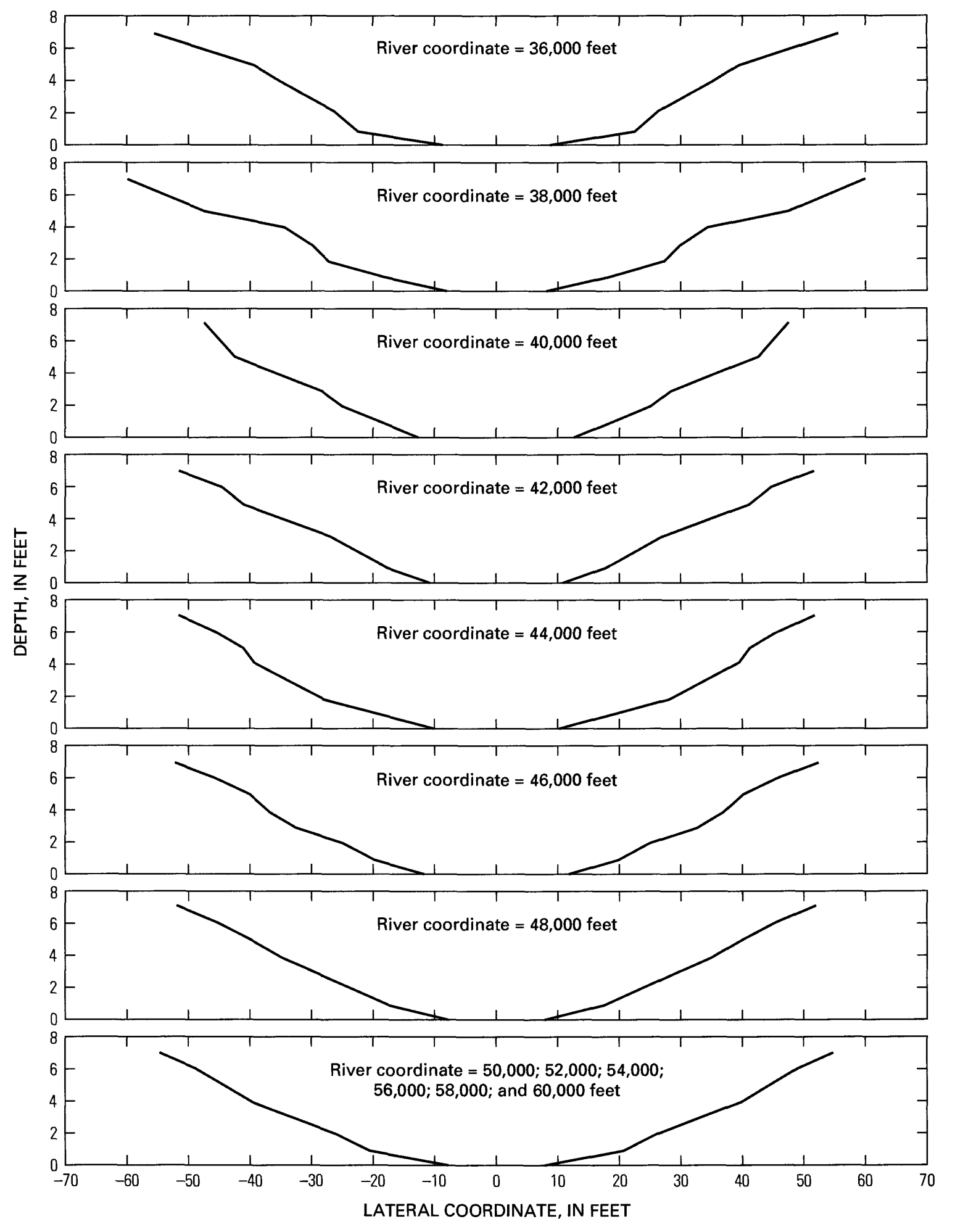

Figure 6. Data cross sections of the test river channel-Continued. 

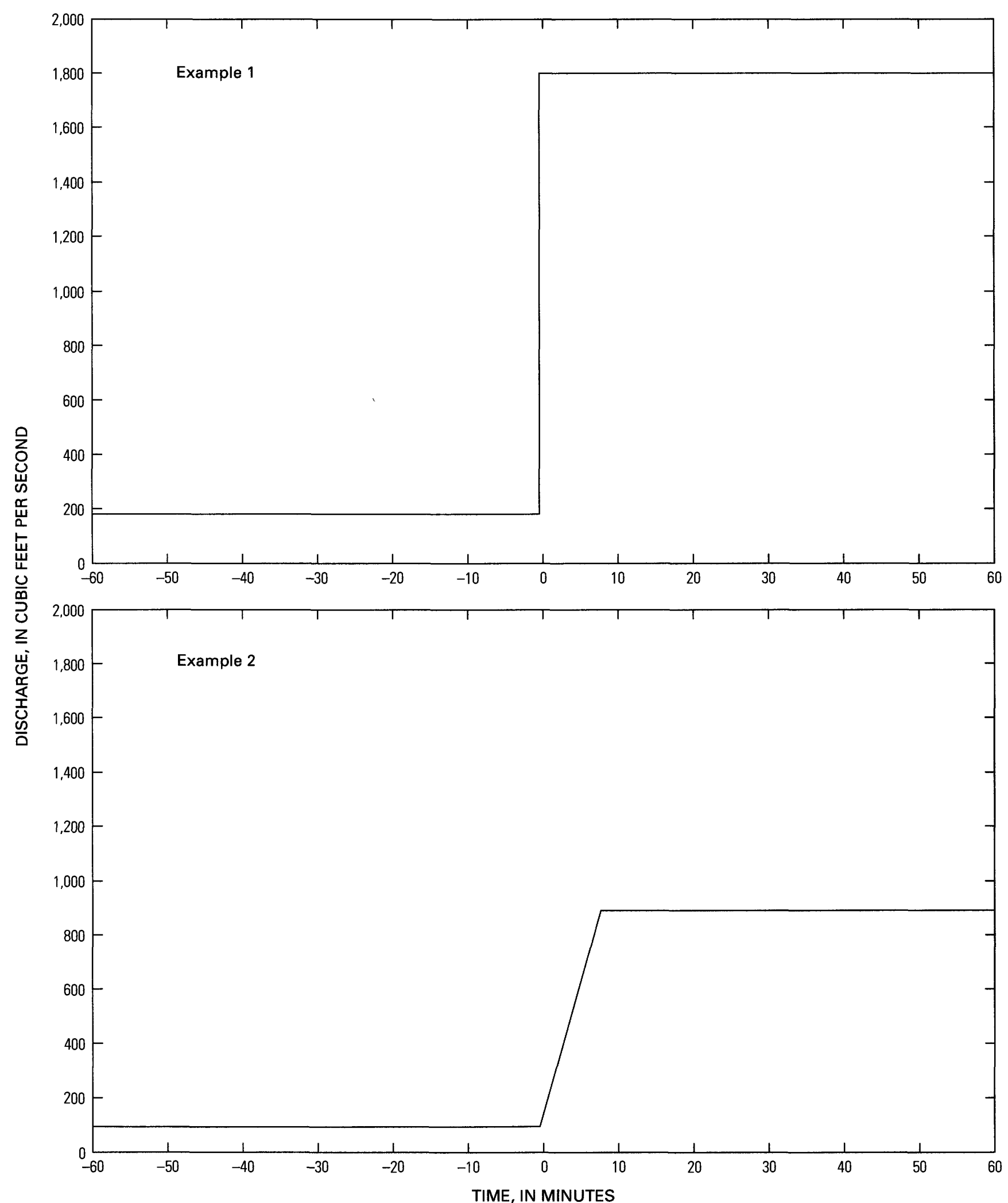

Figure 7. Input discharge hydrographs for the examples. The discharge hydrographs continue at the indicated constant step values during times before and after those shown in these graphs. 
The time interval 0 to 60 minutes, during the most dynamic part of the flood-wave passage, was chosen for the comparison:

\begin{tabular}{|c|c|}
\hline & Cubic feet \\
\hline Inflow & $\begin{array}{l}64,540,800 \\
-6,480,149\end{array}$ \\
\hline 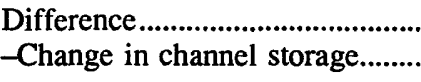 & $\begin{array}{r}58,060,651 \\
-58,131,844\end{array}$ \\
\hline Error & $-71,193$ \\
\hline
\end{tabular}

The error represents only 0.1 percent of the inflow to the reach. In this mass conservation check, the volume in channel storage was calculated using two-point Gaussian integration, over the length $\Delta x$ of each computational element, of cross-sectional area below the water surface. Inflowing and outflowing volumes were calculated by trapezoidal-rule time integration of the inflowing and outflowing discharge hydrographs.

Another indication of mass conservation was provided by the observation that for steady-state discharge, the modeled discharges through all cross sections were the same. For example, the modeled discharge at 8 hours was computed as $1,800.000000$ cubic feet per second at every cross section. This seemingly simple requirement of constant discharge throughout the reach in steady state is one that standard implementations of the implicit method of characteristics will not satisfy unless the time steps are large enough that the characteristics intercept the sides of the space-time elements, rather than the bases (fig. 1). For highly dynamic problems, such large time steps are not practical.

\section{Example 2}

The model also was compared with dynamic model DWOPER (Fread, 1978). DWOPER is based on the fourpoint implicit computational scheme. Experience has shown that the DWOPER algorithm works satisfactorily for gently sloping streams and slowly changing dynamic conditions, but is more difficult to use on steep slopes and (or) under rapidly changing flow conditions. To facilitate comparison of the new model with DWOPER, example 2 was based on the same cross sections used in example 1 but had a much gentler thalweg profile (figs. 5 and 6 ; table 1). The profile included gentle slopes of 0.02 percent near the ends, steepening to only 0.24 percent near the middle of the profile at 25,000 feet; this mild slope ensured that the flow was always subcritical. The input discharge hydrograph at the upstream boundary was similar to that of example 1 , but rose less abruptly from a low constant discharge of 90 cubic feet per second at time zero to a high constant discharge of 900 cubic feet per second at time 8 minutes (fig. 7). The water depth at the downstream boundary $(60,000$ feet) was set at 5 feet to provide the second required boundary condition. The computational elements had space dimen- sions of 2,000 feet, as in example 1, and again the corner nodes coincided with the data cross sections. The DWOPER computational nodes were also chosen at the 2,000-foot spacing of the data cross sections. A time step of 1 minute was used for both models. A Manning's coefficient of 0.030 was used throughout, and the steep-slope factor was again set at unity. The neighborhoods of restriction were specified by setting the parameters $\chi=0.5$ and $\theta=0.5$.

Results of the comparison are given in the depth profiles of figure 9. Only that part of the reach from 0 to 50,000 feet was compared in order to stay some distance from the lower boundary; the backwater curve from the 5-foot downstream depth is, however, still visible near 50,000 feet because of the gentle slope. The solid curves in figure 9 were computed using the model described in this paper. The overlying squares show depths computed using DWOPER. Overall agreement between the two models was excellent, except near the flood-profile front, where DWOPER water depths were too small. This phenomenon is a known difficulty of the DWOPER 4-point scheme that is caused by high-frequency waves numerically moving ahead of the main flood rise.

A mass conservation check again showed excellent balance. From time zero to 4 hours, over the reach from 0 to 50,000 feet, the new model computed the following volumes:

\begin{tabular}{|c|c|}
\hline & Cubic feet \\
\hline Inflow & $\begin{array}{r}12,765,600 \\
-2,403,424\end{array}$ \\
\hline 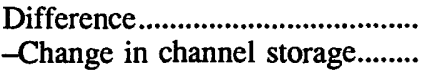 & $\begin{array}{r}10,362,176 \\
-10,371,201\end{array}$ \\
\hline Error & $-9,025$ \\
\hline
\end{tabular}

This error represents only 0.07 percent of the inflow volume to the reach. It was not necessary to run this example as long as example 1 , because comparison in example 2 is with a dynamic model, and there was no need to reach steady state, as was the case in example 1. The model's approximation to mass conservation seems to be slightly better with milder slopes, more nearly prismatic channel geometry, and less sharply rising hydrographs, given the same cross-sectional spacing.

The effect of using restricted neighborhoods can be shown using the data of example 2 . In the first demonstration, the water-depth profile of example 2, as run above using $\chi=0.5, \theta=0.5$, and $\Delta t=4$ seconds, was compared against results of a model run using neighborhoods equal to the full space-time elements, that is, using $\chi=1$ and $\theta=1$ (fig. 10). Because of the relatively short time steps, specifying $\chi=0.5$ and $\theta=0.5$ was equivalent to specifying $\chi=1.0$ and $\theta=0.5$; thus, the comparison focuses on restricting the neighborhood vertically. Figure 10 shows that the restricted neighborhoods diminish overshoot of the profile below the initial steady-state profile at the front, at the expense of some spreading of the front. 
In a second demonstration, example 2 was rerun using $\Delta t=15$ minutes, and the water-surface profile obtained by using 0.5 for both $\chi$ and $\theta$ was compared with that obtained using 1 for both parameters (fig. 11). Because of the relatively long time steps, choosing $\chi=0.5$ and $\theta=0.5$ was equivalent to specifying $\chi=0.5$ and $\theta=1$; therefore, this comparison focuses on restricting the neighborhood horizontally. Figure 11 shows that the restricted neighborhoods prevent oscillations in the profile.

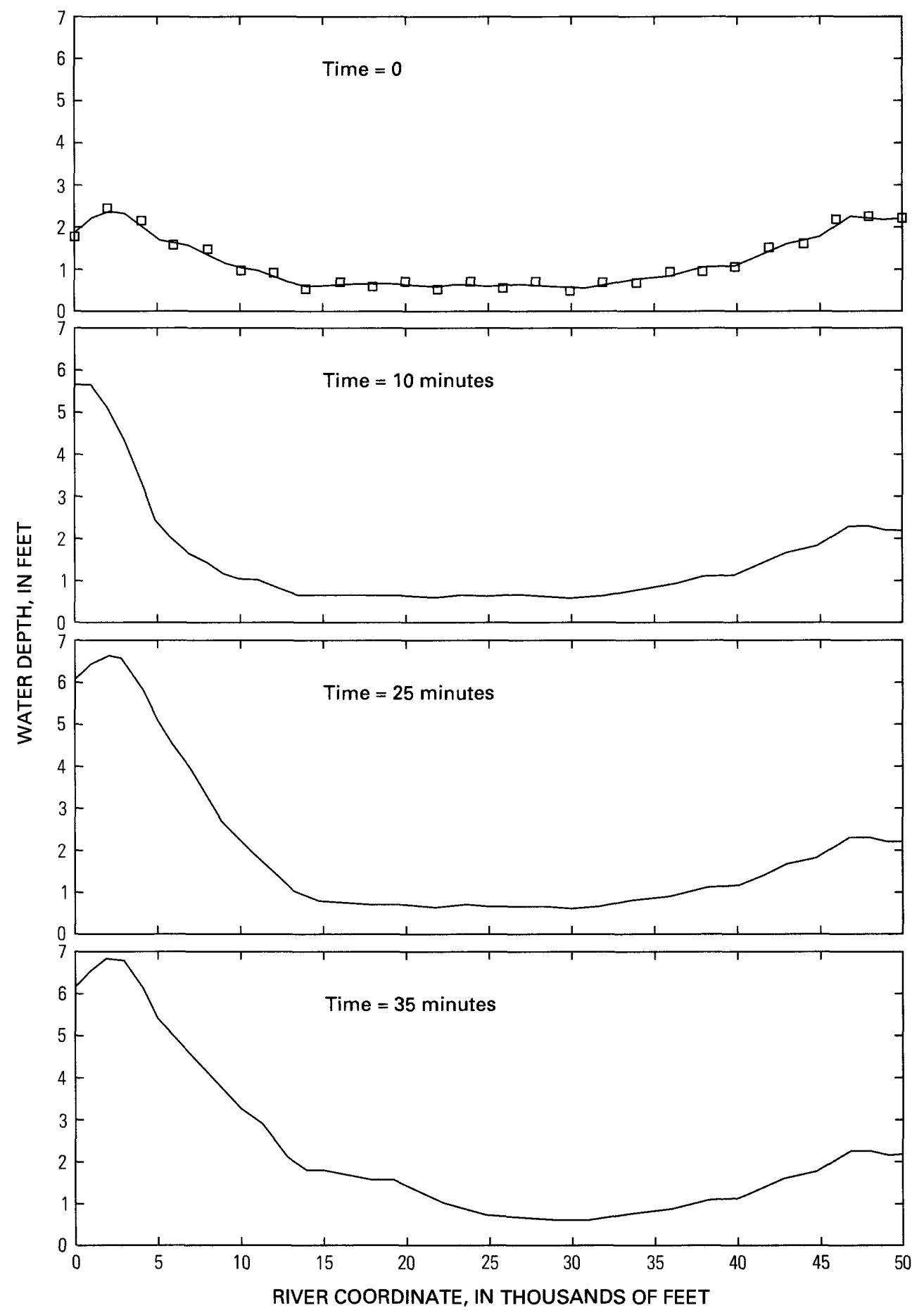

Figure 8 (above and facing page). Computed water-depth profiles for example 1. For comparison, the squares on the steady-state profiles at time zero and at time $=8$ hours indicate depths calculated by step-backwater program $J 635$ for a discharge of 180 cubic feet per second. 


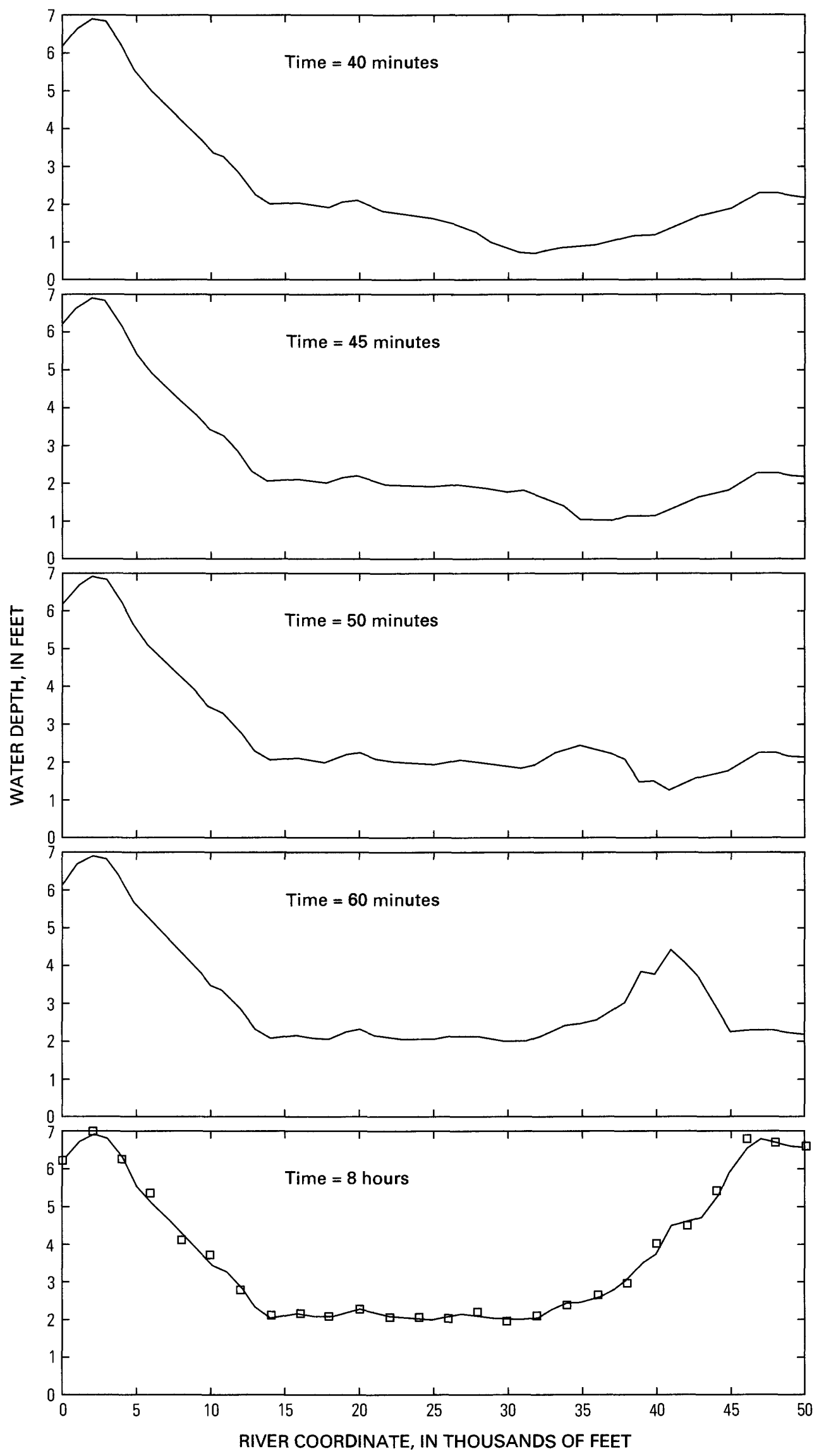




\section{FUTURE EXTENSION}

The model described in this paper could form a basis for a one-dimensional sediment transport model that is suitable for rivers on steep slopes. In addition, future investigations may show whether the approach can be extended to multidimensional streamflow models for rivers on steep slopes.

\section{SUMMARY AND CONCLUSIONS}

A robust computer model that can be used for unsteady streamflow on steep slopes has been presented.

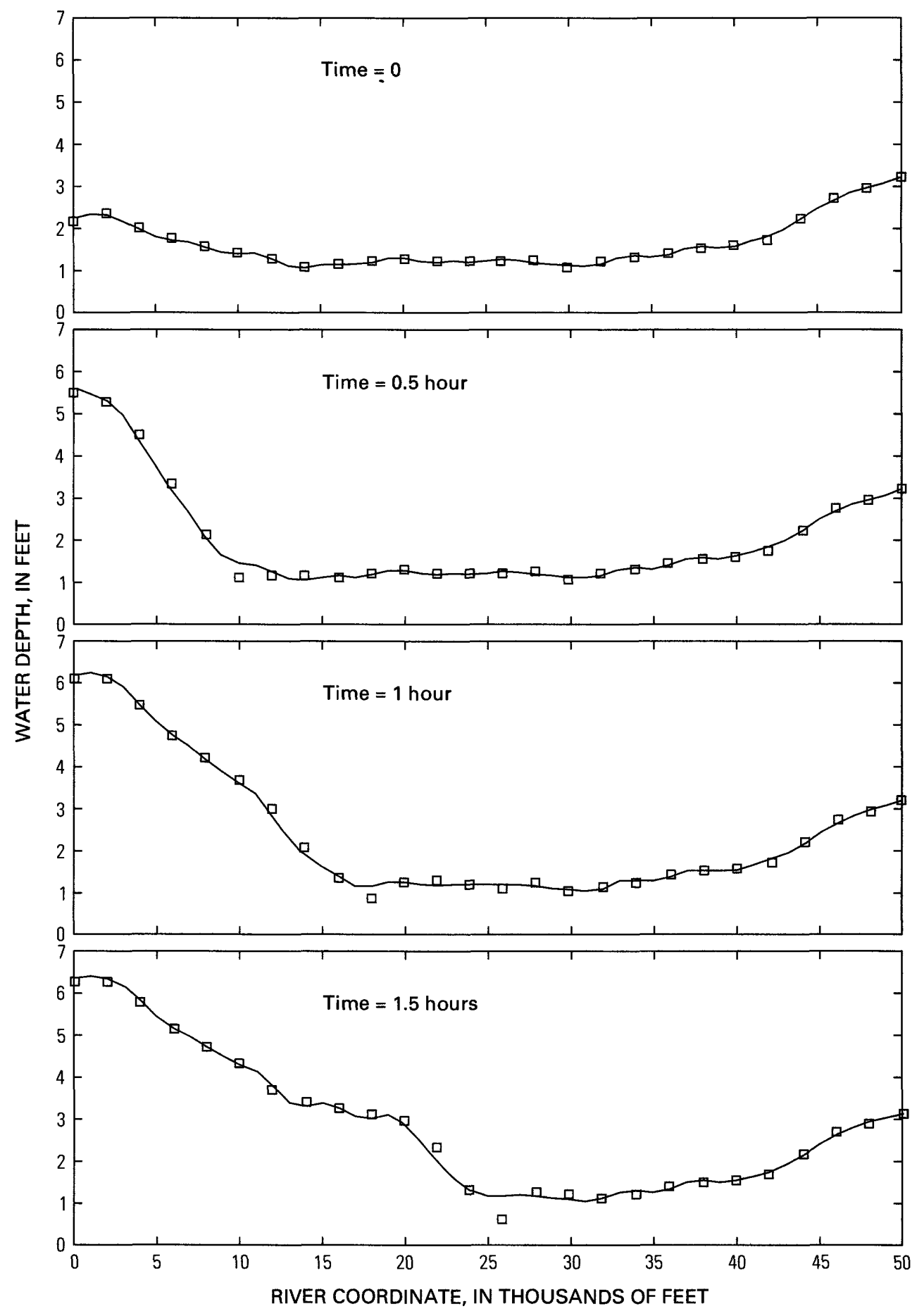

Figure 9 (above and facing page). Computed water-depth profiles for example 2. For comparison, the squares indicate depths calculated by the DWOPER computer model. 

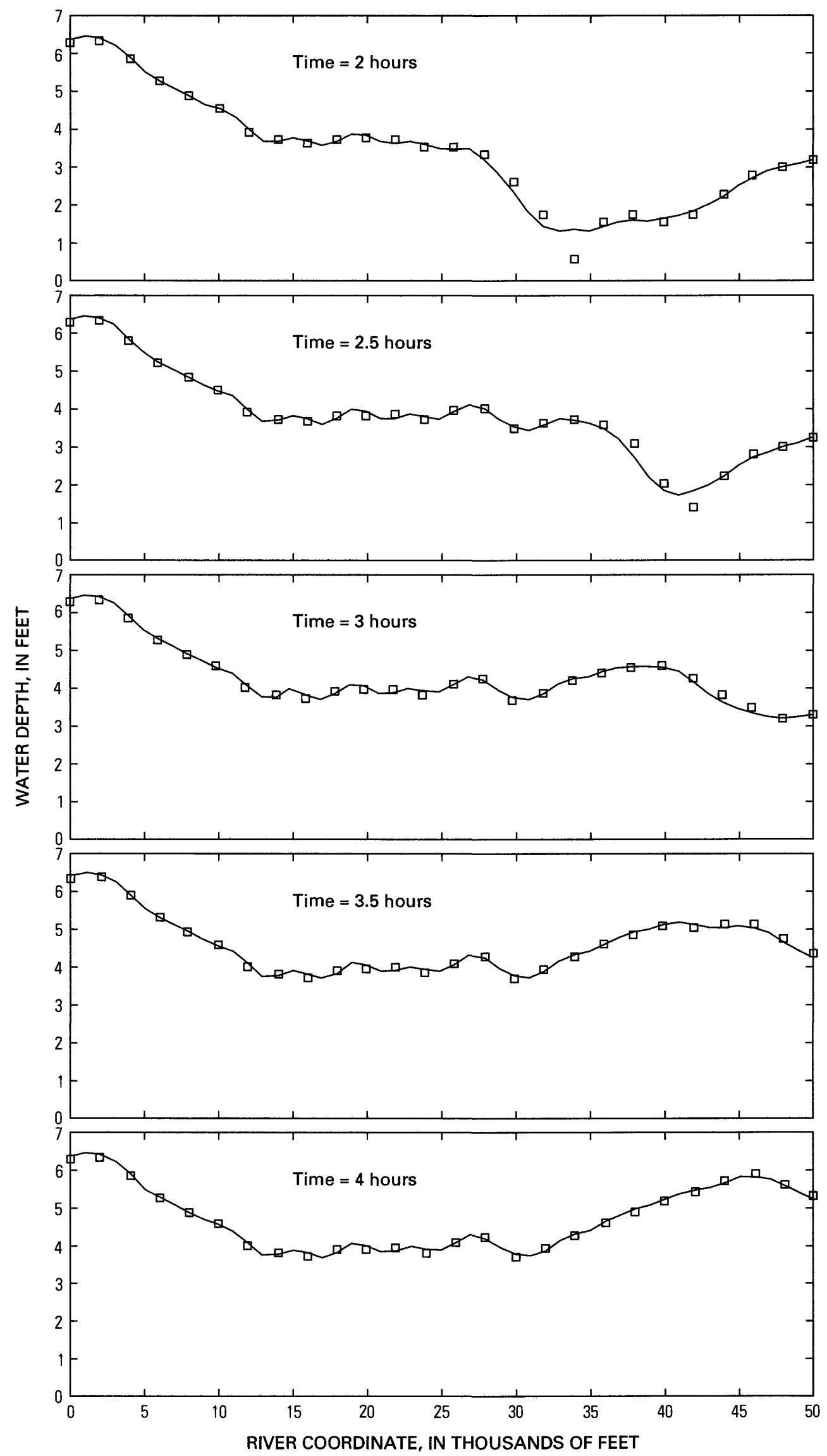


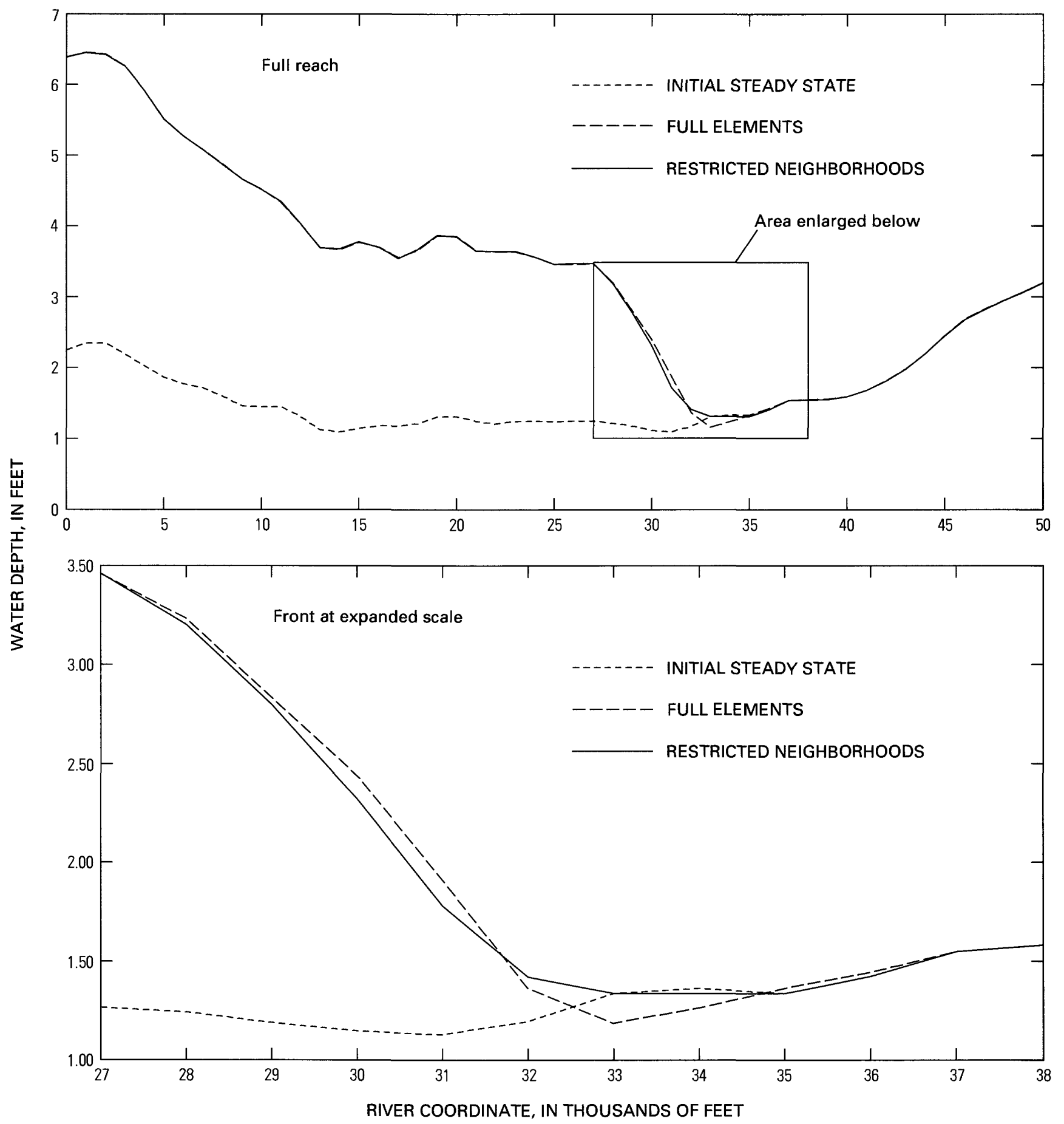

Figure 10. Computed water-depth profiles showing effect of restriction of neighborhoods in time. Data of example 2 were used. At time $=2$ hours, the profile computed using restricted neighborhoods specified by $\chi=0.5$ and $\theta=0.5$ is compared with the profile computed with full-element neighborhoods having $\chi=1$ and $\theta=1$. The time dimension of the neighborhoods is restrictive because of the relatively short time step of $\Delta t=4$ seconds. The initial steady-state depth profile is shown for reference.

The basis for the model is the method of characteristics on a computational grid for which space-time nodes are specified independent of the characteristic paths. The model uses a finite element setting. Because standard implementations of the method of characteristics fail to conserve mass, an additional continuity equation requirement has been added on each space-time element to ensure mass conservation. Channel parameters are approximated by Hermite interpolation to ensure enough smoothness with respect to river coordinate $x$ and depth $h$; the smooth interpretation avoids numeric difficulties that occur on steep slopes because of discontinuities in parameters such as the 


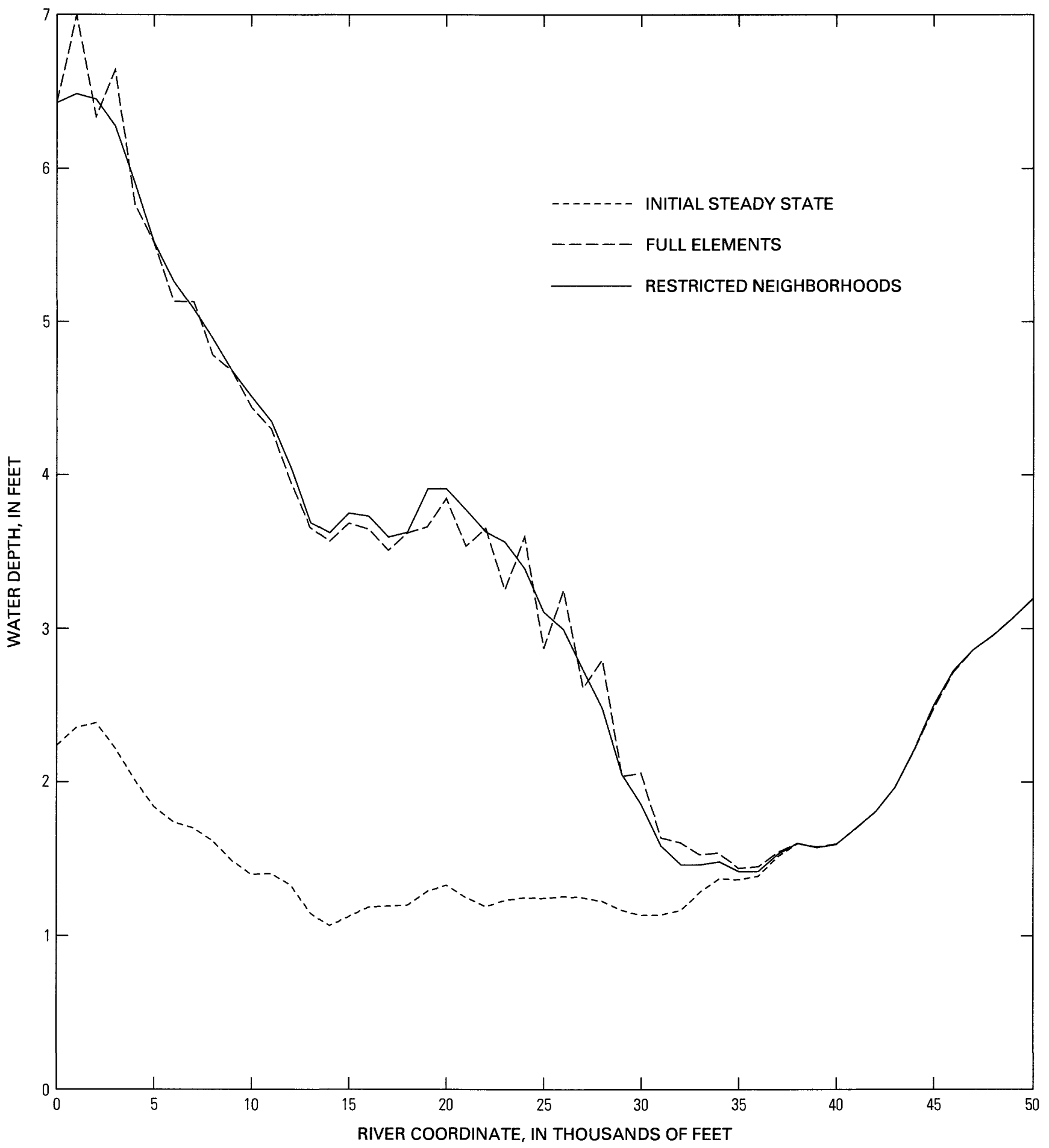

Figure 11. Computed water-depth profiles showing effect of restriction of neighborhoods in space. Data of example 2 were used, except that $\Delta t=15$ minutes. At time $=2$ hours, the profile computed using restricted neighborhoods specified by $\chi=0.5$ and $\theta=0.5$ is compared with the profile computed with full-element neighborhoods having $\chi=1$ and $\theta=1$. The space dimension of the neighborhoods is restrictive because of the relatively long time step. The initial steady-state depth profile is shown for reference.

top width $B$. Channel parameters are interpolated from input data cross sections that are independent of computational elements and nodes, allowing concentration of the computational elements in regions that experience large changes in discharge $Q$ and (or) water depth $h$. A procedure has been included for modifying Manning's friction slope to make it appropriate for steep river slopes, although this steep-slope factor is not otherwise needed for proper 
performance of the model. The model is not restricted to steep slopes, but is applicable to gently sloping streams as well.

\section{REFERENCES}

Chow, V.T., 1959, Open-channel hydraulics: New York, McGrawHill Book Company, 680 p.

Cunge, J.A., Holly, F.M., Jr., and Verwey, Adrianus, 1980, Practical aspects of computational river hydraulics: London, Pitman Publishing Ltd., 420 p.

Edenhofer, Johann, and Schmitz, Gerd, 1981, Ein implizites Charakteristikenverfahren zur Lösung von Anfangsrandwertaufgaben bei hyperbolischen Systemen und seine Konvergenz: Computing, v. 26, p. 257-264.

Franz, D.D., 1976, Discussion of paper 11910, Dimensionless solution of dam-break flood waves, by John G. Sakkas and Theodor Strelkoff: Journal of the Hydraulics Division, American Society of Civil Engineers, v. 102, no. HY12, p. 1782-1784.

Fread, D.L., 1978, NWS operational dynamic wave model, in Verification of Mathematical and Physical Models in Hydraulic Engineering: American Society of Civil Engineers, Hydraulics Division, 26th Annual Specialty Conference, College Park, Maryland, August 9-11, 1978, Proceedings, p. 455-464.
Glass, W.L., Milhous, R.T., Riggs, H.C., Thompson, S.M., and Jarrett, R.D., 1987, Discussion and closure of paper 19272, Hydraulics of high-gradient streams, by Robert D. Jarrett: Journal of Hydraulic Engineering, American Society of Civil Engineers, v. 113, no. 7, p. 918-929.

Goldberg, D.E., and Wylie, E.B., 1983, Characteristics method using time-line interpolations: Journal of Hydraulic Engineering, American Society of Civil Engineers, v. 109, no. 5 , paper no. 17937 , p. $670-683$.

Jarrett, R.D., 1984, Hydraulics of high-gradient streams: Journal of Hydraulic Engineering, American Society of Civil Engineers, v. 110, no. 11, paper no. 19272, p. 1519-1539.

Lai, Chintu, 1988, Comprehensive method of characteristics models for flow simulation: Journal of the Hydraulics Division, American Society of Civil Engineers, v. 114, no. 9, paper no. 22726, p. 1074-1097.

Sakkas, J.G., and Strelkoff, Theodor, 1976, Dimensionless solution of dam-break flood waves: Journal of the Hydraulics Division, American Society of Civil Engineers, v. 102, no. HY2, Proceedings Paper 11910, p. 171-183.

Shearman, J.O., 1976, Computer program E431 user's manual: U.S. Geological Survey Open-File Report 76-499, 103 p.

Wylie, E.B., 1980, Inaccuracies in the characteristics method, in Ashton, George, ed., Proceedings of the Specialty Conference on Computer and Physical Modeling, Chicago, Aug. 6-8, 1980: New York, American Society of Civil Engineers, p. 165-176.

Zienkiewicz, O.C., 1977, The finite element method (3d ed.): London, McGraw-Hill, 787 p. 


\section{INDEX OF VARIABLE DEFINITIONS, INTERPOLATIONS, AND DERIVATIVES}

The associated equation number is shown. In some cases, the equation referenced is written in terms of another variable, but a similar equation applies to the indexed variable; in these cases, the equation number is enclosed in parentheses.

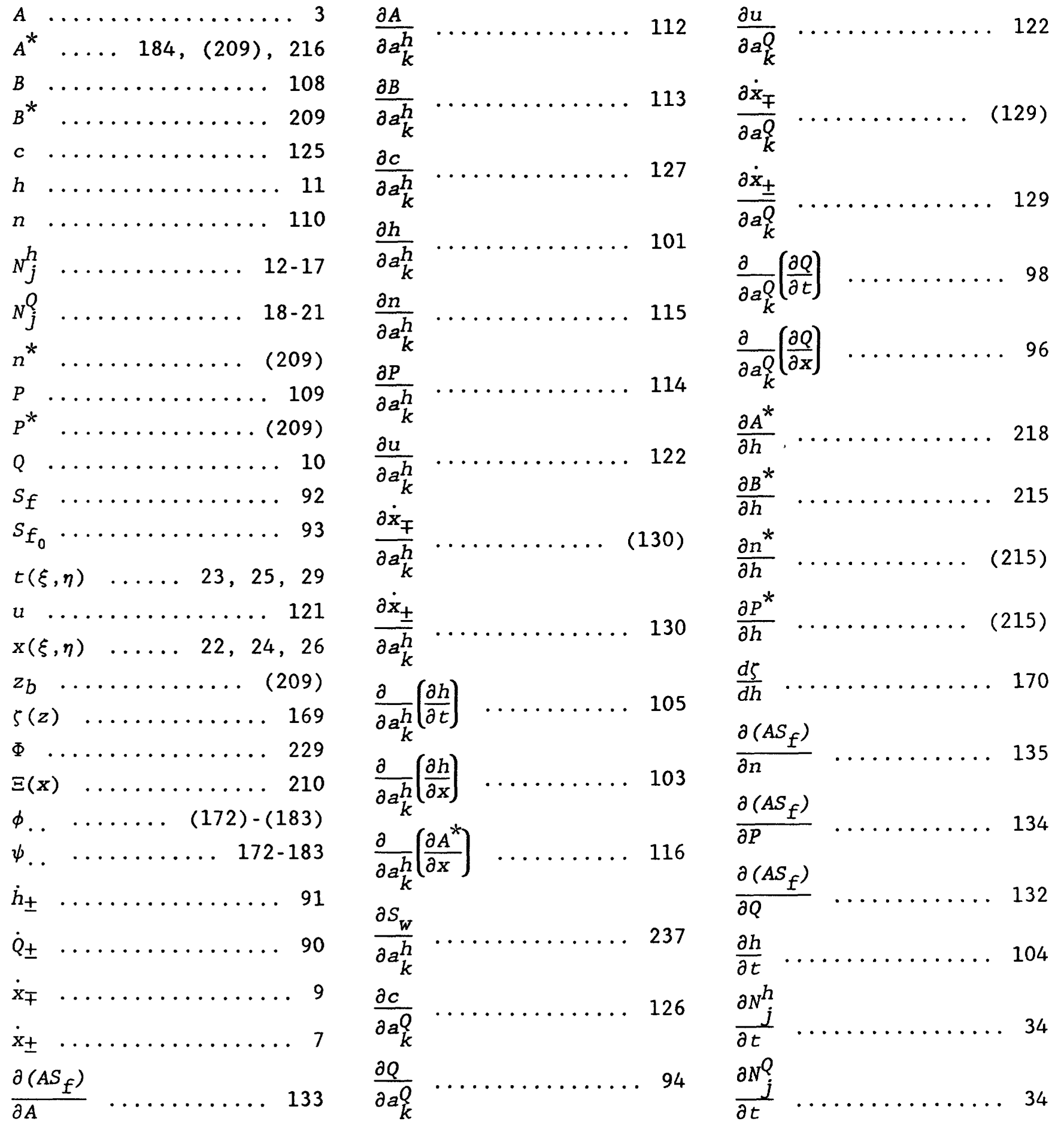




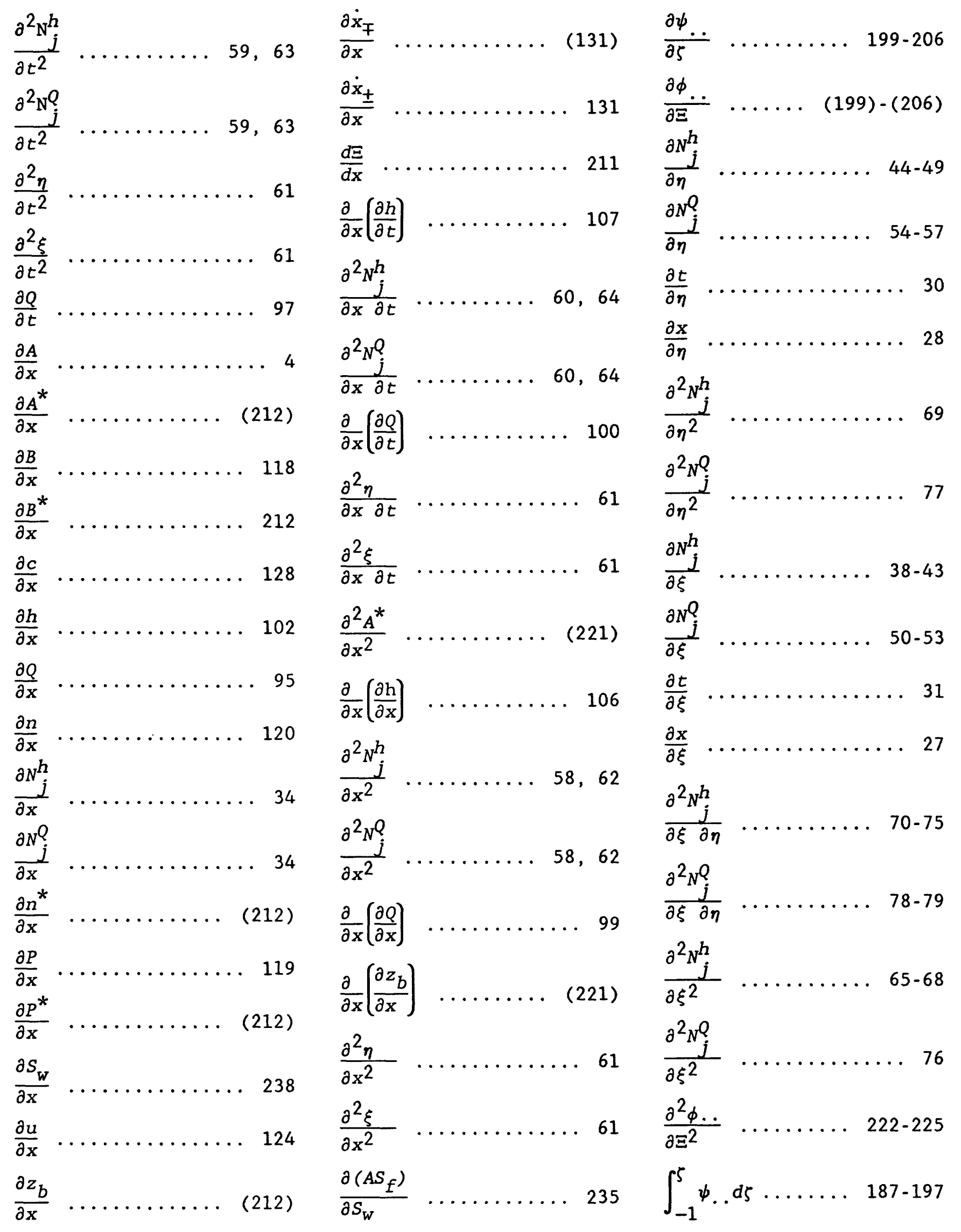

44 Mass-Conserving Method of Characteristics for Streamflow Modeling 